\title{
1,3-Oxyalkynylation of aryl cyclopropanes with ethylnylbenziodoxolones using photoredox catalysis
}

\author{
Zhijun Zuo and Armido Studer*
}

Institute of Organic Chemistry, University of Münster, Corrensstrasse 40, 48149 Münster, Germany

Table of Contents:
A. General information:.
B. Preparation of substrates:
C. Catalytic results: S7
D. Large-scale preparation of $3 \mathrm{a}$ and its derivatization:. . $\mathrm{S} 25$
E. Mechanistic studies: S28
G. References: .529
H. NMR spectrum: S30 


\section{A. General information:}

All reactions involving air- or moisture-sensitive reagents or intermediates were carried out in pre-heated glassware using standard Schlenk techniques. All commercially available reagents were purchased from Sigma-Aldrich, Alfa Aesar, TCI Chemicals, Acros Organics or ABCR in the highest purity grade and used without further purification. DCE and $\mathrm{CHCl}_{3}(99,8 \%$, extra dry, AcroSeal) was used as received from Acros Organics.

Thin layer chromatography (TLC) was performed on Merck silica gel 60 F-254 plates and visualized by fluorescence quenching under UV light.

Column chromatography was performed on Merck or Fluka silica gel 60 (40-63 $\mu \mathrm{m})$.

${ }^{1} \mathrm{H}$ NMR and ${ }^{13} \mathrm{C}$ NMR spectra were recorded on a Bruker DPX 300, a Bruker AV 400 at $300 \mathrm{~K}$, a Varian INOVA 500 or a Varian 600 UNITY plus spectrometer at 299 K. Spectra were calibrated relative to solvent's residual proton and carbon chemical shift: $\mathrm{CHCl}_{3}\left(\delta=7.27 \mathrm{ppm}\right.$ for ${ }^{1} \mathrm{H}$ NMR and $\delta=77.0 \mathrm{ppm}$ for ${ }^{13} \mathrm{C}$ NMR). Data are reported as follows: chemical shift, multiplicity $(\mathrm{s}=$ singlet, $\mathrm{d}=\mathrm{doublet}, \mathrm{t}=$ triplet, $\mathrm{q}=$ quartet, brs = broad singlet, $\mathrm{m}=$ multiplet $)$, coupling constants $(\mathrm{Hz})$ and integration.

HRMS ESI (m/z) spectra were recorded on a Bruker MicroTof or an Orbitrap LTQ XL (Nanospray) of Thermo Scientific. 


\section{B. Preparation of substrates:}

Substrates Preparation:

aryl cyclopropanes:
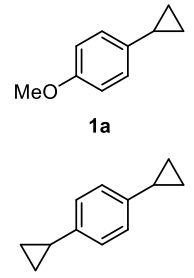

1d

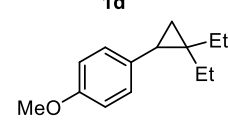

$1 \mathrm{~g}$<smiles>COc1ccc(C2CC2(C)C)cc1C(C)=O</smiles><smiles>CC(C)Cc1ccc(C2CC2(C)C)cc1</smiles>

$1 \mathrm{~m}$

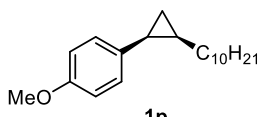

$1 \mathrm{p}$<smiles>COc1ccc(C2CC2Cc2ccccc2)cc1</smiles>

$1 \mathrm{~s}$<smiles>c1ccc(C2CC2)cc1</smiles>

$1 b$<smiles>COc1ccccc1C1CC1</smiles>

$1 \mathrm{e}$<smiles>COc1ccc(C2CC2(C)C)cc1Cl</smiles><smiles>COc1ccc(C2CC2(C)C)cc1C(F)(F)F</smiles>

$1 \mathrm{k}$

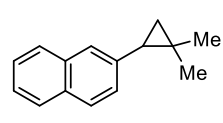

$1 \mathrm{n}$

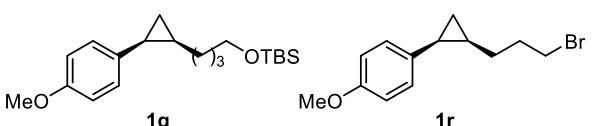

$1 q$

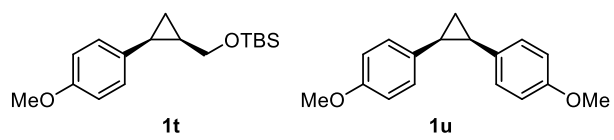

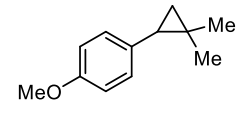

$\overbrace{\mathrm{Me}}^{\mathrm{Br}}$
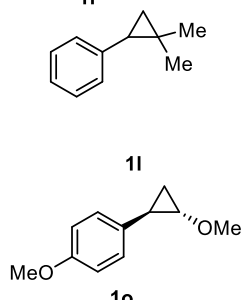

(trans:cis $=0.25: 1$ )

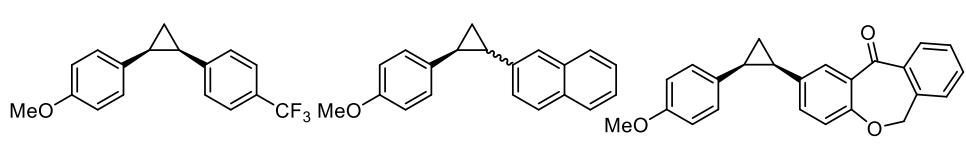

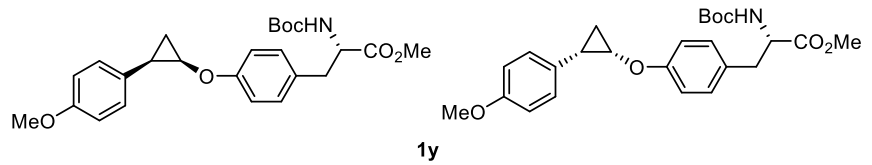

(1)<smiles>COc1ccc([C@H]2C[C@H]2Oc2ccc3c(c2)CC[C@@H]2C3CC[C@]3(C)C(=O)CC[C@@H]23)cc1</smiles> 

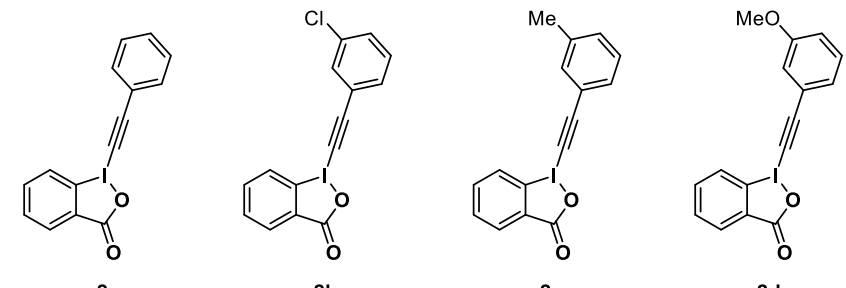

2b
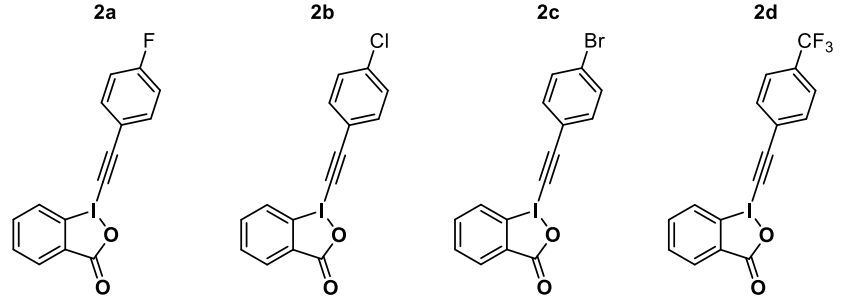

$2 e$

$2 f$

$2 \mathrm{~g}$

$2 \mathrm{~h}$
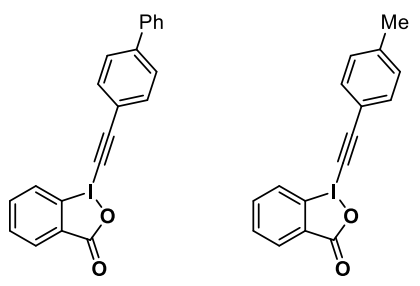

$2 \mathrm{j}$

\section{Preparation of aryl cyclopropanes:}

\section{General procedure $A::^{[1]}$}

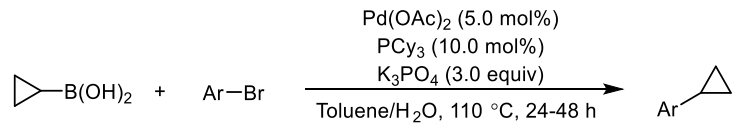

In a $50 \mathrm{~mL}$ Schlenk tube with magnetic stirring bar were added the corresponding aryl bromide (3.0 mmol, 1 equiv), tricyclohexylphosphine $(84.0 \mathrm{mg}, 0.30 \mathrm{~mol}, 0.1$ equiv), palladium acetate $(33.7 \mathrm{mg}, 0.15$ mol, 0.05 equiv), tripotassium phosphate (1.90 g, $9.0 \mathrm{~mol}, 3.0$ equiv) and cyclopropylboronic acid (387 $\mathrm{mg}, 4.5 \mathrm{mmol}, 1.5$ equiv). Then toluene $(0.3 \mathrm{M})$ and $0.5 \mathrm{~mL}$ of water were added, and the tube was closed. The reaction mixture was shaken briefly and set under inert atmosphere by bubbling nitrogen gas through the vial for 5 minutes. Afterwards, the tube was placed into an oil bath and stirred at $110^{\circ} \mathrm{C}$ for $24-48 \mathrm{~h}$. Upon completion, the reaction mixture was poured into a separatory funnel, diluted with ethyl acetate and washed with $15 \mathrm{~mL}$ water twice. The organic layer was dried with $\mathrm{Na}_{2} \mathrm{SO}_{4}$, concentrated in vacuo and purified by column chromatography to give the title compound. Compounds 1a-e were prepared following the General Procedure A.

\section{General procedure B: ${ }^{[2]}$}

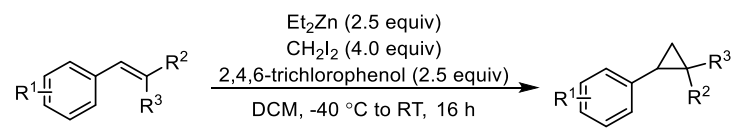


In a $100 \mathrm{~mL}$ oven-dried round-bottom flask with a stir bar, was added 2,4,6-trichlorophenol (1.18 g, 6.0 mmol, 2.5 equiv) under nitrogen atmosphere. DCM $(60 \mathrm{~mL}, 0.1 \mathrm{M})$ was added into the flask and the reaction mixture was cooled to $-40{ }^{\circ} \mathrm{C}$. $\mathrm{ZnEt}_{2}(1.0 \mathrm{M}, 6.0 \mathrm{~mL}, 6.0 \mathrm{mmol}, 2.5$ equiv) was added slowly into the flask by syringe and the reaction mixture was stirred at this temperature for $15 \mathrm{~min} . \mathrm{CH}_{2} \mathrm{I}_{2}(2.57$ g, 9.6 mmol, 4.0 equiv) was added slowly by syringe and the reaction mixture was stirred at this temperature for another $15 \mathrm{~min}$. Next, the corresponding solution of alkene (2.4 mmol, 1.0 equiv) in DCM $(10 \mathrm{~mL})$ was added by syringe and the reaction mixture was allowed to warm to room temperature and stirred for $16 \mathrm{~h}$. After the reaction reached completion, the reaction mixture was quenched with sat. $\mathrm{NH}_{4} \mathrm{Cl}(30 \mathrm{~mL})$ and extracted with DCM $(100 \mathrm{~mL})$ for 3 times. The combined organic layers were washed with aq. $\mathrm{NaOH}(1.0 \mathrm{M}, 30 \mathrm{~mL})$ and brine $(20 \mathrm{~mL})$, dried over $\mathrm{Na}_{2} \mathrm{SO}_{4}$ and filtered. After the volatile materials were removed under reduced pressure, the crude residue was purified by column chromatography to give the title compound. Compounds 1f-o were prepared following the General Procedure B.

\section{General procedure $C:^{[3]}$}

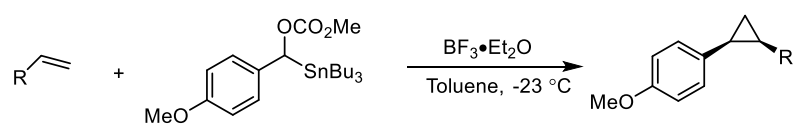

To a $50 \mathrm{~mL}$ oven-dried round-bottom flask equipped with a stir bar was added the solution of (4methoxyphenyl)(tributylstannyl)methyl methyl carbonate $(972 \mathrm{mg}, 2.0 \mathrm{mmol}, 1.0$ equiv) and the corresponding alkene $(2.2 \mathrm{mmol}, 1.1$ equiv) in toluene $(10 \mathrm{~mL})$ under nitrogen atmosphere at room temperature. The reaction vessel was cooled to $-23{ }^{\circ} \mathrm{C} . \mathrm{BF}_{3} \cdot \mathrm{OEt}_{2}$ (156 $\mathrm{mg}, 1.1 \mathrm{mmol}, 1.1$ equiv) was added by syringe and stirred at this temperature for $2 \mathrm{~h}$. After the reaction reached completion according to the TLC analysis, the reaction mixture was quenched with sat. $\mathrm{NaHCO}_{3}(10 \mathrm{~mL})$ and extracted with EtOAc $(30 \mathrm{~mL})$ for 3 times. The combined organic layers were washed with brine $(20 \mathrm{~mL})$, dried over $\mathrm{Na}_{2} \mathrm{SO}_{4}$ and filtered. After the volatile materials were removed under reduced pressure, the crude residue was purified by column chromatography to afford the desired compound. Compounds $\mathbf{1 p - z}$ were prepared following the General Procedure C.

The spectroscopic data of all known cyclopropanes are in accordance with those described in literature. ${ }^{[2]}$

\section{Preparation of hypervalent iodine reagents:}

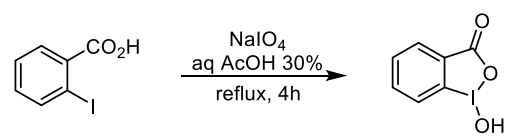




\section{1-Hydroxy-1,2-benziodoxol-3-(1H)-one (BI-OH)}

Following a reported procedure, ${ }^{[4]} \mathrm{NaIO}_{4}(3.62 \mathrm{~g}, 16.9 \mathrm{mmol}, 1.05$ equiv) and 2-iodobenzoic acid (4.00 $\mathrm{g}, 16.1 \mathrm{mmol}, 1.0$ equiv) were suspended in $30 \%$ (v:v) aq. AcOH (30 mL). The mixture was vigorously stirred and refluxed for $4 \mathrm{~h}$. The reaction mixture was then diluted with cold water $(100 \mathrm{~mL})$ and allowed to cool to rt, protecting it from light. After $1 \mathrm{~h}$, the crude product was collected by filtration, washed on the filter with ice water $(3 \times 20 \mathrm{~mL})$ and acetone $(3 \times 20 \mathrm{~mL})$, and air-dried in the dark to give the pure product $\mathrm{BI}-\mathrm{OH}(3.8 \mathrm{~g}, 89 \%)$ as a white solid. The spectroscopic data is in accordance with those described in literature.

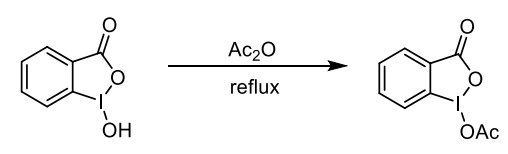

\section{1-Acetoxy-1,2-benziodoxol-3-(1H)-one (BI-OAc)}

Following a reported procedure, ${ }^{[4]} \mathrm{BI}-\mathrm{OH}\left(3.00 \mathrm{~g}, 11.3 \mathrm{mmol}, 1.00\right.$ equiv) was heated in $\mathrm{Ac}_{2} \mathrm{O}(10 \mathrm{~mL})$ to reflux until the solution turned clear (without suspension, ca. $30 \mathrm{~min}$ ). The mixture was then left to cool down and white crystals started to form. The crystallization was continued at $-18{ }^{\circ} \mathrm{C}$. The crystals were then collected and dried overnight under high vacuum to give compound BI-OAc (3.06 g, 10.0 mmol, $86 \%$ yield) as white solid. The spectroscopic data is in accordance with those described in literature.

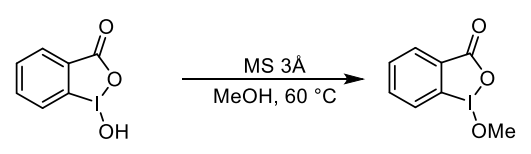

\section{1-Methoxy-benzo[d][1,2]iodaoxol-3(1H)-one (BI-OMe)}

Following a reported procedure ${ }^{[5]}$ to a suspension of $\mathrm{BI}-\mathrm{OH}(264 \mathrm{mg}, 1.0 \mathrm{mmol})$ in an appropriate alcohol $(10 \mathrm{~mL})$ was added MS $3 \AA$ (1 g). After the mixture was stirred under the appropriate conditions, MS $3 \AA$ was filtered using $\mathrm{CH}_{2} \mathrm{Cl}_{2}$, and the solvents were then removed by evaporation. The residue was washed with hexane and filtered to remove the corresponding alcohol completely. The residue was dissolved with $\mathrm{CH}_{2} \mathrm{Cl}_{2}$ and the extract was then filtered through filter paper to remove unreacted substrate. Removal of the solvent by evaporation gave the corresponding benziodoxole alkoxide BI-OMe (212 mg, 75.9\% yield) as a white powder. The spectroscopic data is in accordance with those described in literature.

\section{General procedure D:}




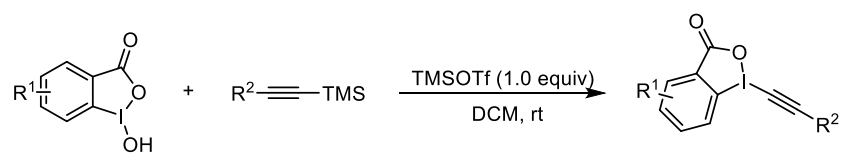

Following a reported procedure ${ }^{[7]}$ trimethylsilyl triflate $(1.0 \mathrm{~mL}, 5.5 \mathrm{mmol}, 1.1$ equiv) was added to a suspension of 2-iodosylbenzoic acid (1.3 g, $5.0 \mathrm{mmol}, 1.0$ equiv) in $\mathrm{CH}_{2} \mathrm{Cl}_{2}(15 \mathrm{~mL})$ at $\mathrm{RT}$. The resulting suspension was stirred for $1 \mathrm{~h}$, followed by the dropwise addition of corresponding alkyne (1.1 equiv), which was dissolved in $\mathrm{CH}_{2} \mathrm{Cl}_{2}(1 \mathrm{~mL})$. The resulting suspension was stirred for $6 \mathrm{~h}$ at room temperature. A saturated solution of $\mathrm{NaHCO}_{3}(20 \mathrm{~mL})$ was then added and the mixture was stirred vigorously for 30 min, the two layers were separated and the organic layer was washed with sat. $\mathrm{NaHCO}_{3}(20 \mathrm{~mL})$, dried over $\mathrm{MgSO}_{4}$, filtered and evaporated under reduced pressure. The resulting solid was boiled in $\mathrm{CH}_{3} \mathrm{CN}$ (20 mL). The mixture was cooled down, filtered and dried under high vacuum to afford EBX 2.

The spectroscopic data of all known EBXs are in accordance with those described in literature. ${ }^{[7]}$

\section{Catalytic results:}

\section{General procedure of catalytic reaction:}

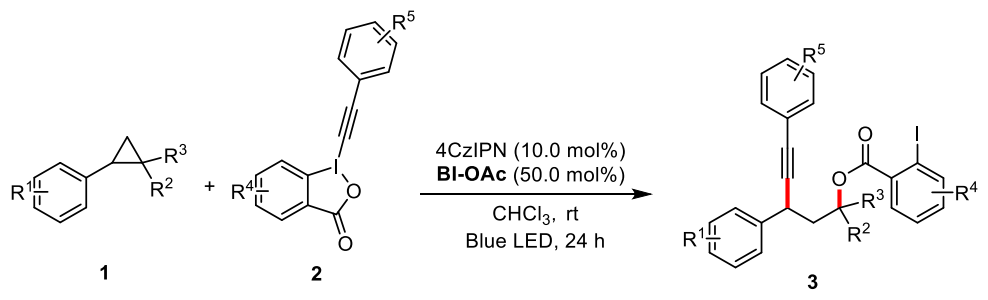

To an oven-dried $10 \mathrm{~mL}$ Schlenk tube equipped with a stir bar, aryl cyclopropanes $2(0.20 \mathrm{mmol}, 1.0$ equiv), EBX 2 (0.30 mmol, 1.5 equiv), photocatalyst 4CzIPN (15.7 mg, $10.0 \mathrm{~mol} \%$ ) and BI-OAc (30.6 $\mathrm{mg}, 50.0 \mathrm{~mol} \%$ ). Then the reaction tube was evacuated and backfilled with argon for two times. Subsequently, anhydrous $\mathrm{CHCl}_{3}(2.0 \mathrm{~mL}, 0.1 \mathrm{M})$ were added. The resulting mixture was irradiated under blue LED at room temperature for $24 \mathrm{~h}$. The residue was purified by silica gel chromatography to afford the desired product 3 .

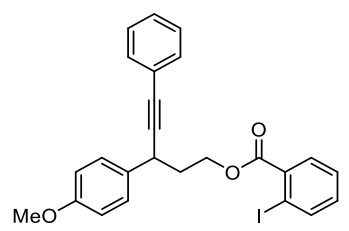

\section{3-(4-Methoxyphenyl)-5-phenylpent-4-yn-1-yl 2-iodobenzoate (3a)}

Purified by flash silica column chromatography (pentane/ethyl acetate $=40: 1$ )

Colorless oil (66.5 mg, $67 \%$ yield) 
${ }^{1} \mathrm{H} \mathrm{NMR}\left(300 \mathrm{MHz}, \mathrm{CDCl}_{3}\right): \delta 7.92(\mathrm{dd}, J=8.0,1.2 \mathrm{~Hz}, 1 \mathrm{H}), 7.69(\mathrm{dd}, J=7.8,1.7 \mathrm{~Hz}, 1 \mathrm{H}), 7.38-7.20$ (m, 8H), $7.07(\mathrm{td}, J=7.6,1.7 \mathrm{~Hz}, 1 \mathrm{H}), 6.86-6.77(\mathrm{~m}, 2 \mathrm{H}), 4.56-4.31(\mathrm{~m}, 2 \mathrm{H}), 4.04(\mathrm{t}, J=7.3 \mathrm{~Hz}, 1 \mathrm{H})$, $3.73(\mathrm{~s}, 3 \mathrm{H}), 2.28-2.09(\mathrm{~m}, 2 \mathrm{H})$

${ }^{13} \mathrm{C}$ NMR $\left(126 \mathrm{MHz}, \mathrm{CDCl}_{3}\right): \delta 166.5,158.7,141.3,135.3,133.1,132.6,131.7,131.1,128.5,128.2$, $127.9,127.8,123.4,114.2,94.0,90.4,83.9,63.7,55.3,37.2,34.5$.

HRMS (ESI) m/z: [M+Na] $]^{+}$Calcd for $\mathrm{C}_{25} \mathrm{H}_{21} \mathrm{INaO}_{3}$ 519.0433; Found: 519.0427.

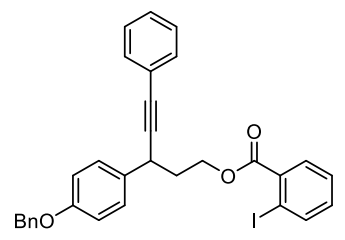

\section{3-(4-(Benzyloxy)phenyl)-5-phenylpent-4-yn-1-yl 2-iodobenzoate (3b)}

Purified by flash silica column chromatography (pentane/ethyl acetate $=40: 1$ )

Colorless oil (81.2 mg, $71 \%$ yield)

${ }^{1} \mathrm{H}$ NMR $\left(300 \mathrm{MHz}, \mathrm{CDCl}_{3}\right): \delta 7.91(\mathrm{dd}, J=8.0,1.2 \mathrm{~Hz}, 1 \mathrm{H}), 7.69(\mathrm{dd}, J=7.8,1.8 \mathrm{~Hz}, 1 \mathrm{H}), 7.38-7.19$ $(\mathrm{m}, 13 \mathrm{H}), 7.09-7.02(\mathrm{~m}, 1 \mathrm{H}), 6.94-6.83(\mathrm{~m}, 2 \mathrm{H}), 4.97(\mathrm{~s}, 2 \mathrm{H}), 4.58-4.32(\mathrm{~m}, 2 \mathrm{H}), 4.03(\mathrm{t}, J=7.4$ $\mathrm{Hz}, 1 \mathrm{H}), 2.29-2.10(\mathrm{~m}, 2 \mathrm{H})$.

${ }^{13} \mathrm{C}$ NMR $\left(76 \mathrm{MHz}, \mathrm{CDCl}_{3}\right) \delta 166.5,157.9,141.3,137.0,135.2,133.3,132.7,131.7,131.1,128.6,128.5$, $128.3,128.0,127.9,127.8,127.5,123.4,115.1,94.1,90.4,83.9,70.1,63.7,37.2,34.6$.

HRMS (ESI) m/z: [M+Na] $]^{+}$Calcd for $\mathrm{C}_{31} \mathrm{H}_{25} \mathrm{INaO}_{3}$ 595.0746; Found: 595.0743.

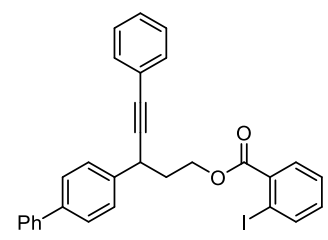

\section{3-([1,1'-Biphenyl]-4-yl)-5-phenylpent-4-yn-1-yl 2-iodobenzoate (3c)}

Purified by flash silica column chromatography (pentane/ethyl acetate $=60: 1$ )

Colorless oil (67.2 mg, $62 \%$ yield)

${ }^{1} \mathrm{H} \mathrm{NMR}\left(300 \mathrm{MHz}, \mathrm{CDCl}_{3}\right): \delta 7.92(\mathrm{dd}, J=8.0,1.2 \mathrm{~Hz}, 1 \mathrm{H}), 7.69(\mathrm{dd}, J=7.8,1.7 \mathrm{~Hz}, 1 \mathrm{H}), 7.50-7.22$ $(\mathrm{m}, 15 \mathrm{H}), 7.07(\mathrm{td}, J=7.7,1.8 \mathrm{~Hz}, 1 \mathrm{H}), 4.60-4.39(\mathrm{~m}, 2 \mathrm{H}), 4.14(\mathrm{t}, J=7.4 \mathrm{~Hz}, 1 \mathrm{H}), 2.29(\mathrm{tq}, J=5.9$, $2.8 \mathrm{~Hz}, 2 \mathrm{H})$.

${ }^{13} \mathrm{C} \mathrm{NMR}\left(151 \mathrm{MHz}, \mathrm{CDCl}_{3}\right): \delta 166.5,141.3,140.8,140.1,140.0,135.2,132.6,131.7,131.0,128.7$, $128.2,128.0,127.9,127.5,127.3,127.1,127.0,123.3,93.9,89.9,84.2,63.6,37.1,35.1$. 
HRMS (ESI) m/z: [M+Na] $]^{+}$Calcd for $\mathrm{C}_{30} \mathrm{H}_{23} \mathrm{INaO}_{2}$ 565.0640; Found: 565.0636.

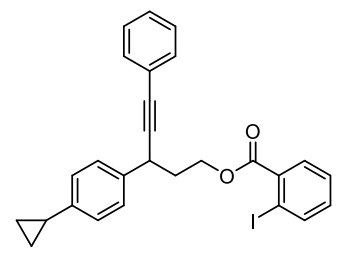

\section{3-(4-Cyclopropylphenyl)-5-phenylpent-4-yn-1-yl 2-iodobenzoate (3d)}

Purified by flash silica column chromatography (pentane/ethyl acetate $=80: 1$ )

Colorless oil (59.8 mg, 59\% yield)

${ }^{1} \mathrm{H}$ NMR (300 MHz, $\left.\mathrm{CDCl}_{3}\right): \delta 7.90(\mathrm{dd}, J=8.0,1.2 \mathrm{~Hz}, 1 \mathrm{H}), 7.67(\mathrm{dd}, J=7.7,1.8 \mathrm{~Hz}, 1 \mathrm{H}), 7.38-7.19$ $(\mathrm{m}, 8 \mathrm{H}), 7.06(\mathrm{td}, J=7.6,1.7 \mathrm{~Hz}, 1 \mathrm{H}), 6.97(\mathrm{~d}, J=8.0 \mathrm{~Hz}, 2 \mathrm{H}), 4.56-4.33(\mathrm{~m}, 2 \mathrm{H}), 4.03(\mathrm{t}, J=7.4 \mathrm{~Hz}$, $1 \mathrm{H}), 2.27-2.12(\mathrm{~m}, 2 \mathrm{H}), 1.80(\mathrm{tt}, J=8.5,5.1 \mathrm{~Hz}, 1 \mathrm{H}), 0.91-0.83(\mathrm{~m}, 2 \mathrm{H}), 0.60(\mathrm{dt}, J=6.8,4.5 \mathrm{~Hz}$, $2 \mathrm{H})$.

${ }^{13} \mathrm{C} \mathrm{NMR}\left(76 \mathrm{MHz}, \mathrm{CDCl}_{3}\right): \delta 166.5,142.9,141.3,137.9,135.2,132.7,131.7,131.1,128.3,128.0,127.9$, $127.4,126.0,123.4,94.1,90.3,83.9,63.7,37.1,34.9,15.1,9.3,9.2$.

HRMS (ESI) m/z: [M+Na] $]^{+}$Calcd for $\mathrm{C}_{27} \mathrm{H}_{23} \mathrm{INaO}_{3}$ 529.0640; Found: 529.0635 .

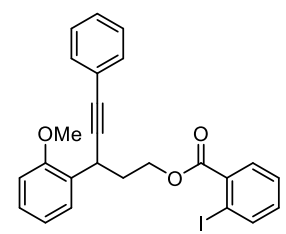

\section{3-(2-Methoxyphenyl)-5-phenylpent-4-yn-1-yl 2-iodobenzoate (3e)}

Purified by flash silica column chromatography (pentane/ethyl acetate $=40: 1$ )

Colorless oil (53.6 mg, 54\% yield)

${ }^{1} \mathrm{H}$ NMR $\left(300 \mathrm{MHz}, \mathrm{CDCl}_{3}\right): \delta 8.02(\mathrm{dd}, J=7.9,1.3 \mathrm{~Hz}, 1 \mathrm{H}), 7.86(\mathrm{dd}, J=7.7,1.7 \mathrm{~Hz}, 1 \mathrm{H}), 7.68(\mathrm{dd}, J$ $=7.5,1.7 \mathrm{~Hz}, 1 \mathrm{H}), 7.50-7.30(\mathrm{~m}, 7 \mathrm{H}), 7.16(\mathrm{td}, J=7.6,1.7 \mathrm{~Hz}, 1 \mathrm{H}), 7.01(\mathrm{td}, J=7.5,1.2 \mathrm{~Hz}, 1 \mathrm{H}), 6.90$ $(\mathrm{dd}, J=8.2,1.2 \mathrm{~Hz}, 1 \mathrm{H}), 4.72-4.46(\mathrm{~m}, 3 \mathrm{H}), 3.84(\mathrm{~s}, 3 \mathrm{H}), 2.45-2.12(\mathrm{~m}, 2 \mathrm{H})$.

${ }^{13} \mathrm{C}$ NMR $\left(126 \mathrm{MHz}, \mathrm{CDCl}_{3}\right): \delta 166.3,156.2,141.4,135.0,132.6,131.7,131.2,130.8,129.3,128.6$, $128.2,128.1,127.8,123.6,120.9,110.5,94.2,90.6,83.3,64.0,55.4,35.4,28.9$.

HRMS (ESI) m/z: [M+Na] ${ }^{+}$Calcd for $\mathrm{C}_{25} \mathrm{H}_{21} \mathrm{INaO}_{3}$ 519.0433; Found: 519.0425 . 


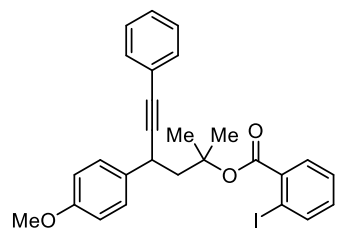

4-(4-Methoxyphenyl)-2-methyl-6-phenylhex-5-yn-2-yl 2-iodobenzoate (3f)

Purified by flash silica column chromatography (pentane/ethyl acetate $=50: 1$ )

Colorless oil (76.4 mg, $73 \%$ yield)

${ }^{1} \mathrm{H}$ NMR $\left(300 \mathrm{MHz}, \mathrm{CDCl}_{3}\right): \delta 7.83(\mathrm{dd}, J=7.9,1.1 \mathrm{~Hz}, 1 \mathrm{H}), 7.59(\mathrm{dd}, J=7.8,1.8 \mathrm{~Hz}, 1 \mathrm{H}), 7.33-7.14$ $(\mathrm{m}, 8 \mathrm{H}), 6.97(\mathrm{td}, J=7.6,1.8 \mathrm{~Hz}, 1 \mathrm{H}), 6.82-6.69(\mathrm{~m}, 2 \mathrm{H}), 3.98(\mathrm{dd}, J=8.7,5.2 \mathrm{~Hz}, 1 \mathrm{H}), 3.68(\mathrm{~s}, 3 \mathrm{H})$, $2.49-2.32(\mathrm{~m}, 2 \mathrm{H}), 1.72(\mathrm{~s}, 3 \mathrm{H}), 1.66(\mathrm{~s}, 3 \mathrm{H})$.

${ }^{13} \mathrm{C} \mathrm{NMR}\left(76 \mathrm{MHz}, \mathrm{CDCl}_{3}\right): \delta 165.9,158.4,141.0,136.6,134.8,132.1,131.5,130.9,128.5,128.2,127.8$, $127.7,123.7,114.1,93.7,92.4,84.1,83.5,55.3,49.1,33.3,27.1,26.6$.

HRMS (ESI) m/z: [M+Na $]^{+}$Calcd for $\mathrm{C}_{27} \mathrm{H}_{25} \mathrm{INaO}_{3}$ 547.0746; Found: 547.0741.

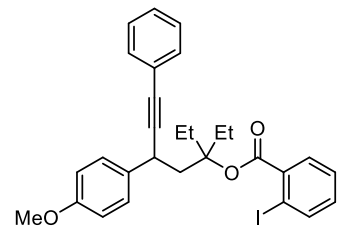

\section{3-Ethyl-5-(4-methoxyphenyl)-7-phenylhept-6-yn-3-yl 2-iodobenzoate (3g)}

Purified by flash silica column chromatography (pentane/ethyl acetate $=40: 1$ )

Colorless oil (67.4 mg, 61\% yield)

${ }^{1} \mathrm{H}$ NMR $\left(300 \mathrm{MHz}, \mathrm{CDCl}_{3}\right): \delta 8.01-7.91(\mathrm{~m}, 1 \mathrm{H}), 7.85-7.69(\mathrm{~m}, 1 \mathrm{H}), 7.48-7.21(\mathrm{~m}, 8 \mathrm{H}), 7.09(\mathrm{td}$, $J=7.6,1.7 \mathrm{~Hz}, 1 \mathrm{H}), 6.88(\mathrm{dd}, J=7.6,5.4 \mathrm{~Hz}, 2 \mathrm{H}), 4.03-3.83(\mathrm{~m}, 1 \mathrm{H}), 3.81(\mathrm{~s}, 3 \mathrm{H}), 2.75-2.33(\mathrm{~m}$, 2H), $2.36-1.79(\mathrm{~m}, 4 \mathrm{H}), 1.01(\mathrm{dt}, J=12.2,7.5 \mathrm{~Hz}, 6 \mathrm{H})$.

${ }^{13} \mathrm{C}$ NMR $\left(76 \mathrm{MHz}, \mathrm{CDCl}_{3}\right) \delta 165.4,158.4,141.3,136.1,134.9,132.2,131.5,131.1,128.5,128.1,127.7$, $127.6,123.7,114.0,94.0,92.1,89.8,83.2,55.3,42.7,32.8,27.6,27.3,8.2,7.9$.

HRMS (ESI) m/z: [M+Na] ${ }^{+}$Calcd for $\mathrm{C}_{29} \mathrm{H}_{29} \mathrm{INaO}_{3}$ 575.1059; Found: 575.1055 .

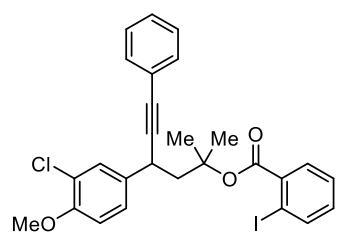

4-(3-Chloro-4-methoxyphenyl)-2-methyl-6-phenylhex-5-yn-2-yl 2-iodobenzoate (3h)

Purified by flash silica column chromatography (pentane/ethyl acetate $=40: 1$ ) 
Colorless oil (59.2 mg, 53\% yield)

${ }^{1} \mathrm{H}$ NMR $\left(300 \mathrm{MHz}, \mathrm{CDCl}_{3}\right): \delta 7.87-7.79(\mathrm{~m}, 1 \mathrm{H}), 7.59(\mathrm{dd}, J=7.8,1.8 \mathrm{~Hz}, 1 \mathrm{H}), 7.38(\mathrm{~d}, J=2.3 \mathrm{~Hz}$, 1H), $7.26-7.16(\mathrm{~m}, 7 \mathrm{H}), 7.00(\mathrm{td}, J=7.7,1.8 \mathrm{~Hz}, 1 \mathrm{H}), 6.78(\mathrm{dd}, J=8.4,6.7 \mathrm{~Hz}, 1 \mathrm{H}), 3.96(\mathrm{t}, J=6.9$ $\mathrm{Hz}, 1 \mathrm{H}), 3.77$ (s, 3H), $2.41(\mathrm{~d}, J=7.0 \mathrm{~Hz}, 2 \mathrm{H}), 1.73(\mathrm{~s}, 3 \mathrm{H}), 1.66(\mathrm{~s}, 3 \mathrm{H})$. ${ }^{13} \mathrm{C} \mathrm{NMR}\left(76 \mathrm{MHz}, \mathrm{CDCl}_{3}\right): \delta 165.9,153.8,141.1,136.4,135.8,132.2,131.5,130.9,129.3,128.2,127.9$, $127.8,126.7,123.4,122.4,112.1,93.7,91.5,83.9,56.2,48.7,33.1,27.2,26.5$.

HRMS (ESI) m/z: [M+Na] $]^{+}$Calcd for $\mathrm{C}_{27} \mathrm{H}_{24} \mathrm{ClINaO}_{3}$ 581.0356; Found: 581.0355 .

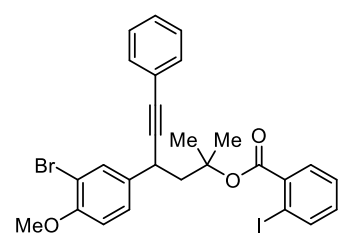

\section{4-(3-Bromo-4-methoxyphenyl)-2-methyl-6-phenylhex-5-yn-2-yl 2-iodobenzoate (3i)}

Purified by flash silica column chromatography (pentane/ethyl acetate $=40: 1$ )

Colorless oil (67.6 mg, 56\% yield)

${ }^{1} \mathrm{H}$ NMR $\left(300 \mathrm{MHz}, \mathrm{CDCl}_{3}\right): \delta 7.94(\mathrm{~d}, J=7.9 \mathrm{~Hz}, 1 \mathrm{H}), 7.72-7.63(\mathrm{~m}, 2 \mathrm{H}), 7.38-7.28(\mathrm{~m}, 6 \mathrm{H}), 7.19$ $-7.03(\mathrm{~m}, 2 \mathrm{H}), 6.84(\mathrm{~d}, J=8.4 \mathrm{~Hz}, 1 \mathrm{H}), 4.06(\mathrm{t}, J=6.9 \mathrm{~Hz}, 1 \mathrm{H}), 3.91-3.83(\mathrm{~m}, J=12.7 \mathrm{~Hz}, 4 \mathrm{H}), 2.50$ $(\mathrm{d}, J=6.9 \mathrm{~Hz}, 2 \mathrm{H}), 1.82(\mathrm{~s}, 3 \mathrm{H}), 1.76(\mathrm{~s}, 3 \mathrm{H})$.

${ }^{13} \mathrm{C} \mathrm{NMR}\left(76 \mathrm{MHz}, \mathrm{CDCl}_{3}\right): \delta 154.7,146.9,141.1,136.4,136.2,132.3,132.2,131.5,130.9,128.6,128.2$, $127.9,127.8,127.5,123.4,111.9,111.6,93.7,91.5,83.9,56.3,48.8,33.0,27.2,26.5$.

HRMS (ESI) m/z: [M+Na $]^{+}$Calcd for $\mathrm{C}_{27} \mathrm{H}_{24} \mathrm{BrINaO}_{3}$ 624.9851; Found: 624.9850 .

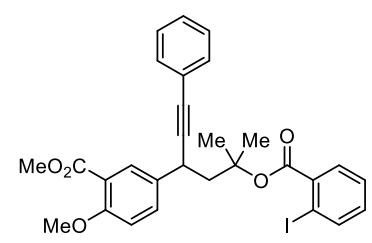

\section{Methyl 5-(5-((2-iodobenzoyl)oxy)-5-methyl-1-phenylhex-1-yn-3-yl)-2-methoxybenzoate (3j)}

Purified by flash silica column chromatography (pentane/ethyl acetate $=20: 1$ )

Colorless oil (68.7 mg, $59 \%$ yield)

${ }^{1} \mathrm{H}$ NMR $\left(300 \mathrm{MHz}, \mathrm{CDCl}_{3}\right): \delta 7.96-7.86(\mathrm{~m}, 2 \mathrm{H}), 7.64(\mathrm{ddd}, J=30.7,8.2,2.1 \mathrm{~Hz}, 2 \mathrm{H}), 7.36-7.23$ (m, 6H), $7.09(\mathrm{td}, J=7.6,1.8 \mathrm{~Hz}, 1 \mathrm{H}), 6.94(\mathrm{~d}, J=8.6 \mathrm{~Hz}, 1 \mathrm{H}), 4.12(\mathrm{~d}, J=5.1 \mathrm{~Hz}, 1 \mathrm{H}), 3.91(\mathrm{~s}, 3 \mathrm{H})$, $3.89(\mathrm{~s}, 3 \mathrm{H}), 2.59-2.46(\mathrm{~m}, 2 \mathrm{H}), 1.83(\mathrm{~s}, 3 \mathrm{H}), 1.76(\mathrm{~s}, 3 \mathrm{H})$. 
${ }^{13} \mathrm{C} \mathrm{NMR}\left(76 \mathrm{MHz}, \mathrm{CDCl}_{3}\right): \delta 166.5,165.9,158.0,141.1,136.4,134.4,132.6,132.2,131.6,131.5,130.9$, $130.7,128.2,127.9,127.8,123.4,119.9,112.4,93.7,91.6,83.9,56.1,52.1,48.7,33.1,27.1,26.5$. HRMS (ESI) m/z: [M+Na] $]^{+}$Calcd for $\mathrm{C}_{29} \mathrm{H}_{27} \mathrm{INaO}_{5}$ 605.0801; Found: 605.0778 .

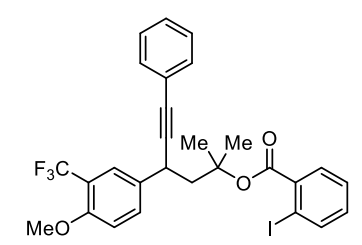

4-(4-Methoxy-3-(trifluoromethyl)phenyl)-2-methyl-6-phenylhex-5-yn-2-yl 2-iodobenzoate (3k)

Purified by flash silica column chromatography (pentane/ethyl acetate $=40: 1$ )

Colorless oil (64.0 mg, 54\% yield)

${ }^{1} \mathrm{H}$ NMR $\left(300 \mathrm{MHz}, \mathrm{CDCl}_{3}\right): \delta 7.98-7.92(\mathrm{~m}, 1 \mathrm{H}), 7.70-7.64(\mathrm{~m}, 2 \mathrm{H}), 7.62-7.58(\mathrm{~m}, 1 \mathrm{H}), 7.37-$ $7.33(\mathrm{~m}, 2 \mathrm{H}), 7.30-7.26(\mathrm{~m}, 4 \mathrm{H}), 7.14-7.03(\mathrm{~m}, 1 \mathrm{H}), 6.96(\mathrm{~d}, J=8.6 \mathrm{~Hz}, 1 \mathrm{H}), 4.13(\mathrm{t}, J=6.9 \mathrm{~Hz}, 1 \mathrm{H})$, $3.88(\mathrm{~s}, 3 \mathrm{H}), 2.52(\mathrm{~d}, J=7.2 \mathrm{~Hz}, 2 \mathrm{H}), 1.85(\mathrm{~s}, 3 \mathrm{H}), 1.77(\mathrm{~s}, 3 \mathrm{H})$.

${ }^{13} \mathrm{C}$ NMR $\left(126 \mathrm{MHz}, \mathrm{CDCl}_{3}\right)\left\{{ }^{19} \mathrm{~F}\right\}: \delta 165.9,156.3,141.1,136.4,134.4,132.2,132.2,131.4,130.8$, $128.2,127.9,127.8,126.2,123.6,123.3,118.7,112.3,93.6,91.4,84.1,83.9,56.0,48.8,33.2,27.2,26.5$. ${ }^{19} \mathrm{~F}$ NMR $\left(282 \mathrm{MHz}, \mathrm{CDCl}_{3}\right): \delta-62.28$.

HRMS (ESI) m/z: [M+Na $]^{+}$Calcd for $\mathrm{C}_{28} \mathrm{H}_{24} \mathrm{~F}_{3} \mathrm{INaO}_{3}$ 615.0620; Found: 615.0613.

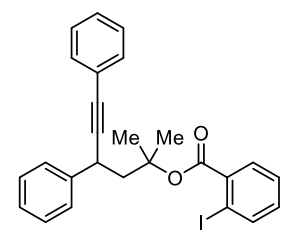

\section{2-Methyl-4,6-diphenylhex-5-yn-2-yl 2-iodobenzoate (3I)}

Purified by flash silica column chromatography (pentane/ethyl acetate $=80: 1$ )

Colorless oil (44.5 mg, $45 \%$ yield)

${ }^{1} \mathrm{H}$ NMR $\left(300 \mathrm{MHz}, \mathrm{CDCl}_{3}\right): \delta 7.89-7.81(\mathrm{~m}, 1 \mathrm{H}), 7.65-7.57(\mathrm{~m}, 1 \mathrm{H}), 7.40-7.16(\mathrm{~m}, 11 \mathrm{H}), 6.99(\mathrm{td}$, $J=7.7,1.8 \mathrm{~Hz}, 1 \mathrm{H}), 4.04(\mathrm{dd}, J=9.4,4.4 \mathrm{~Hz}, 1 \mathrm{H}), 2.43(\mathrm{qd}, J=14.2,6.9 \mathrm{~Hz}, 2 \mathrm{H}), 1.75(\mathrm{~s}, 3 \mathrm{H}), 1.68(\mathrm{~s}$, $3 \mathrm{H})$.

${ }^{13} \mathrm{C}$ NMR $\left(151 \mathrm{MHz}, \mathrm{CDCl}_{3}\right): \delta 165.9,142.7,141.0,136.7,133.1,132.1,131.4,130.8,128.7,128.1$, $127.8,127.7,127.5,126.8,123.6,93.6,91.9,84.1,83.7,49.1,34.1,27.1,26.5$.

HRMS (ESI) m/z: [M+Na] $]^{+}$Calcd for $\mathrm{C}_{26} \mathrm{H}_{23} \mathrm{INaO}_{2}$ 517.0640; Found: 517.0632 . 


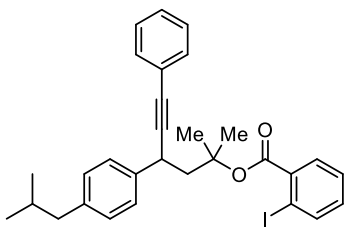

4-(4-Isobutylphenyl)-2-methyl-6-phenylhex-5-yn-2-yl 2-iodobenzoate (3m)

Purified by flash silica column chromatography (pentane/ethyl acetate $=80: 1$ )

Colorless oil (52.9 mg, $48 \%$ yield)

${ }^{1} \mathrm{H}$ NMR $\left(300 \mathrm{MHz}, \mathrm{CDCl}_{3}\right): \delta 7.96(\mathrm{td}, J=8.8,7.9,2.5 \mathrm{~Hz}, 1 \mathrm{H}), 7.73(\mathrm{dd}, J=7.8,1.7 \mathrm{~Hz}, 1 \mathrm{H}), 7.53-$ $7.26(\mathrm{~m}, 8 \mathrm{H}), 7.21-7.00(\mathrm{~m}, 3 \mathrm{H}), 4.10(\mathrm{dd}, J=9.5,4.3 \mathrm{~Hz}, 1 \mathrm{H}), 2.66-2.37(\mathrm{~m}, 4 \mathrm{H}), 1.84(\mathrm{~s}, 3 \mathrm{H}), 1.77$ (s, 3H), $1.52-1.25(\mathrm{~m}, 1 \mathrm{H}), 0.92(\mathrm{~s}, 3 \mathrm{H}), 0.90(\mathrm{~s}, 3 \mathrm{H})$.

${ }^{13} \mathrm{C}$ NMR $\left(126 \mathrm{MHz}, \mathrm{CDCl}_{3}\right): \delta 165.9,141.0,140.2,139.9,136.7,132.0,131.4,130.8,129.4,128.1$, $127.8,127.7,127.2,123.7,93.7,92.3,84.2,83.6,49.1,45.0,33.7,30.2,27.0,26.5,22.4,22.3$.

HRMS (ESI) m/z: [M+Na] ${ }^{+}$Calcd for $\mathrm{C}_{30} \mathrm{H}_{31} \mathrm{INaO}_{2}$ 573.1266; Found: 573.1260.

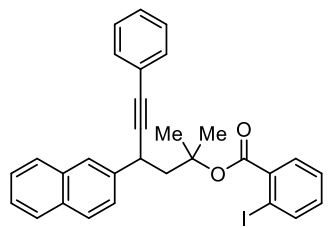

\section{2-Methyl-4-(naphthalen-2-yl)-6-phenylhex-5-yn-2-yl 2-iodobenzoate (3n)}

Purified by flash silica column chromatography (pentane/ethyl acetate $=80: 1$ )

Colorless oil (59.9 mg, 55\% yield)

${ }^{1} \mathrm{H}$ NMR $\left(300 \mathrm{MHz}, \mathrm{CDCl}_{3}\right): \delta 7.84-7.80(\mathrm{~m}, 2 \mathrm{H}), 7.76-7.70(\mathrm{~m}, 3 \mathrm{H}), 7.54-7.49(\mathrm{~m}, 3 \mathrm{H}), 7.41-$ $7.27(\mathrm{~m}, 6 \mathrm{H}), 7.12-7.06(\mathrm{~m}, 1 \mathrm{H}), 6.96(\mathrm{td}, J=7.7,1.8 \mathrm{~Hz}, 1 \mathrm{H}), 4.21(\mathrm{t}, J=6.9 \mathrm{~Hz}, 1 \mathrm{H}), 2.53(\mathrm{~d}, J=$ $6.9 \mathrm{~Hz}, 2 \mathrm{H}), 1.78(\mathrm{~s}, 3 \mathrm{H}), 1.70(\mathrm{~s}, 3 \mathrm{H})$.

${ }^{13} \mathrm{C}$ NMR $\left(151 \mathrm{MHz}, \mathrm{CDCl}_{3}\right): \delta 165.9,140.9,139.9,136.5,133.5,132.5,132.0,131.5,130.8,128.5$, $128.2,127.8,127.7,127.6,127.5,126.1,125.9,125.9,125.6,123.6,93.6,91.9,84.1,83.9,48.7,34.3$, 27.1, 26.5.

HRMS (ESI) m/z: [M+Na] Calcd for $\mathrm{C}_{30} \mathrm{H}_{25} \mathrm{INaO}_{2}$ 567.0797; Found: 567.0790.

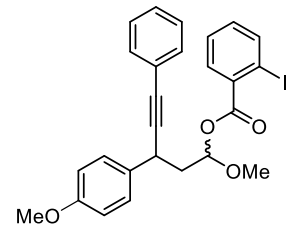

1-Methoxy-3-(4-methoxyphenyl)-5-phenylpent-4-yn-1-yl 2-iodobenzoate (3o) 
Purified by flash silica column chromatography (pentane/ethyl acetate $=20: 1$ ) as inseparable mixture of diastereoisomers $(\mathrm{dr}=1.3: 1)$

Colorless oil (52.6 mg, 50\% yield)

${ }^{1} \mathrm{H}$ NMR (300 MHz, $\left.\mathrm{CDCl}_{3}\right)$ : (mixture of diastereoisomers) $\delta 7.95-7.85(\mathrm{~m}, 1 \mathrm{H}), 7.80-7.69(\mathrm{~m}, 1 \mathrm{H})$, $7.41-7.18(\mathrm{~m}, 8 \mathrm{H}), 7.06(\mathrm{td}, J=7.5,1.8 \mathrm{~Hz}, 1 \mathrm{H}), 6.87-6.76(\mathrm{~m}, 2 \mathrm{H}),[6.26-6.23(\mathrm{~m}$, minor diastereoisomer $)+5.98(\mathrm{t}, J=5.7 \mathrm{~Hz}$, major diastereoisomer $), 1 \mathrm{H}], 4.11-3.92(\mathrm{~m}, 1 \mathrm{H}), 3.72(\mathrm{~d}, J=4.2$ $\mathrm{Hz}, 3 \mathrm{H}),[3.55(\mathrm{~s})+3.46(\mathrm{~s}), 3 \mathrm{H}], 2.41-2.15(\mathrm{~m}, 2 \mathrm{H})$.

${ }^{13} \mathrm{C}$ NMR (76 MHz, $\mathrm{CDCl}_{3}$ ): (mixture of diastereoisomers) $\delta 166.0,165.9,158.7,158.6,141.5,141.4$, $134.6,134.5,133.1,132.9,132.8,132.7,131.8,131.7,131.2,131.1,128.8,128.6,128.5,128.3,128.2$, 128.0, 127.9, 127.8, 127.7, 123.5, 123.4, 114.2, 114.1, 99.9, 99.3, 94.4, 94.3, 90.7, 89.9, 83.9, 83.4, 57.9, $57.3,55.3,42.9,42.4,33.4,33.2$.

HRMS (ESI) m/z: [M+Na] $]^{+}$Calcd for $\mathrm{C}_{26} \mathrm{H}_{23} \mathrm{INaO}_{4}$ 549.0539; Found: 549.0534.

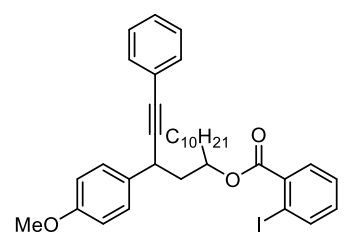

\section{3-(4-Methoxyphenyl)-1-phenylpentadec-1-yn-5-yl 2-iodobenzoate (3p)}

Purified by flash silica column chromatography (pentane/ethyl acetate $=40: 1$ ) as inseparable mixture of diastereoisomers $(\mathrm{dr}=1.2: 1)$

Colorless oil ( $80.2 \mathrm{mg}, 63 \%$ yield)

${ }^{1} \mathrm{H} \mathrm{NMR}\left(300 \mathrm{MHz}, \mathrm{CDCl}_{3}\right)$ : (mixture of diastereoisomers) $\delta 7.91$ (dd, $\left.J=8.0,1.7 \mathrm{~Hz}, 1 \mathrm{H}\right), 7.66$ (ddd, $J=10.0,7.7,1.7 \mathrm{~Hz}, 1 \mathrm{H}), 7.40-7.18(\mathrm{~m}, 8 \mathrm{H}), 7.11-7.01(\mathrm{~m}, 1 \mathrm{H}), 6.89-6.69(\mathrm{~m}, 2 \mathrm{H}),[5.58-5.36$ $(\mathrm{m}$, major diastereoisomer $)+5.18-5.13(\mathrm{~m}$, minor diastereoisomer $), 1 \mathrm{H}] .4 .09-3.80(\mathrm{~m}, 1 \mathrm{H}), 3.71(\mathrm{~d}$, $J=2.7 \mathrm{~Hz}, 3 \mathrm{H}), 2.37-1.92(\mathrm{~m}, 2 \mathrm{H}), 1.69(\mathrm{dq}, J=15.5,8.1 \mathrm{~Hz}, 2 \mathrm{H}), 1.18(\mathrm{~d}, J=13.2 \mathrm{~Hz}, 16 \mathrm{H}), 0.80(\mathrm{t}$, $J=6.5 \mathrm{~Hz}, 3 \mathrm{H})$

${ }^{13} \mathrm{C}$ NMR (151 MHz, $\mathrm{CDCl}_{3}$ ): (mixture of diastereoisomers) $\delta 166.2,165.9,158.6,158.5,141.3,141.1$, 136.2, 135.5, 133.7, 133.2, 132.4, 132.3, 131.7, 131.6, 131.6, 130.9, 130.6, 128.6, 128.3, 128.2, 128.1, $128.0,127.9,127.8,127.7,127.6,123.5,114.1,114.0,94.0,93.7,91.4,90.4,84.1,83.1,74.7,74.0,55.3$, $55.2,43.0,42.5,34.6,34.6,34.3,34.2,31.9,29.6,29.5,29.5,29.4,29.4,29.4,29.3,29.2,25.2,25.1$, 22.7, 14.1 . 
HRMS (ESI) m/z: [M+Na] $]^{+}$Calcd for $\mathrm{C}_{35} \mathrm{H}_{41} \mathrm{INaO}_{3}$ 659.1998; Found: 659.1999.

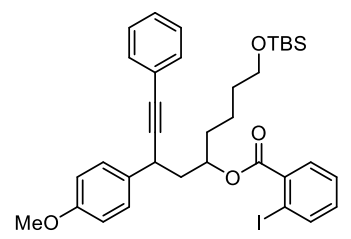

\section{9-((tert-Butyldimethylsilyl)oxy)-3-(4-methoxyphenyl)-1-phenylnon-1-yn-5-yl 2-iodobenzoate (3q)}

Purified by flash silica column chromatography (pentane/ethyl acetate $=40: 1$ ) as inseparable mixture of diastereoisomers $(\mathrm{dr}=1.8: 1)$

Colorless oil (91.4 mg, 67\% yield)

${ }^{1} \mathrm{H}$ NMR (300 MHz, $\mathrm{CDCl}_{3}$ ): (mixture of diastereoisomers) $\delta 7.95$ (dt, $\left.J=7.9,1.4 \mathrm{~Hz}, 1 \mathrm{H}\right), 7.72$ (ddd, $J=10.6,7.8,1.7 \mathrm{~Hz}, 1 \mathrm{H}), 7.47-7.23(\mathrm{~m}, 8 \mathrm{H}), 7.10(\mathrm{tdd}, J=7.7,3.1,1.8 \mathrm{~Hz}, 1 \mathrm{H}), 6.85(\mathrm{dt}, J=8.8,2.8$ $\mathrm{Hz}, 2 \mathrm{H}),[5.58-5.43(\mathrm{~m}$, major diastereoisomer $)+5.25-5.14(\mathrm{~m}$, minor diastereoisomer) $1 \mathrm{H}], 4.09-$ $3.87(\mathrm{~m}, 1 \mathrm{H}), 3.76(\mathrm{~s}, 3 \mathrm{H}), 3.57(\mathrm{q}, J=5.8 \mathrm{~Hz}, 2 \mathrm{H}), 2.44-2.01(\mathrm{~m}, 2 \mathrm{H}), 1.79-1.41(\mathrm{~m}, 6 \mathrm{H}), 0.84(\mathrm{~s}$, 9H) $-0.10(\mathrm{~s}, 6 \mathrm{H})$.

${ }^{13} \mathrm{C} \mathrm{NMR}\left(76 \mathrm{MHz}, \mathrm{CDCl}_{3}\right)$ : (mixture of diastereoisomers) $\delta 166.2,166.0,158.6,158.5,141.3,141.2$, 136.0, 133.7, 132.5, 132.4, 131.8, 131.7, 130.9, 130.7, 128.7, 128.4, 128.2, 127.9, 127.8, 123.5, 114.1, 114.0, 94.1, 93.9, 91.3, 90.4, 85.1, 84.1, 74.6, 73.9, 63.0, 62.9, 55.3, 55.2, 42.9, 42.5, 34.6, 34.4, 34.3, $33.9,32.7,32.6,26.0,21.6,21.5,18.4,-5.2$

HRMS (ESI) m/z: [M+Na] $]^{+}$Calcd for $\mathrm{C}_{35} \mathrm{H}_{43} \mathrm{INaO}_{4} \mathrm{Si}$ 705.1873; Found: 705.1861.

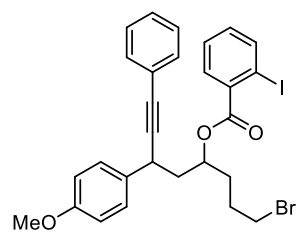

\section{1-Bromo-6-(4-methoxyphenyl)-8-phenyloct-7-yn-4-yl 2-iodobenzoate (3r)}

Purified by flash silica column chromatography (pentane/ethyl acetate $=30: 1$ ) as inseparable mixture of diastereoisomers $(\mathrm{dr}=1.7: 1)$

Colorless oil (72.8 mg, 59\% yield)

${ }^{1} \mathrm{H}$ NMR (300 MHz, $\mathrm{CDCl}_{3}$ ): (mixture of diastereoisomers) $\delta 7.90(\mathrm{dt}, J=7.9,1.6 \mathrm{~Hz}, 1 \mathrm{H}), 7.73-7.56$ (m, 1H), $7.37-7.17(\mathrm{~m}, 8 \mathrm{H}), 7.05$ (tdd, $J=7.6,3.8,1.7 \mathrm{~Hz}, 1 \mathrm{H}), 6.79(\mathrm{dd}, J=8.8,2.8 \mathrm{~Hz}, 2 \mathrm{H}),[5.53-$ $5.45(\mathrm{~m}$, major diastereoisomer $)+5.25-5.10(\mathrm{~m}$, minor diastereoisomer $), 1 \mathrm{H}], 4.02-3.82(\mathrm{~m}, 1 \mathrm{H})$, $3.70(\mathrm{~s}, 3 \mathrm{H}), 3.44-3.19(\mathrm{~m}, 2 \mathrm{H}), 2.39-1.80(\mathrm{~m}, 6 \mathrm{H})$ 
${ }^{13} \mathrm{C}$ NMR (76 MHz, $\mathrm{CDCl}_{3}$ ): (mixture of diastereoisomers) $\delta 166.2,165.9,158.7,158.6,141.4,141.3$, $135.7,133.4,132.9,132.7,132.6,131.8,131.7,130.9,130.8,128.6,128.4,128.2,128.0,127.9,123.4$, 114.2, 114.1, 94.2, 93.9, 91.0, 90.2, 84.3, 83.4, 73.5, 72.9, 55.4, 55.3, 43.1, 42.6, 34.6, 34.3, 33.4, 33.3, $32.8,28.5,28.4$

HRMS (ESI) m/z: [M+Na] Calcd for $\mathrm{C}_{28} \mathrm{H}_{26} \mathrm{BrINaO}_{3}$ 639.0008; Found: 639.0007.

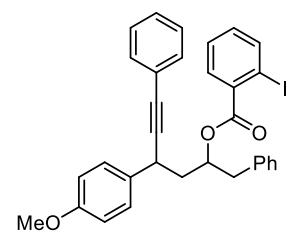

4-(4-Methoxyphenyl)-1,6-diphenylhex-5-yn-2-yl 2-iodobenzoate (3s)

Purified by flash silica column chromatography (pentane/ethyl acetate $=40: 1$ ) as inseparable mixture of diastereoisomers $(\mathrm{dr}=1.5: 1)$

Colorless oil (57.5 mg, $49 \%$ yield)

${ }^{1} \mathrm{H} \mathrm{NMR}\left(300 \mathrm{MHz}, \mathrm{CDCl}_{3}\right)$ : (mixture of diastereoisomers) $\delta 8.02-7.97(\mathrm{~m}, 1 \mathrm{H}), 7.75-7.27(\mathrm{~m}, 14 \mathrm{H})$, $7.19-7.12(\mathrm{~m}, 1 \mathrm{H}), 6.88(\mathrm{dt}, J=8.7,3.1 \mathrm{~Hz}, 2 \mathrm{H}),[5.90-5.66(\mathrm{~m}$, major diastereoisomer $)+5.51-5.39$ (m, minor diastereoisomer), $1 \mathrm{H}], 4.14-3.89(\mathrm{~m}, 1 \mathrm{H}), 3.80(\mathrm{~s}, 3 \mathrm{H}), 3.28-2.99(\mathrm{~m}, 2 \mathrm{H}), 2.48-2.02(\mathrm{~m}$, $2 \mathrm{H})$.

${ }^{13} \mathrm{C}$ NMR (76 MHz, $\mathrm{CDCl}_{3}$ ): (mixture of diastereoisomers) $\delta 166.1,165.8,158.6,158.5,141.3,141.2$, $141.1,136.8,135.8,135.1,133.5,132.9,132.5,132.4,131.8,131.7,131.6,130.9,130.7,129.8,129.7$, $129.6,129.5,129.2,128.6,128.5,128.5,128.4,128.3,128.2,127.9,127.8,127.7,127.6,126.7,123.5$, 114.1, 114.1, 114.0, 113.9, 94.2, 93.9, 91.2, 90.2, 84.2, 83.2, 75.0, 74.2, 55.3, 55.2, 42.2, 41.6, 40.8, 40.4, $34.6,34.3$.

HRMS (ESI) m/z: [M+Na] $]^{+}$Calcd for $\mathrm{C}_{32} \mathrm{H}_{27} \mathrm{INaO}_{3}$ 609.0903; Found: 609.0894.

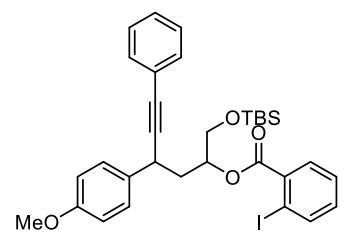

1-((tert-Butyldimethylsilyl)oxy)-4-(4-methoxyphenyl)-6-phenylhex-5-yn-2-yl 2-iodobenzoate (3t)

Purified by flash silica column chromatography (pentane/ethyl acetate $=40: 1$ ) as inseparable mixture of diastereoisomers $(\mathrm{dr}=1.3: 1)$

Colorless oil ( $84.5 \mathrm{mg}, 66 \%$ yield) 
${ }^{1} \mathrm{H}$ NMR $\left(300 \mathrm{MHz}, \mathrm{CDCl}_{3}\right)$ : (mixture of diastereoisomers) $\delta 8.07-7.86(\mathrm{~m}, 1 \mathrm{H}), 7.81-7.64(\mathrm{~m}, 1 \mathrm{H})$, $7.45-7.23(\mathrm{~m}, 8 \mathrm{H}), 7.13-7.06(\mathrm{~m}, 1 \mathrm{H}), 6.94-6.74(\mathrm{~m}, 2 \mathrm{H}),[5.52-5.45(\mathrm{~m}$, minor diastereoisomer $)$ $+5.38-5.16(\mathrm{~m}$, major diastereoisomer $), 1 \mathrm{H}], 4.55-3.81(\mathrm{~m}, 3 \mathrm{H}), 3.77-3.71(\mathrm{~m}, 3 \mathrm{H}), 2.50-2.01(\mathrm{~m}$, $2 \mathrm{H}), 0.88-0.74(\mathrm{~m}, 9 \mathrm{H}),-0.06(\mathrm{~d}, J=2.3 \mathrm{~Hz}, 6 \mathrm{H})$.

${ }^{13} \mathrm{C}$ NMR (76 MHz, $\mathrm{CDCl}_{3}$ ): (mixture of diastereoisomers) $\delta 166.0,165.8,158.6,158.6,141.4,141.3$, $135.6,135.1,133.7,133.3,132.6,132.5,131.8,131.7,131.6,131.5,131.1,131.0,130.9,130.8,130.5$, 129.2, 128.6, 128.6, 128.4, 128.3, 128.2, 127.9, 127.8, 127.7, 123.5, 114.1, 114.1, 113.9, 113.8, 94.2, $94.0,91.2,90.5,84.1,83.3,74.7,74.2,64.2,63.7,55.3,39.6,39.2,34.4,34.2,26.0,25.9,25.8,18.3,-$ $5.3,-5.2$

HRMS (ESI) m/z: [M+Na] $]^{+}$Calcd for $\mathrm{C}_{32} \mathrm{H}_{37} \mathrm{INaO}_{4} \mathrm{Si}$ 663.1403; Found: 663.1397.

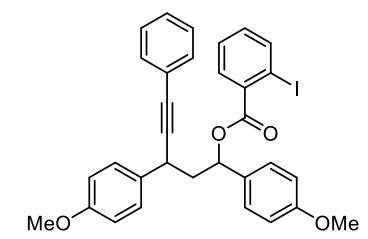

\section{1,3-Bis(4-methoxyphenyl)-5-phenylpent-4-yn-1-yl 2-iodobenzoate (3u)}

Purified by flash silica column chromatography (pentane/ethyl acetate $=20: 1$ ) as inseparable mixture of diastereoisomers $(\mathrm{dr}=1.4: 1)$

Colorless oil (80.7 mg, 67\% yield)

${ }^{1} \mathrm{H}$ NMR (300 MHz, $\left.\mathrm{CDCl}_{3}\right)$ : (mixture of diastereoisomers) $\delta 7.94-7.60(\mathrm{~m}, 2 \mathrm{H}), 7.41-7.12(\mathrm{~m}, 10 \mathrm{H})$, $7.00-6.72(\mathrm{~m}, 5 \mathrm{H}),[6.22-6.10(\mathrm{~m}$, major diastereoisomer $)+6.06-5.95(\mathrm{~m}$, minor diastereoisomer $)$, $1 \mathrm{H}], 3.98(\mathrm{dd}, J=9.7,5.8 \mathrm{~Hz}, 1 \mathrm{H}), 3.74-3.56(\mathrm{~m}, 6 \mathrm{H}), 2.71-2.42(\mathrm{~m}, 1 \mathrm{H}), 2.31-2.12(\mathrm{~m}, 1 \mathrm{H})$.

${ }^{13} \mathrm{C}$ NMR (76 MHz, $\mathrm{CDCl}_{3}$ ): (mixture of diastereoisomers) $\delta 165.6,165.4,159.7,159.6,158.7,141.4$, $141.3,135.5,135.1,133.3,133.1,132.7,132.6,132.0,131.8,131.7,131.5,131.2,131.1,128.6,128.5$, $128.4,128.3,128.2,127.9,123.5,114.2,114.1,114.0,94.2,94.0,90.7,90.6,84.1,83.9,75.9,75.8,55.4$, $44.9,44.6,34.7,34.4$

HRMS (ESI) m/z: [M+Na] $]^{+}$Calcd for $\mathrm{C}_{32} \mathrm{H}_{27} \mathrm{INaO}_{4}$ 625.0852; Found: 625.0849 .

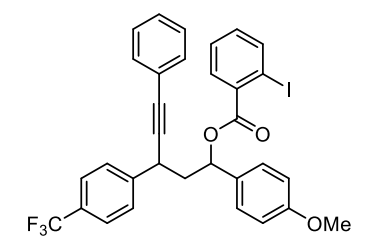

1-(4-Methoxyphenyl)-5-phenyl-3-(4-(trifluoromethyl)phenyl)pent-4-yn-1-yl 2-iodobenzoate (3v) 
Purified by flash silica column chromatography (pentane/ethyl acetate $=30: 1$ ) as inseparable mixture of diastereoisomers $(\mathrm{dr}=2: 1)$ and regioisomeric $(10: 1 \mathrm{rr})$

Colorless oil (79.4 mg, $62 \%$ yield)

${ }^{1} \mathrm{H}$ NMR (300 MHz, $\left.\mathrm{CDCl}_{3}\right)$ : (mixture of diastereoisomers and regioisomer) $\delta 7.94-7.86(\mathrm{~m}, 1 \mathrm{H})$, $7.71-7.60(\mathrm{~m}, 1 \mathrm{H}), 7.50-7.22(\mathrm{~m}, 12 \mathrm{H}), 7.03(\mathrm{tdd}, J=7.5,5.3,1.8 \mathrm{~Hz}, 1 \mathrm{H}), 6.85-6.78(\mathrm{~m}, 2 \mathrm{H}),[6.25$ $-6.17(\mathrm{~m}$, minor diastereoisomer $)+6.10-6.04(\mathrm{~m}$, major diastereoisomer $), 1 \mathrm{H}], 4.16-4.11(\mathrm{~m}, 0.5 \mathrm{H})$, $3.77-3.68(\mathrm{~m}, 3.5 \mathrm{H}), 2.76-2.46(\mathrm{~m}, 1 \mathrm{H}), 2.26(\mathrm{ddt}, J=17.7,9.7,5.3 \mathrm{~Hz}, 1 \mathrm{H})$.

${ }^{13} \mathrm{C}$ NMR $\left(151 \mathrm{MHz}, \mathrm{CDCl}_{3}\right)\left\{{ }^{19} \mathrm{~F}\right\}$ : (mixture of diastereoisomers and regioisomer) $\delta 165.5,165.3$, $159.8,159.7,145.3,145.1,141.4,141.3,135.4,134.9,132.7,132.6,131.8,131.7,131.6,131.5,131.1$, 131.0, 130.9, 129.4, 128.7, 128.6, 128.5, 128.4, 128.3, 128.2, 128.2, 128.2, 128.2, 128.1, 128.0, 127.9, $127.8,127.8,127.4,127.3,127.2,126.9,125.7,125.6,124.1,123.0,123.0,114.1,114.0,94.1,93.9,89.0$, $88.9,85.0,84.8,75.7,75.6,55.3,55.2,44.6,44.1,35.5,35.1$.

${ }^{19} \mathrm{~F}$ NMR $\left(282 \mathrm{MHz}, \mathrm{CDCl}_{3}\right.$ ) (mixture of diastereoisomers and regioisomer) $\delta-62.24,-62.34,-62.52,-$ 62.58 .

HRMS (ESI) m/z: [M+Na $]^{+}$Calcd for $\mathrm{C}_{32} \mathrm{H}_{24} \mathrm{~F}_{3} \mathrm{INaO}_{3}$ 663.0620; Found: 663.0614 .

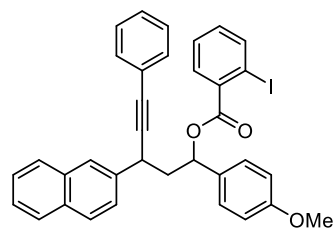

\section{1-(4-Methoxyphenyl)-3-(naphthalen-2-yl)-5-phenylpent-4-yn-1-yl 2-iodobenzoate (3w)}

Purified by flash silica column chromatography (pentane/ethyl acetate $=50: 1$ ) as inseparable mixture of diastereoisomers $(\mathrm{dr}=1.5: 1)$ and regioisomeric $(6: 1 \mathrm{rr})$

Colorless oil (71.0 mg, $57 \%$ yield)

${ }^{1} \mathrm{H}$ NMR (300 MHz, $\mathrm{CDCl}_{3}$ ): (mixture of diastereoisomers and regioisomer) $\delta 7.98-7.87(\mathrm{~m}, 1 \mathrm{H})$, $7.84-7.60(\mathrm{~m}, 5 \mathrm{H}), 7.48-7.21(\mathrm{~m}, 11 \mathrm{H}), 7.10-6.97(\mathrm{~m}, 1 \mathrm{H}), 6.82(\mathrm{ddq}, J=12.1,7.5,2.5 \mathrm{~Hz}, 2 \mathrm{H})$, $[6.25-6.19(\mathrm{~m}$, major diastereoisomer $)+6.14-6.09(\mathrm{~m}$, minor diastereoisomer $), 1 \mathrm{H}],[4.25-4.20(\mathrm{~m})$ $+3.91-3.77(\mathrm{~m}) 1 \mathrm{H}], 3.74-3.66(\mathrm{~m}, 3 \mathrm{H}), 2.79-2.60(\mathrm{~m}, 1 \mathrm{H}), 2.47-2.28(\mathrm{~m}, 1 \mathrm{H})$.

${ }^{13} \mathrm{C}$ NMR (76 MHz, $\mathrm{CDCl}_{3}$ ): (mixture of diastereoisomers and regioisomer) $\delta 165.6,165.4,159.7$, 159.6, 158.7, 141.5, 141.4, 141.3, 138.6, 138.4, 137.3, 135.4, 135.0, 134.1, 133.6, 133.2, 132.6, 131.9, $131.8,131.7,131.4,131.2,131.1,128.9,128.7,128.6,128.5,128.4,128.3,128.2,128.1,127.9,127.8$, 
$127.7,126.4,126.2,126.1,126.0,125.8,125.7,124.4,123.4,114.2,114.1,114.0,94.0,94.2,90.2,90.1$, $84.6,84.4,75.9,55.3,44.6,44.2,35.7,35.4$.

HRMS (ESI) m/z: [M+Na] $]^{+}$Calcd for $\mathrm{C}_{35} \mathrm{H}_{27} \mathrm{INaO}_{3}$ 645.0903; Found: 645.0901 .

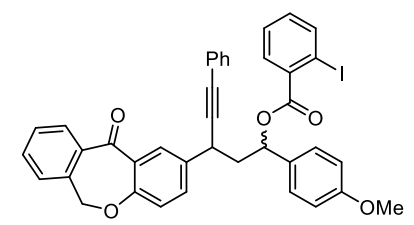

1-(4-Methoxyphenyl)-3-(11-oxo-6,11-dihydrodibenzo[b,e]oxepin-2-yl)-5-phenylpent-4-yn-1-yl 2iodobenzoate $(3 \mathbf{x})$

Purified by flash silica column chromatography (pentane/ethyl acetate $=20: 1$ ) as inseparable diastereoisomer $(1.6: 1 \mathrm{dr})$ and regioisomer $(1.1: 1 \mathrm{rr})$

Yellow oil (83.1 mg, 59\% yield)

${ }^{1} \mathrm{H}$ NMR (300 MHz, $\left.\mathrm{CDCl}_{3}\right)$ : (mixture of diastereoisomers and regioisomer) $\delta 8.34-8.05(\mathrm{~m}, 1 \mathrm{H})$, $7.93-7.62(\mathrm{~m}, 3 \mathrm{H}), 7.60-7.20(\mathrm{~m}, 12 \mathrm{H}), 7.05-6.92(\mathrm{~m}, 2 \mathrm{H}), 6.85-6.72(\mathrm{~m}, 2 \mathrm{H}), 6.29-5.92$ $(\mathrm{m}, 1 \mathrm{H}), 5.11-5.03(\mathrm{~m}, 2 \mathrm{H}), 4.10(\mathrm{dt}, J=10.8,5.4 \mathrm{~Hz}, 0.5 \mathrm{H}), 3.71-3.68(\mathrm{~m}, 3.5 \mathrm{H}), 2.75-2.45$ $(\mathrm{m}, 1 \mathrm{H}), 2.42-2.13(\mathrm{~m}, 1 \mathrm{H})$.

${ }^{13} \mathrm{C}$ NMR (76 MHz, $\mathrm{CDCl}_{3}$ ): (mixture of diastereoisomers and regioisomer) $\delta 190.9,165.6,165.4$, $161.1,160.4,159.7,159.6,158.6,141.5,141.4,140.5,135.6,135.4,134.9,134.8,134.6,134.5,134.4$, 134.2, 133.9, 133.4, 132.8, 132.6, 131.9, 131.8, 131.7, 131.3, 131.2, 131.0, 130.6, 130.5, 130.4, 130.3, $129.5,129.3,129.2,128.6,128.5,128.4,128.3,128.2,128.1,127.9,127.8,125.2,123.4,123.3,121.3$, $121.1,114.3,114.2,114.1,114.0,94.2,90.5,89.7,89.6,84.6,84.0,75.9,75.8,75.6,73.6,55.3,44.7$, $44.4,43.9,34.7,34.4$

HRMS (ESI) m/z: [M+Na] $]^{+}$Calcd for $\mathrm{C}_{39} \mathrm{H}_{29} \mathrm{INaO}_{5}$ 727.0957; Found: 727.0955 .

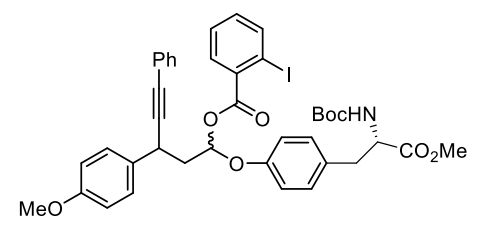

1-(4-((S)-2-((tert-Butoxycarbonyl)amino)-3-methoxy-3-oxopropyl)phenoxy)-3-(4-methoxyphenyl)5-phenylpent-4-yn-1-yl 2-iodobenzoate (3y)

Purified by flash silica column chromatography (pentane/ethyl acetate $=5: 1$ ) as inseparable diastereoisomer $(\mathrm{dr}=1.1: 1)$

Yellow oil (77.3 mg, 49\% yield) 
${ }^{1} \mathrm{H}$ NMR (300 MHz, $\left.\mathrm{CDCl}_{3}\right)$ : (mixture of diastereoisomers) $\delta 7.98-7.84(\mathrm{~m}, 2 \mathrm{H}), 7.67-7.62(\mathrm{~m}, 1 \mathrm{H})$, $7.37-7.20(\mathrm{~m}, 6 \mathrm{H}), 7.12-6.78(\mathrm{~m}, 8 \mathrm{H}), 4.91(\mathrm{~d}, J=7.3 \mathrm{~Hz}, 1 \mathrm{H}), 4.46(\mathrm{~s}, 1 \mathrm{H}), 4.12(\mathrm{td}, J=8.0,4.9 \mathrm{~Hz}$, 1H), $3.72(\mathrm{~s}, 3 \mathrm{H}), 3.64-3.54(\mathrm{~m}, 3 \mathrm{H}), 2.94(\mathrm{t}, J=5.5 \mathrm{~Hz}, 2 \mathrm{H}), 2.67-2.34(\mathrm{~m}, 2 \mathrm{H}), 1.33(\mathrm{t}, J=3.1 \mathrm{~Hz}$, $9 \mathrm{H})$.

${ }^{13} \mathrm{C}$ NMR (76 MHz, $\mathrm{CDCl}_{3}$ ): (mixture of diastereoisomers) $\delta 172.3,169.8,165.1,158.8,158.7,155.4$, $155.2,154.9,141.8,141.5,134.2,133.7,133.3,132.9,132.8,132.4,131.9,131.8,131.7,131.6,131.2$, $130.6,130.5,128.6,128.5,128.4,128.3,128.2,128.1,128.0,127.9,127.8,123.4,123.3,117.4,117.1$, $115.5,114.2,114.1,113.8,96.5,95.5,94.6,94.2,90.5,89.7,84.4,83.6,80.1,55.3,54.5,52.3,42.8,42.4$, $37.6,33.4,33.2,28.3$.

HRMS (ESI) m/z: [M+Na $]^{+}$Calcd for $\mathrm{C}_{40} \mathrm{H}_{40} \mathrm{INNaO}_{8}$ 812.1696; Found: 812.1694.

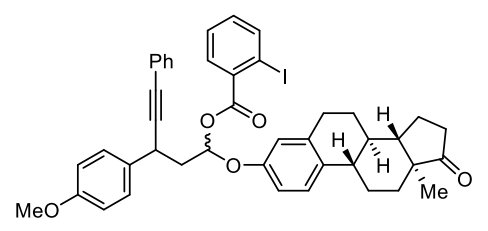

3-(4-Methoxyphenyl)-1-((13-methyl-17-oxo-7,8,9,11,12,13,14,15,16,17-decahydro-6H-

cyclopenta[a]phenanthren-3-yl)oxy)-5-phenylpent-4-yn-1-yl 2-iodobenzoate (3z)

Purified by flash silica column chromatography (pentane/ethyl acetate $=10: 1$ ) as inseparable diastereoisomer $(\mathrm{dr}=1.1: 1)$

Yellow oil (81.1 mg, 53\% yield)

${ }^{1} \mathrm{H}$ NMR (300 MHz, $\left.\mathrm{CDCl}_{3}\right)$ : (mixture of diastereoisomers) $\delta 8.06-7.80(\mathrm{~m}, 2 \mathrm{H}), 7.52-7.26(\mathrm{~m}, 6 \mathrm{H})$, $7.19(\mathrm{t}, J=3.1 \mathrm{~Hz}, 2 \mathrm{H}), 7.10-6.96(\mathrm{~m}, 2 \mathrm{H}), 6.81-6.62(\mathrm{~m}, 4 \mathrm{H}), 4.11(\mathrm{t}, J=7.5 \mathrm{~Hz}, 1 \mathrm{H}), 3.71(\mathrm{~s}, 3 \mathrm{H})$, $2.75(\mathrm{dd}, J=9.7,5.1 \mathrm{~Hz}, 2 \mathrm{H}), 2.48-1.89(\mathrm{~m}, 9 \mathrm{H}), 1.57-1.31(\mathrm{~m}, 7 \mathrm{H}), 0.81(\mathrm{~s}, 3 \mathrm{H})$.

${ }^{13} \mathrm{C}$ NMR (76 MHz, $\mathrm{CDCl}_{3}$ ): (mixture of diastereoisomers) $\delta 221.1,165.0,158.8,158.7,154.5,153.9$, $153.8,141.5,141.4,138.2,138.1,134.7,134.5,132.9,132.5,131.9,131.8,131.7,131.6,131.3,131.2$, $130.2,128.6,128.5,128.4,128.2,128.1,128.0,127.9,126.6,126.5,123.4,123.3,117.5,117.4,117.1$, 117.0, 114.8, 114.7, 114.4, 114.2, 114.1, 96.8, 96.7, 95.7, 95.6, 94.4, 94.3, 90.6, 89.8, 84.4, 83.5, 55.3, $50.4,48.0,44.0,42.7,42.4,38.2,35.9,33.4,33.2,31.6,29.6,26.5,25.8,21.6,13.9$.

HRMS (ESI) m/z: [M+Na] $]^{+}$Calcd for $\mathrm{C}_{43} \mathrm{H}_{41} \mathrm{INaO}_{5}$ 787.1896; Found: 787.1897.

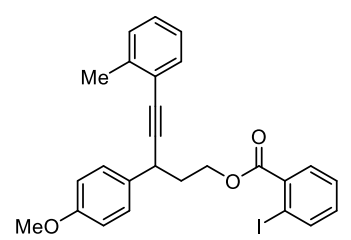


3-(4-Methoxyphenyl)-5-(o-tolyl)pent-4-yn-1-yl 2-iodobenzoate (3aa)

Purified by flash silica column chromatography (pentane/ethyl acetate $=40: 1$ )

Colorless oil (57.2 mg, $56 \%$ yield)

${ }^{1} \mathrm{H}$ NMR $\left(300 \mathrm{MHz}, \mathrm{CDCl}_{3}\right): \delta 7.93-7.88(\mathrm{~m}, 1 \mathrm{H}), 7.68(\mathrm{dd}, J=7.8,1.7 \mathrm{~Hz}, 1 \mathrm{H}), 7.37-7.28(\mathrm{~m}, 5 \mathrm{H})$, $7.08(\mathrm{ddt}, J=15.3,4.2,2.6 \mathrm{~Hz}, 4 \mathrm{H}), 6.83(\mathrm{~d}, J=1.9 \mathrm{~Hz}, 1 \mathrm{H}), 4.46(\mathrm{ddq}, J=28.6,11.3,6.2 \mathrm{~Hz}, 2 \mathrm{H}), 4.08$ $(\mathrm{t}, J=7.4 \mathrm{~Hz}, 1 \mathrm{H}), 3.72(\mathrm{~s}, 3 \mathrm{H}), 2.36(\mathrm{~s}, 3 \mathrm{H}), 2.22(\mathrm{q}, J=4.3,2.9 \mathrm{~Hz}, 2 \mathrm{H})$.

${ }^{13} \mathrm{C} \mathrm{NMR}\left(76 \mathrm{MHz}, \mathrm{CDCl}_{3}\right) \delta 164.4,156.5,139.2,137.8,133.2,131.1,130.6,129.9,128.9,127.3,126.4$, $125.9,125.8,123.4,12119,112.0,92.3,91.9,80.7,61.6,53.2,35.3,32.6,18.9$.

HRMS (ESI) m/z: [M+Na] $]^{+}$Calcd for $\mathrm{C}_{26} \mathrm{H}_{23} \mathrm{INaO}_{3}$ 533.0590; Found: 533.0585 .

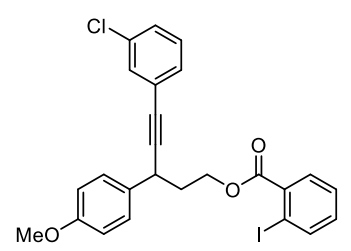

\section{5-(3-Chlorophenyl)-3-(4-methoxyphenyl)pent-4-yn-1-yl 2-iodobenzoate (3ab)}

Purified by flash silica column chromatography (pentane/ethyl acetate $=40: 1$ )

Colorless oil (65.8 mg, $62 \%$ yield)

${ }^{1} \mathrm{H}$ NMR $\left(300 \mathrm{MHz}, \mathrm{CDCl}_{3}\right): \delta 7.92(\mathrm{dd}, J=8.0,1.3 \mathrm{~Hz}, 1 \mathrm{H}), 7.69(\mathrm{dd}, J=7.8,1.7 \mathrm{~Hz}, 1 \mathrm{H}), 7.32-7.08$ $(\mathrm{m}, 8 \mathrm{H}), 6.85-6.76(\mathrm{~m}, 2 \mathrm{H}), 4.53-4.32(\mathrm{~m}, 2 \mathrm{H}), 4.02(\mathrm{t}, J=7.4 \mathrm{~Hz}, 1 \mathrm{H}), 3.73(\mathrm{~s}, 3 \mathrm{H}), 2.29-2.12(\mathrm{~m}$, $2 \mathrm{H})$.

${ }^{13} \mathrm{C} \mathrm{NMR}\left(76 \mathrm{MHz}, \mathrm{CDCl}_{3}\right): \delta$ 166.5, 158.7, 142.2, 141.3, 135.2, 134.0, 132.4, 131.6, 131.1, 129.9, 129.5, $128.5,128.3,127.9,125.1,114.2,94.1,91.9,82.5,63.5,55.3,37.0,34.5$.

HRMS (ESI) m/z: [M+Na $]^{+}$Calcd for $\mathrm{C}_{25} \mathrm{H}_{20} \mathrm{ClINaO}_{3}$ 553.0043; Found: 553.0038 .

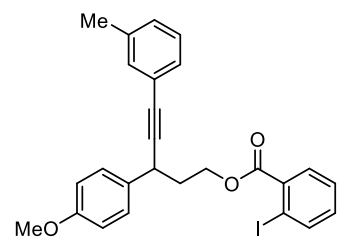

3-(4-Methoxyphenyl)-5-(m-tolyl)pent-4-yn-1-yl 2-iodobenzoate (3ac)

Purified by flash silica column chromatography (pentane/ethyl acetate $=40: 1$ )

Colorless oil (69.4 mg, 68\% yield) 
${ }^{1} \mathrm{H}$ NMR $\left(300 \mathrm{MHz}, \mathrm{CDCl}_{3}\right): \delta 8.08-7.97(\mathrm{~m}, 1 \mathrm{H}), 7.80(\mathrm{dd}, J=7.7,1.8 \mathrm{~Hz}, 1 \mathrm{H}), 7.44-7.34(\mathrm{~m}, 3 \mathrm{H})$, $7.31-7.07(\mathrm{~m}, 5 \mathrm{H}), 6.98-6.85(\mathrm{~m}, 2 \mathrm{H}), 4.72-4.37(\mathrm{~m}, 2 \mathrm{H}), 4.14(\mathrm{t}, J=7.4 \mathrm{~Hz}, 1 \mathrm{H}), 3.83(\mathrm{~s}, 3 \mathrm{H}), 2.40$ $-2.25(\mathrm{~m}, 5 \mathrm{H})$.

${ }^{13} \mathrm{C} \mathrm{NMR}\left(76 \mathrm{MHz}, \mathrm{CDCl}_{3}\right): \delta 166.5,158.6,141.3,137.9,135.2,133.1,132.7,132.3,131.1,128.9,128.8$, $128.5,128.2,127.9,123.2,114.1,94.1,90.0,84.1,63.7,55.4,37.3,34.6,21.3$.

HRMS (ESI) m/z: [M+Na] $]^{+}$Calcd for $\mathrm{C}_{26} \mathrm{H}_{23} \mathrm{INaO}_{3}$ 533.0590; Found: 533.0582.

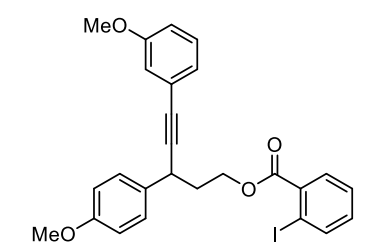

5-(3-Methoxyphenyl)-3-(4-methoxyphenyl)pent-4-yn-1-yl 2-iodobenzoate (3ad)

Purified by flash silica column chromatography (pentane/ethyl acetate $=40: 1$ )

Colorless oil (62.1 mg, 59\% yield)

${ }^{1} \mathrm{H}$ NMR $\left(300 \mathrm{MHz}, \mathrm{CDCl}_{3}\right): \delta 7.95-7.85(\mathrm{~m}, 1 \mathrm{H}), 7.70(\mathrm{dd}, J=7.8,1.8 \mathrm{~Hz}, 1 \mathrm{H}), 7.36-7.26(\mathrm{~m}, 3 \mathrm{H})$, $7.16-7.06(\mathrm{~m}, 2 \mathrm{H}), 7.00-6.77(\mathrm{~m}, 5 \mathrm{H}), 4.52-4.33(\mathrm{~m}, 2 \mathrm{H}), 4.04(\mathrm{t}, J=7.4 \mathrm{~Hz}, 1 \mathrm{H}), 3.73(\mathrm{~s}, 3 \mathrm{H}), 3.72$ (s, 3H), $2.29-2.12(\mathrm{~m}, 2 \mathrm{H})$.

${ }^{13} \mathrm{C}$ NMR $\left(151 \mathrm{MHz}, \mathrm{CDCl}_{3}\right): \delta 166.5,159.3,158.7,141.3,135.3,132.9,132.6,131.0,129.3,128.5$, $127.9,124.4,124.3,116.5,114.6,114.1,93.9,90.3,83.7,63.6,55.3,55.2,37.2,34.5$.

HRMS (ESI) m/z: [M+Na] $]^{+}$Calcd for $\mathrm{C}_{26} \mathrm{H}_{23} \mathrm{INaO}_{4}$ 549.0539; Found: 549.0532.

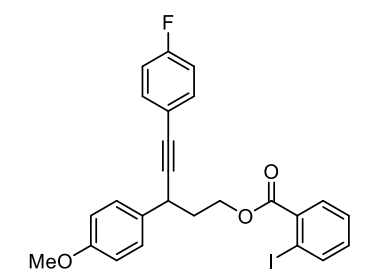

5-(4-Fluorophenyl)-3-(4-methoxyphenyl)pent-4-yn-1-yl 2-iodobenzoate (3ae)

Purified by flash silica column chromatography (pentane/ethyl acetate $=40: 1$ )

Colorless oil (58.6 mg, $57 \%$ yield)

${ }^{1} \mathrm{H}$ NMR $\left(300 \mathrm{MHz}, \mathrm{CDCl}_{3}\right): \delta 8.03-7.97(\mathrm{~m}, 1 \mathrm{H}), 7.79(\mathrm{dd}, J=7.7,1.8 \mathrm{~Hz}, 1 \mathrm{H}), 7.48-7.33(\mathrm{~m}, 5 \mathrm{H})$, $7.20-7.14(\mathrm{~m}, 1 \mathrm{H}), 7.05-6.96(\mathrm{~m}, 2 \mathrm{H}), 6.96-6.86(\mathrm{~m}, 2 \mathrm{H}), 4.54(\mathrm{ddt}, J=22.9,11.4,6.3 \mathrm{~Hz}, 2 \mathrm{H})$, $4.12(\mathrm{t}, J=7.4 \mathrm{~Hz}, 1 \mathrm{H}), 3.82(\mathrm{~s}, 3 \mathrm{H}), 2.31(\mathrm{dt}, J=7.9,6.4 \mathrm{~Hz}, 2 \mathrm{H})$.

${ }^{13} \mathrm{C}$ NMR $\left(151 \mathrm{MHz}, \mathrm{CDCl}_{3}\right)\left\{{ }^{19} \mathrm{~F}\right\}: \delta 166.5,163.1,161.5,158.7,141.3,135.2,133.5,133.5,132.9$, 132.6, 131.0, 128.4, 127.9, 115.5, 115.4, 114.1, 94.0, 90.1, 82.8, 63.6, 55.3, 37.1, 34.5. 
${ }^{19} \mathrm{~F}$ NMR (282 MHz, $\left.\mathrm{CDCl}_{3}\right): \delta-111.54$.

HRMS (ESI) m/z: [M+Na $]^{+}$Calcd for $\mathrm{C}_{25} \mathrm{H}_{20} \mathrm{FINaO}_{3}$ 537.0339; Found: 537.0334 .

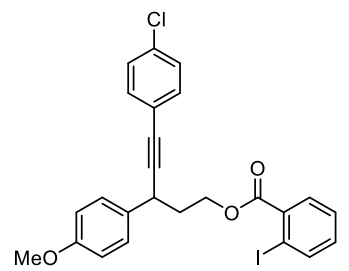

\section{5-(4-Chlorophenyl)-3-(4-methoxyphenyl)pent-4-yn-1-yl 2-iodobenzoate (3af)}

Purified by flash silica column chromatography (pentane/ethyl acetate $=40: 1$ )

Colorless oil (58.3 mg, 55\% yield)

${ }^{1} \mathrm{H} \mathrm{NMR}\left(300 \mathrm{MHz}, \mathrm{CDCl}_{3}\right): \delta 7.92(\mathrm{dd}, J=7.9,1.2 \mathrm{~Hz}, 1 \mathrm{H}), 7.73-7.63(\mathrm{~m}, 1 \mathrm{H}), 7.33-7.23(\mathrm{~m}, 5 \mathrm{H})$, $7.19(\mathrm{~d}, J=3.7 \mathrm{~Hz}, 2 \mathrm{H}), 7.08(\mathrm{td}, J=7.6,1.7 \mathrm{~Hz}, 1 \mathrm{H}), 6.87-6.78(\mathrm{~m}, 2 \mathrm{H}), 4.55-4.30(\mathrm{~m}, 2 \mathrm{H}), 4.02(\mathrm{t}$, $J=7.4 \mathrm{~Hz}, 1 \mathrm{H}), 3.73(\mathrm{~s}, 3 \mathrm{H}), 2.29-2.10(\mathrm{~m}, 2 \mathrm{H})$.

${ }^{13} \mathrm{C}$ NMR $\left(76 \mathrm{MHz}, \mathrm{CDCl}_{3}\right): \delta 166.5,158.7,141.3,135.2,133.9,132.9,132.8,132.7,131.1,128.6,128.5$, $127.9,121.9,114.1,94.0,91.5,82.8,63.6,55.3,37.1,34.5$.

HRMS (ESI) m/z: [M+Na] $]^{+}$Calcd for $\mathrm{C}_{25} \mathrm{H}_{20} \mathrm{ClINaO}_{3}$ 553.0043; Found: 553.0038 .

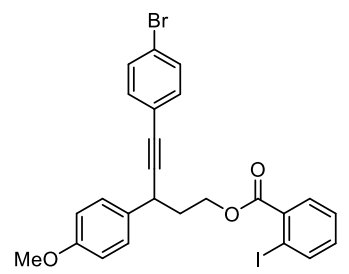

\section{5-(4-Bromophenyl)-3-(4-methoxyphenyl)pent-4-yn-1-yl 2-iodobenzoate (3ag)}

Purified by flash silica column chromatography (pentane/ethyl acetate $=40: 1$ )

Colorless oil (70.2 mg, $61 \%$ yield)

${ }^{1} \mathrm{H}$ NMR $\left(300 \mathrm{MHz}, \mathrm{CDCl}_{3}\right): \delta 7.96-7.88(\mathrm{~m}, 1 \mathrm{H}), 7.68(\mathrm{dd}, J=7.8,1.8 \mathrm{~Hz}, 1 \mathrm{H}), 7.36-7.16(\mathrm{~m}, 7 \mathrm{H})$, $7.09-7.01(\mathrm{~m}, 1 \mathrm{H}), 6.86-6.78(\mathrm{~m}, 2 \mathrm{H}), 4.57-4.28(\mathrm{~m}, 2 \mathrm{H}), 4.07-3.94(\mathrm{~m}, 1 \mathrm{H}), 3.72(\mathrm{~s}, 3 \mathrm{H}), 2.20(\mathrm{q}$, $J=6.6 \mathrm{~Hz}, 2 \mathrm{H})$.

${ }^{13} \mathrm{CNMR}\left(76 \mathrm{MHz}, \mathrm{CDCl}_{3}\right): \delta 166.5,158.7,141.3,135.2,133.2,132.7,131.5,131.1,128.5,127.9,122.4$, 122.1, 114.2, 94.1, 91.7, 82.8, 63.6, 55.4, 37.0, 34.6.

HRMS (ESI) m/z: [M+Na] $]^{+}$Calcd for $\mathrm{C}_{26} \mathrm{H}_{25} \mathrm{ClNaO}_{3}$ 596.9538; Found: 596.9534.

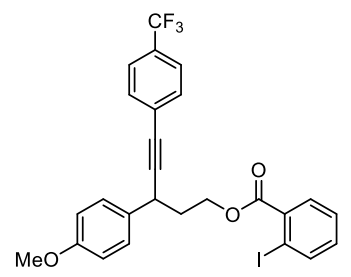


Purified by flash silica column chromatography (pentane/ethyl acetate $=40: 1$ )

Colorless oil (59.8 mg, 53\% yield)

${ }^{1} \mathrm{H}$ NMR $\left(300 \mathrm{MHz}, \mathrm{CDCl}_{3}\right): \delta 7.79(\mathrm{dt}, J=8.0,1.2 \mathrm{~Hz}, 1 \mathrm{H}), 7.57(\mathrm{dt}, J=7.7,1.4 \mathrm{~Hz}, 1 \mathrm{H}), 7.33(\mathrm{~d}, J=$ $18.7 \mathrm{~Hz}, 4 \mathrm{H}), 7.24-7.11(\mathrm{~m}, 3 \mathrm{H}), 6.94(\mathrm{tt}, J=7.6,1.5 \mathrm{~Hz}, 1 \mathrm{H}), 6.78-6.62(\mathrm{~m}, 2 \mathrm{H}), 4.33(\mathrm{ddt}, J=21.9$, $11.2,6.0 \mathrm{~Hz}, 2 \mathrm{H}), 3.93(\mathrm{t}, J=7.4 \mathrm{~Hz}, 1 \mathrm{H}), 3.60(\mathrm{~d}, J=1.1 \mathrm{~Hz}, 3 \mathrm{H}), 2.11(\mathrm{q}, J=6.6 \mathrm{~Hz}, 2 \mathrm{H})$.

${ }^{13} \mathrm{C}$ NMR $\left(126 \mathrm{MHz}, \mathrm{CDCl}_{3}\right)\left\{{ }^{19} \mathrm{~F}\right\}: \delta 166.5,158.8,141.3,135.2,132.7,132.5,131.9,131.8,131.0$, $129.7,128.5,127.9,127.2,126.0,125.2,125.1,123.9,114.2,94.0,93.3,82.6,63.5,55.3,36.9,34.6$. ${ }^{19} \mathrm{~F}$ NMR (282 MHz, $\left.\mathrm{CDCl}_{3}\right): \delta-62.67$.

HRMS (ESI) m/z: [M+Na] $]^{+}$Calcd for $\mathrm{C}_{26} \mathrm{H}_{20} \mathrm{~F}_{3} \mathrm{INaO}_{3}$ 587.0307; Found: 587.0302.

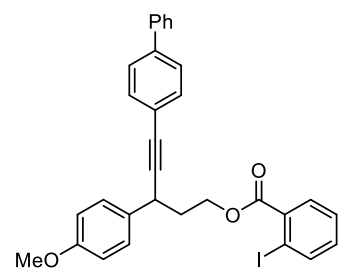

\section{5-([1,1'-Biphenyl]-4-yl)-3-(4-methoxyphenyl)pent-4-yn-1-yl 2-iodobenzoate (3ai)}

Purified by flash silica column chromatography (pentane/ethyl acetate $=40: 1$ )

Colorless oil (72.1 mg, 63\% yield)

${ }^{1} \mathrm{H}$ NMR $\left(300 \mathrm{MHz}, \mathrm{CDCl}_{3}\right): \delta 8.02(\mathrm{~d}, J=7.9 \mathrm{~Hz}, 1 \mathrm{H}), 7.83-7.76(\mathrm{~m}, 1 \mathrm{H}), 7.61-7.36(\mathrm{~m}, 12 \mathrm{H}), 7.17$ $(\mathrm{dd}, J=8.4,6.8 \mathrm{~Hz}, 1 \mathrm{H}), 6.92(\mathrm{~d}, J=8.8 \mathrm{~Hz}, 2 \mathrm{H}), 4.57(\mathrm{dp}, J=22.0,6.0,5.5 \mathrm{~Hz}, 2 \mathrm{H}), 4.16(\mathrm{t}, J=7.3$ $\mathrm{Hz}, 1 \mathrm{H}), 3.83(\mathrm{~s}, 3 \mathrm{H}), 2.33(\mathrm{q}, J=6.5 \mathrm{~Hz}, 2 \mathrm{H})$.

${ }^{13} \mathrm{C}$ NMR $\left(126 \mathrm{MHz}, \mathrm{CDCl}_{3}\right): \delta 166.5,158.7,141.3,140.7,140.4,135.3,133.0,132.6,132.3,132.1$, 131.1, 128.8, 128.5, 127.9, 127.6, 127.0, 126.9, 122.3, 114.1, 94.0, 91.1, 83.7, 63.7, 55.3, 37.2, 34.6.

HRMS (ESI) m/z: [M+Na]+ Calcd for $\mathrm{C}_{31} \mathrm{H}_{25} \mathrm{INaO}_{3}$ 595.0746; Found: 595.0744.

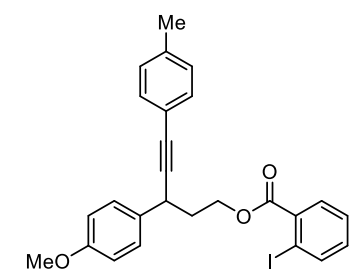

3-(4-Methoxyphenyl)-5-(p-tolyl)pent-4-yn-1-yl 2-iodobenzoate (3aj)

Purified by flash silica column chromatography (pentane/ethyl acetate $=40: 1$ )

Colorless oil (60.2 mg, $59 \%$ yield) 
${ }^{1} \mathrm{H} \mathrm{NMR}\left(300 \mathrm{MHz}, \mathrm{CDCl}_{3}\right): \delta 7.91(\mathrm{dd}, J=7.9,1.2 \mathrm{~Hz}, 1 \mathrm{H}), 7.69(\mathrm{dd}, J=7.8,1.7 \mathrm{~Hz}, 1 \mathrm{H}), 7.34-7.22$ (m, 5H), $7.10-7.00(\mathrm{~m}, 3 \mathrm{H}), 6.85-6.76(\mathrm{~m}, 2 \mathrm{H}), 4.59-4.28(\mathrm{~m}, 2 \mathrm{H}), 4.02(\mathrm{t}, J=7.4 \mathrm{~Hz}, 1 \mathrm{H}), 3.72(\mathrm{~s}$, 3H), $2.26(\mathrm{~s}, 3 \mathrm{H}), 2.19$ (dd, $J=8.1,6.1 \mathrm{~Hz}, 2 \mathrm{H})$.

${ }^{13} \mathrm{C} \mathrm{NMR}\left(76 \mathrm{MHz}, \mathrm{CDCl}_{3}\right): \delta 166.5,158.6,141.3,137.9,135.3,133.2,132.7,131.6,131.1,129.0,128.5$, $127.9,120.3,114.1,94.1,89.6,83.9,63.7,55.3,37.3,34.5,21.5$.

HRMS (ESI) m/z: [M+Na] $]^{+}$Calcd for $\mathrm{C}_{26} \mathrm{H}_{23} \mathrm{INaO}_{3}$ 533.0590; Found: 533.0583.

\section{Large-scale preparation of $3 a$ and its derivatization:}

\section{(a) Large-scale preparation of 3a}

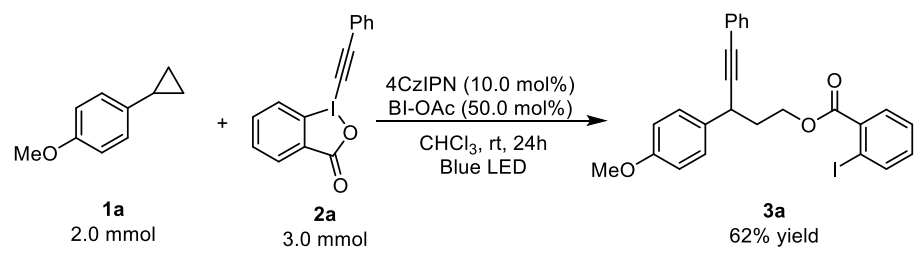

To an oven-dried $50 \mathrm{~mL}$ Schlenk tube equipped with a stir bar, aryl cyclopropane 1a (296 mg, $2.0 \mathrm{mmol}$, 1.0 equiv), EBX 2a (1.04 g, 3.0 mmol, 1.5 equiv), photocatalyst 4CzIPN (157.6 mg, 10.0 mol\%), BIOAc (306 mg, 0.5 equiv). Then the reaction tube was evacuated and backfilled with argon for two times. Subsequently, anhydrous $\mathrm{CHCl}_{3}(10.0 \mathrm{~mL}, 0.2 \mathrm{M})$ were added. The resulting mixture was irradiated under blue LEDs at room temperature for $24 \mathrm{~h}$. The residue was purified by silica gel chromatography to afford the desired product $\mathbf{3 a}(615.0 \mathrm{mg}, 62 \%$ yield $)$.

\section{(b) Saponification of 3a}

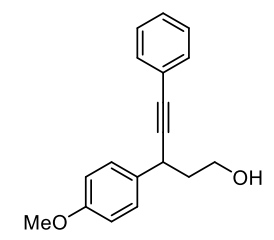

\section{4-Hydroxy-2-(4-methoxyphenyl)-1-phenylbutan-1-one (4a)}

A solution of $\mathbf{3 a}(49.6 \mathrm{mg}, 0.10 \mathrm{mmol})$ in THF $(1.0 \mathrm{~mL})$ was added $\mathrm{NaOH}$ aq $(1 \mathrm{~mL}, 1 \mathrm{M})$ solution. The reaction mixture was stirred at $80{ }^{\circ} \mathrm{C}$ for $6 \mathrm{~h}$. After completion, the mixture was extracted with ethyl acetate $(3 \times 3 \mathrm{~mL})$. The organic layer was dried by $\mathrm{Na}_{2} \mathrm{SO}_{4}$ and evaporated under reduced pressure. Then the residue was purified by purified by column chromatography on silica gel (pentane/ethyl acetate $=5: 1$ ) to afford the desired product $4 \mathbf{a}$ as colorless oil $(21.8 \mathrm{mg}, 82 \%$ yield).

${ }^{1} \mathrm{H}$ NMR $\left(300 \mathrm{MHz}, \mathrm{CDCl}_{3}\right): \delta 7.56-7.23(\mathrm{~m}, 7 \mathrm{H}), 7.00-6.82(\mathrm{~m}, 2 \mathrm{H}), 4.06(\mathrm{t}, J=7.5 \mathrm{~Hz}, 1 \mathrm{H}), 3.93$ (dt, $J=10.9,6.4 \mathrm{~Hz}, 1 \mathrm{H}), 3.83(\mathrm{~s}, 4 \mathrm{H}), 2.08(\mathrm{dt}, J=7.5,5.9 \mathrm{~Hz}, 2 \mathrm{H})$. 
${ }^{13} \mathrm{C}$ NMR $\left(76 \mathrm{MHz}, \mathrm{CDCl}_{3}\right): \delta 158.1,133.6,131.7,128.5,128.3,127.9,123.5,114.0,91.1,83.6,60.8$, $55.3,41.2,34.2$.

HRMS (ESI) m/z: [M+Na] $]^{+}$Calcd for $\mathrm{C}_{18} \mathrm{H}_{18} \mathrm{NaO}_{2}$ 289.1204; Found: 289.1199.

(c) Hydration of 3a

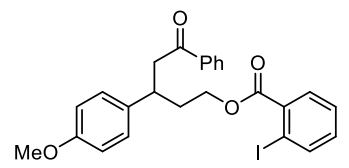

3-(4-Methoxyphenyl)-5-oxo-5-phenylpentyl 2-iodobenzoate (4b)

To a solution of $3 \mathbf{a}$ ( $49.6 \mathrm{mg}, 0.10$ mmol, 1.0 equiv.) in $\mathrm{CF}_{3} \mathrm{CH}_{2} \mathrm{OH}(0.5 \mathrm{~mL})$ were added $\mathrm{CF}_{3} \mathrm{SO}_{3} \mathrm{H}(3.0$ $\mathrm{mg}, 0.02 \mathrm{mmol}, 0.2$ equiv) and $\mathrm{H}_{2} \mathrm{O}(3.6 \mathrm{mg}, 0.20 \mathrm{mmol}, 2.0$ equiv). And the reaction mixture was stirred at $80{ }^{\circ} \mathrm{C}$ for $16 \mathrm{~h}$. After evaporation under reduced pressure, the residue was purified with column chromatography on silica gel to yield the product $\mathbf{4 b}$ as a colorless oil ( $26.2 \mathrm{mg}, 51 \%$ yield).

${ }^{1} \mathrm{H}$ NMR $\left(300 \mathrm{MHz}, \mathrm{CDCl}_{3}\right): \delta 7.96-7.80(\mathrm{~m}, 3 \mathrm{H}), 7.62(\mathrm{dd}, J=7.8,1.7 \mathrm{~Hz}, 1 \mathrm{H}), 7.47(\mathrm{t}, J=7.4 \mathrm{~Hz}$, 1H), $7.39-7.26(\mathrm{~m}, 3 \mathrm{H}), 7.15-7.04(\mathrm{~m}, 3 \mathrm{H}), 6.76(\mathrm{~d}, J=8.8 \mathrm{~Hz}, 2 \mathrm{H}), 4.29-3.98(\mathrm{~m}, 2 \mathrm{H}), 3.48(\mathrm{~s}$, $1 \mathrm{H}), 3.29-3.16(\mathrm{~m}, 2 \mathrm{H}), 2.26-1.90(\mathrm{~m}, 2 \mathrm{H})$.

${ }^{13} \mathrm{C}$ NMR (151 MHz, $\left.\mathrm{CDCl}_{3}\right): \delta 198.6,166.3,158.3,141.3,137.0,135.4,135.1,133.0,132.5,131.0$, $128.6,128.5,128.0,127.8,114.1,94.0,63.9,55.2,45.9,37.5,34.9$.

HRMS (ESI) m/z: [M+Na] $]^{+}$Calcd for $\mathrm{C}_{25} \mathrm{H}_{23} \mathrm{INaO}_{4}$ 537.0539; Found: 537.0532 .

(d) Sonogashira coupling of $\mathbf{3 a}$

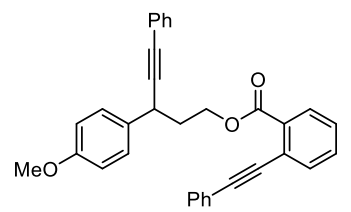

3-(4-Methoxyphenyl)-5-phenylpent-4-yn-1-yl 2-(phenylethynyl)benzoate (4c)

A suspension of iodobenzene 3a (49.6 mg, $0.1 \mathrm{mmol}), \mathrm{Pd}\left(\mathrm{PPh}_{3}\right)_{2} \mathrm{Cl}_{2}(3.5 \mathrm{mg}, 5 \mathrm{~mol} \%), \mathrm{CuI}(2.0$ $\mathrm{mg}, 10 \mathrm{~mol} \%), \mathrm{Et}_{3} \mathrm{~N}$ (40 $\mu \mathrm{L}, 2.0$ equiv) in $1.5 \mathrm{~mL}$ of DCE was degassed. After $5 \mathrm{~min}$, a solution of phenyl acetylene (12 mg, $1.1 \mathrm{eq})$ in DCE $(0.5 \mathrm{~mL})$ was added dropwise over $5 \mathrm{~min}$ via syringe and the reaction mixture was heated at $60{ }^{\circ} \mathrm{C}$ for $4 \mathrm{~h}$. After total consumption of the iodobenzene, the reaction mixture was filtered through celite and extracted with EtOAc $(3 \times 5 \mathrm{~mL})$. The organic layer was washed with a saturated solution of $\mathrm{NH}_{4} \mathrm{Cl}(2 \times 5 \mathrm{~mL})$, water $(2 \times 5 \mathrm{~mL})$, dried over $\mathrm{Na}_{2} \mathrm{SO}_{4}$ and the solvent was removed under vacuo. The reaction mixture was purified by flash 
chromatography on silica gel (pentane/EtOAc) to give the desired Sonogashira product $\mathbf{4 c}$ as a brown liquid (37.2 mg, 79\% yield)

${ }^{1} \mathrm{H} \mathrm{NMR}\left(300 \mathrm{MHz}, \mathrm{CDCl}_{3}\right): \delta 7.88(\mathrm{dt}, J=7.9,2.1 \mathrm{~Hz}, 1 \mathrm{H}), 7.57(\mathrm{dd}, J=7.7,1.5 \mathrm{~Hz}, 1 \mathrm{H}), 7.51-7.44$ (m, 2H), $7.41(\mathrm{td}, J=7.6,1.5 \mathrm{~Hz}, 2 \mathrm{H}), 7.34-7.18(\mathrm{~m}, 10 \mathrm{H}), 6.80-6.64(\mathrm{~m}, 2 \mathrm{H}), 4.59-4.32(\mathrm{~m}, 2 \mathrm{H})$, $3.98(\mathrm{t}, J=7.5 \mathrm{~Hz}, 1 \mathrm{H}), 3.69(\mathrm{~s}, 3 \mathrm{H}), 2.28-2.12(\mathrm{~m}, 2 \mathrm{H})$.

${ }^{13} \mathrm{C} \mathrm{NMR}\left(76 \mathrm{MHz}, \mathrm{CDCl}_{3}\right): \delta 166.2,158.6,134.2,133.1,131.8,131.7,130.6,128.6,128.5,128.4,128.3$, 128.2, 128.1, 127.9, 127.9, 123.7, 123.5, 123.3, 114.1, 94.4, 90.6, 88.4, 83.8, 63.2, 55.3, 37.3, 34.4.

HRMS (ESI) m/z: [M+Na] $]^{+}$Calcd for $\mathrm{C}_{33} \mathrm{H}_{26} \mathrm{NaO}_{3}$ 493.1780; Found: 493.1770.

(e) Cyclization of 3u

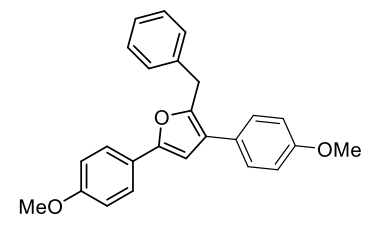

\section{2,3-Bis(4-methoxyphenyl)-5-phenylfuran (4d)}

A solution of $3 \mathbf{u}(88.2 \mathrm{mg}, 0.15 \mathrm{mmol})$ in THF $(1.0 \mathrm{~mL})$ was added $\mathrm{NaOH}$ aq $(1 \mathrm{~mL}, 1 \mathrm{M})$ solution. The reaction mixture was stirred at $80{ }^{\circ} \mathrm{C}$ for $6 \mathrm{~h}$. After completion, the mixture was extracted with ethyl acetate $(3 \times 3 \mathrm{~mL})$. The organic layer was dried by $\mathrm{Na}_{2} \mathrm{SO}_{4}$ and evaporated under reduced pressure to afford the alcohol derivative S1 (directly used for next step).

A solution of $\mathbf{S 1}$ in $\mathrm{MeCN}(1.0 \mathrm{~mL})$ was added IBX. The reaction mixture was stirred at $80{ }^{\circ} \mathrm{C}$ for $12 \mathrm{~h}$. After completion, the mixture was filtered and evaporated under reduced pressure to afford the ketone derivative $\mathbf{S 2}$ (directly used for next step).

To an oven-dried flask under $\mathrm{N}_{2}$ was added compound 5a (1,3,5-triphenylpent-4-yn-1-one, $311 \mathrm{mg}$, $1.0 \mathrm{mmol})$. The substrate was dissolved in toluene $(2.5 \mathrm{~mL})$, and TMSOTf $(180 \mu \mathrm{L}, 221 \mathrm{mg}, 1.0$ mmol) was added dropwise. The flask was fitted with a reflux condenser and heated at reflux $\left(110^{\circ} \mathrm{C}\right)$ for $2 \mathrm{~h}$. The reaction mixture was allowed to cool to room temperature, then diluted with $\mathrm{Et}_{2} \mathrm{O}$ (150 $\mathrm{mL})$, and washed with saturated $\mathrm{NaHCO}_{3}(50 \mathrm{~mL})$. The layers were separated, and the organic layer was dried over $\mathrm{MgSO}_{4}$. The solution was filtered, and the solvent was removed in vacuo. The title compound was purified by column chromatography on silica gel (0-2\% EtOAc/hexanes) to yield the $4 \mathbf{d}$ as yellow oil ( $30.5 \mathrm{mg}, 55 \%$ yield).

${ }^{1} \mathrm{H} \mathrm{NMR}\left(300 \mathrm{MHz}, \mathrm{CDCl}_{3}\right): \delta 7.73-7.49(\mathrm{~m}, 2 \mathrm{H}), 7.39-7.27(\mathrm{~m}, 6 \mathrm{H}), 6.94(\mathrm{td}, J=6.5,5.9,1.8 \mathrm{~Hz}$, 4H), $6.67(\mathrm{~s}, 1 \mathrm{H}), 4.19(\mathrm{~s}, 2 \mathrm{H}), 3.85(\mathrm{~d}, J=1.4 \mathrm{~Hz}, 6 \mathrm{H})$. 
${ }^{13} \mathrm{C}$ NMR $\left(126 \mathrm{MHz}, \mathrm{CDCl}_{3}\right): \delta 158.9,158.3,152.3,147.7,138.8,128.8,128.6,128.3,126.4,126.3$, $125.0,123.9,123.9,114.1,105.2,55.3,55.2,32.9$.

This compound was known and spectroscopic data is in accordance with the described in literature. ${ }^{[7]}$

\section{E. Mechanistic studies:}

(a) Radical trapping experiment:

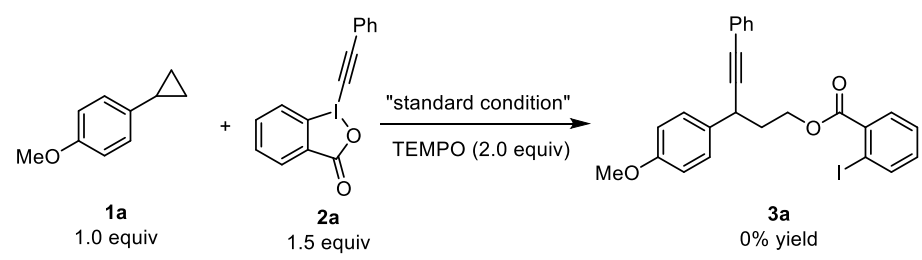

To an oven-dried $10 \mathrm{~mL}$ Schlenk tube equipped with a stir bar, aryl cyclopropanes $\mathbf{1 a}(29.6 \mathrm{mg}, 0.20$ mmol, 1.0 equiv), EBX 2a (104.4 mg, $0.30 \mathrm{mmol}, 1.5$ equiv), photocatalyst 4CzIPN (15.8 mg, 10.0 mol\%), BI-OAc (30.6 mg, $50.0 \mathrm{~mol} \%)$ and (2,2,6,6-Tetramethylpiperidin-1-yl)oxyl (TEMPO) (62.5 mg, 2.0 equiv). Then the reaction tube was evacuated and backfilled with argon for two times. Subsequently, anhydrous $\mathrm{CHCl}_{3}(2.0 \mathrm{~mL}, 0.1 \mathrm{M})$ were added. The resulting mixture was irradiated under blue LED at room temperature for $24 \mathrm{~h}$. The mixture was detected by crude NMR.

(b) Reaction component analysis experiment:
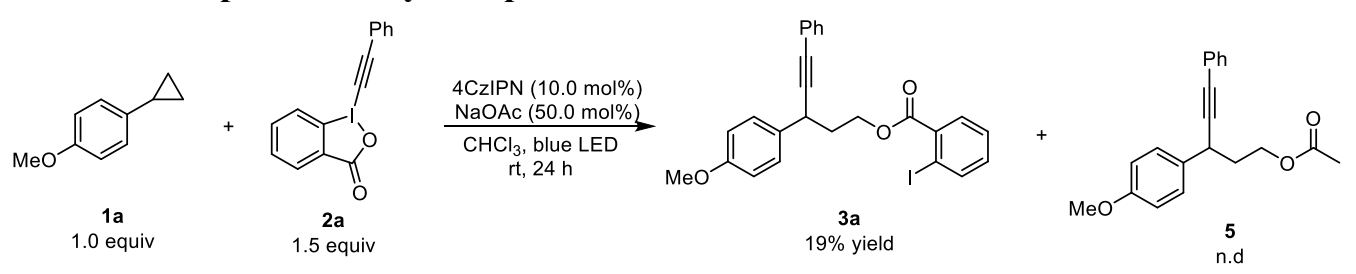

To an oven-dried $10 \mathrm{~mL}$ Schlenk tube equipped with a stir bar, aryl cyclopropanes $\mathbf{1 a}(14.8 \mathrm{mg}, 0.10$ mmol, 1.0 equiv), EBX 2a (52.2 mg, 0.15 mmol, 1.5 equiv), photocatalyst 4CzIPN (7.9 mg, $10.0 \mathrm{~mol} \%)$ and $\mathrm{NaOAc}$ ( $4.1 \mathrm{mg}, 0.10 \mathrm{mmol}, 0.5$ equiv). Then the reaction tube was evacuated and backfilled with argon for two times. Subsequently, anhydrous $\mathrm{CHCl}_{3}(1.0 \mathrm{~mL}, 0.1 \mathrm{M})$ were added. The resulting mixture was irradiated under blue LED at room temperature for $24 \mathrm{~h}$. The residue was checked by crude NMR and then purified by silica gel chromatography to afford the desired product $\mathbf{3 a}$ in $19 \%$ yield.

\section{(c) Luminescence quenching experiments}

Emission intensities were recorded using a Jasco FP-8300 spectrofluorometer. 4-CzIPN solutions were excited at $435 \mathrm{~nm}$ and the emission intensity was recorded at $590 \mathrm{~nm}$. In a typical experiment, to a certain amount of a solution of 4-CzIPN in $\mathrm{CHCl}_{3}(3 \mathrm{~mL})$, the appropriate amount of quencher (1-cyclopropyl4-methoxybenzene 1a and BIOAc) was added in a screw-top quartz cuvette, and the emission of the 
sample was recorded.

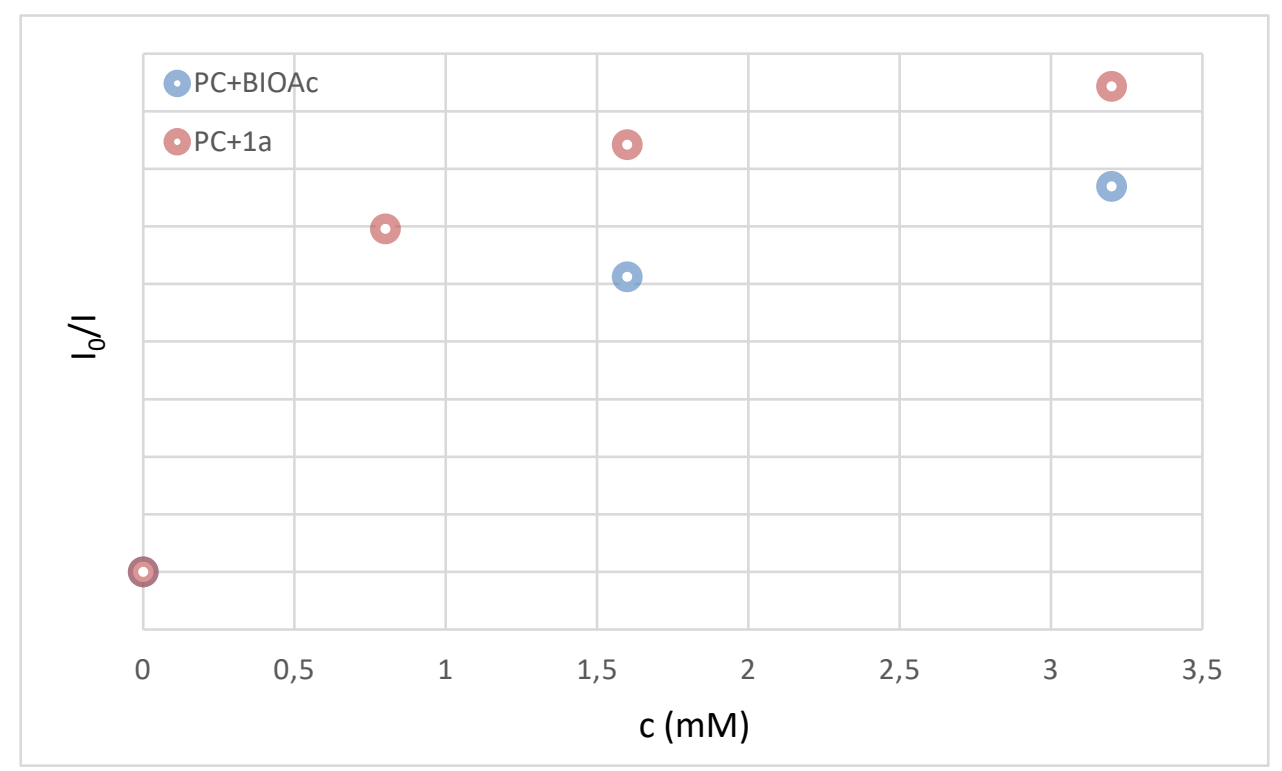

\section{G. References:}

[1] D. J. Wallace, C. Chen, Tetrahedron Lett. 2002, 43, 6987.

[2] S. Francoeur, J. Martel, N. Wilb, A. B. Charette, Angew. Chem. Int. Ed. 2020, 59, 4539.

[3] M. Sugawara, J. I. Yoshida, J. Am. Chem. Soc. 1997, 119, 11986.

[4] F. Le. Vaillant, M. Garreau, S. Nicolai, G. Gryn'ova, C. Corminboeuf, J. Waser, Chem. Sci., 2018, 9, 5883.

[5] H. China, N. Kageyama, H. Yatabe, N. Takenaga, T. Dohi, Molecules 2021, 26, 1897.

[6] S. G. E. Amos, S. Nicolai, J. Waser, Chem. Sci., 2020, 11, 11274.

[7] T. Wang, X. Chen, L. Chen, Z. Zhan, Org. Lett. 2011, 13, 3324 


\section{H. NMR spectrum:}

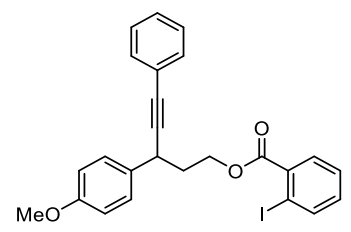

(3a)

\subsection{5}
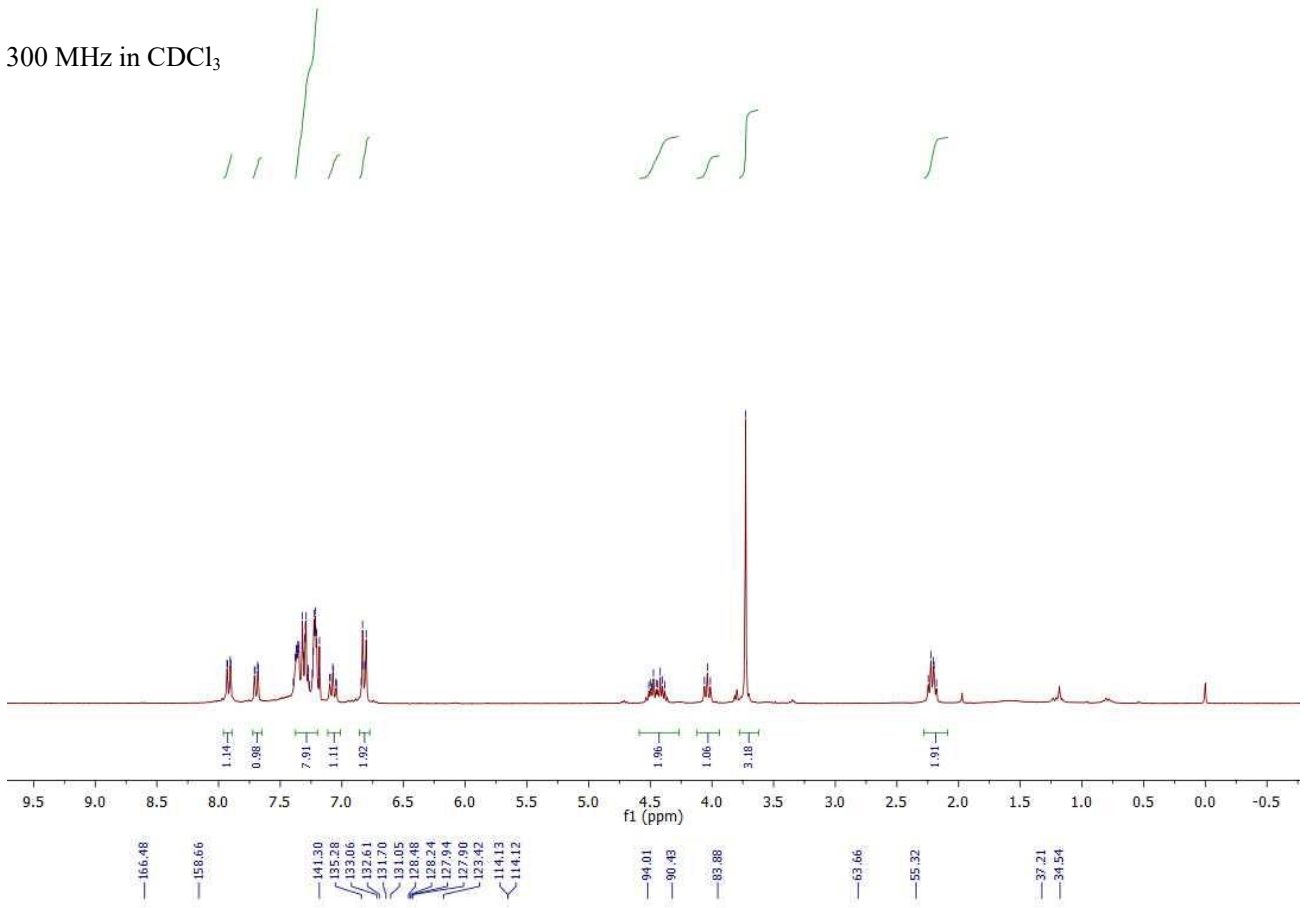

$126 \mathrm{MHz}$ in $\mathrm{CDCl}_{3}$

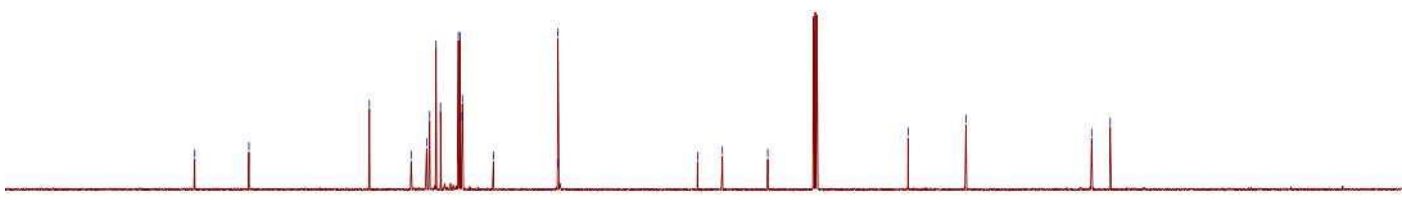

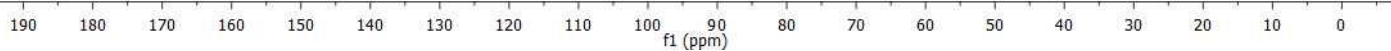




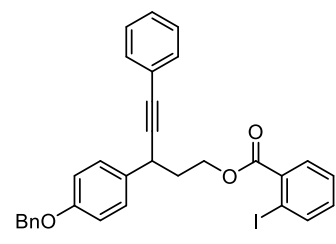

(3b)

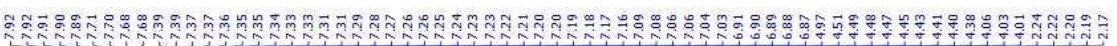
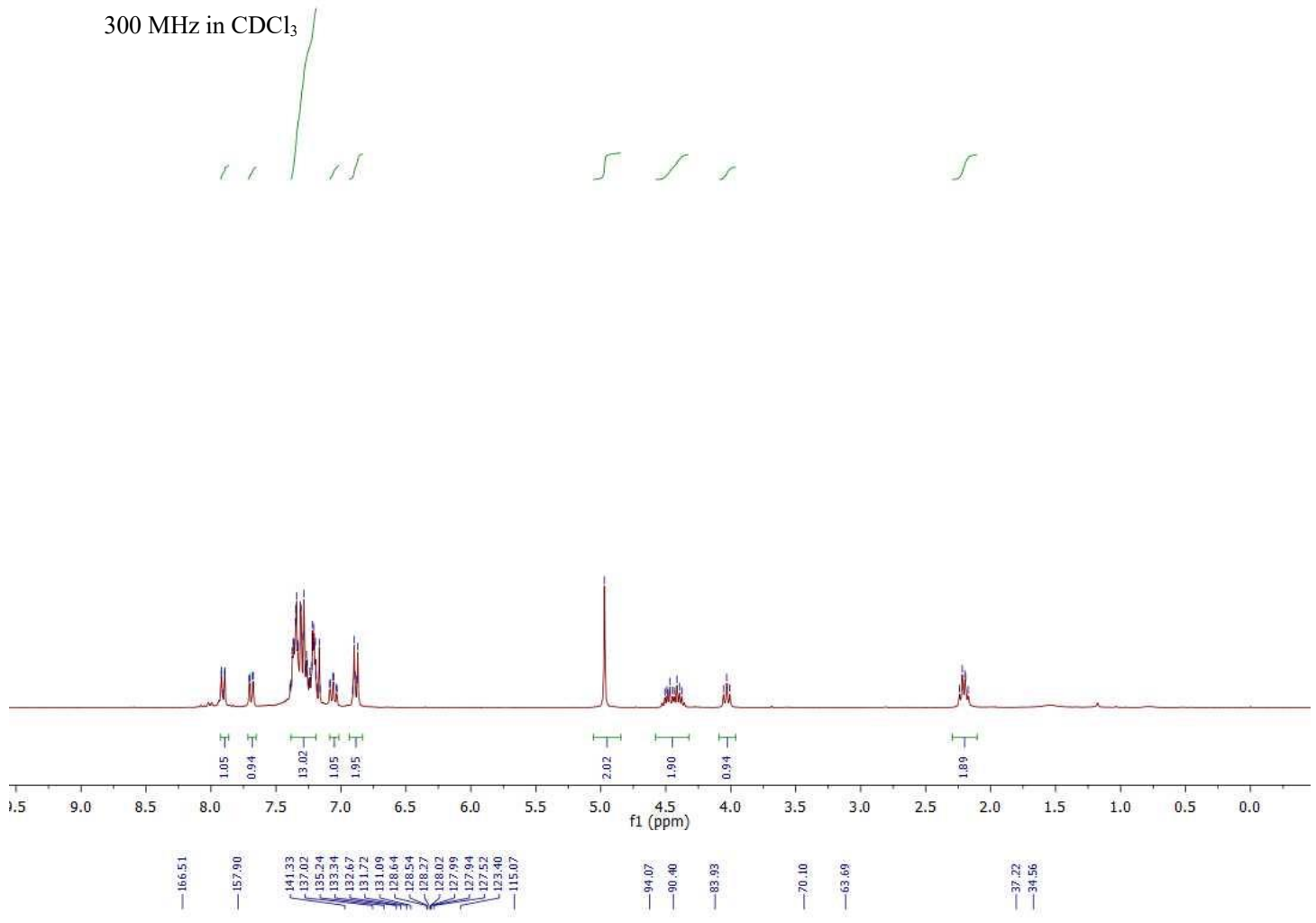

$76 \mathrm{MHz}$ in $\mathrm{CDCl}_{3}$

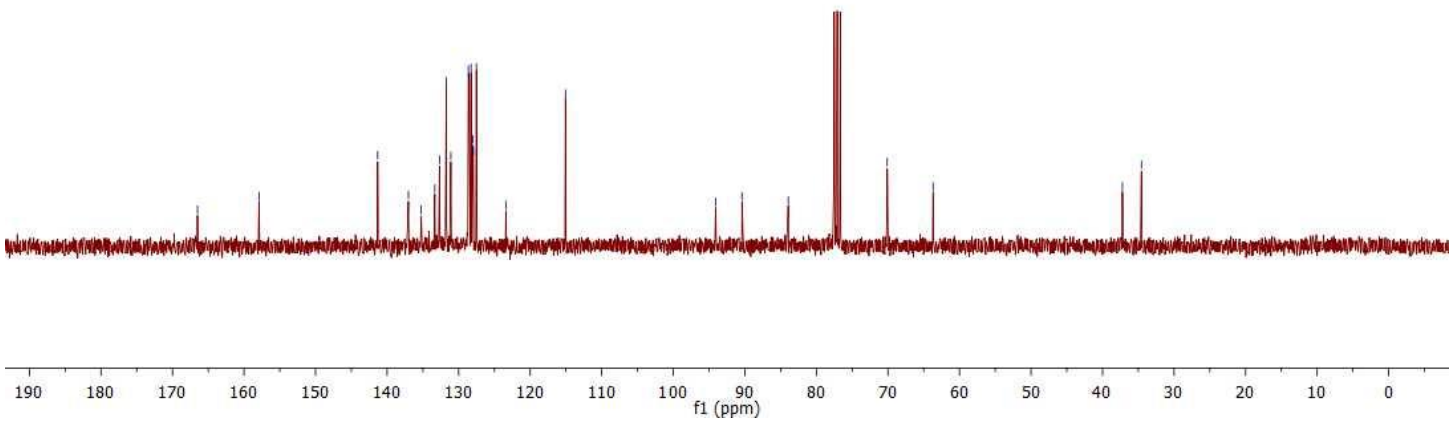

S31 


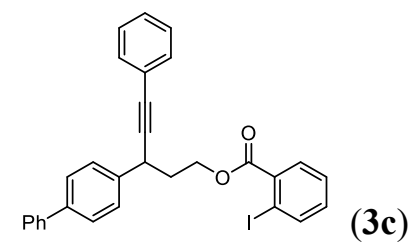

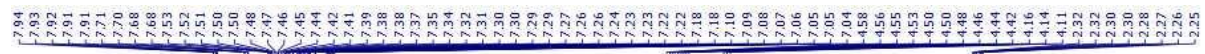
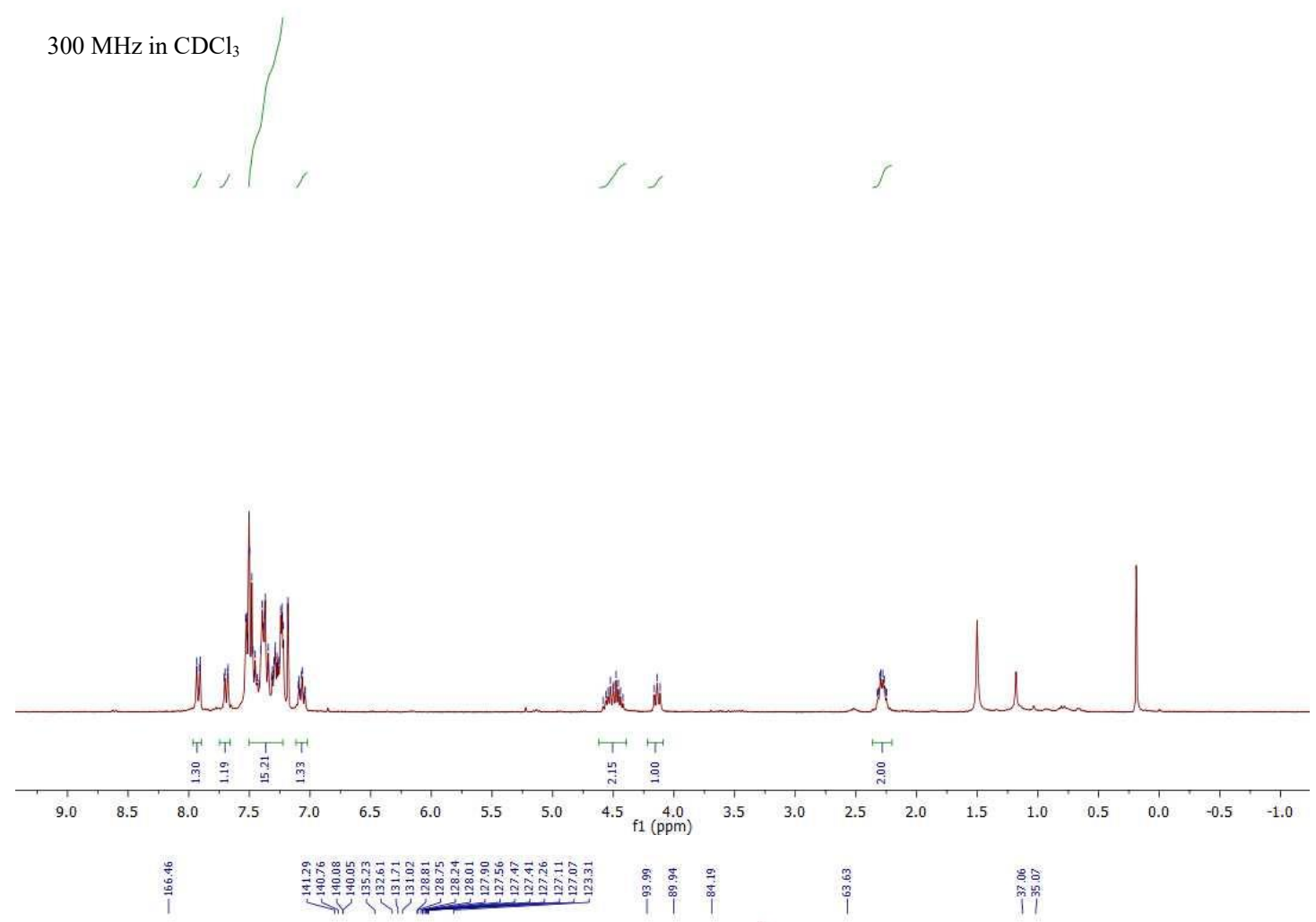

$151 \mathrm{MHz}$ in $\mathrm{CDCl}_{3}$

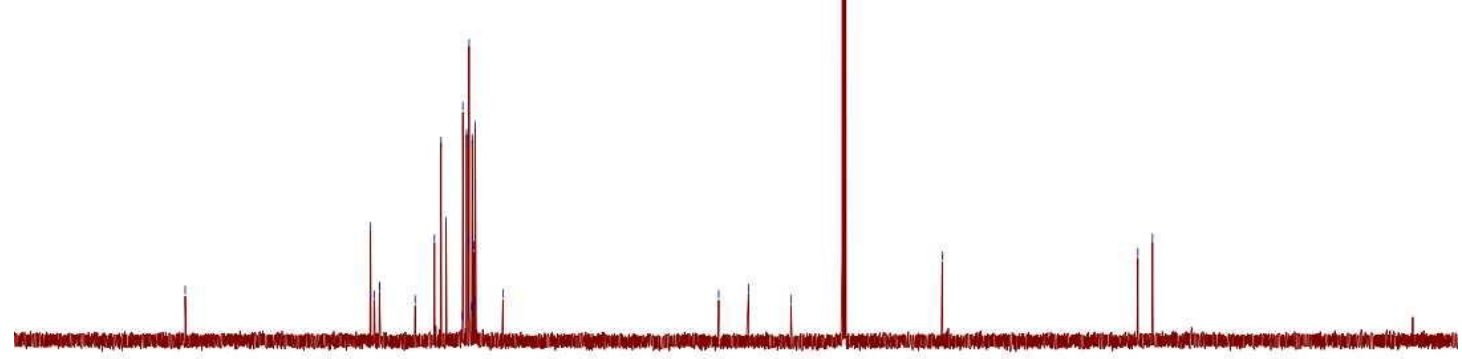

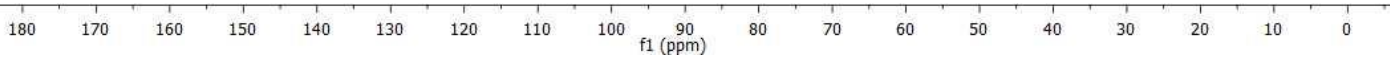


<smiles>Cc1ccc(C(=Cc2ccccc2)CCOC(=O)c2ccccc2I)cc1</smiles>

(3d)

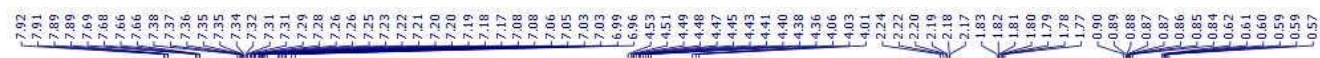

$300 \mathrm{MHz}$ in $\mathrm{CDCl}_{3}$
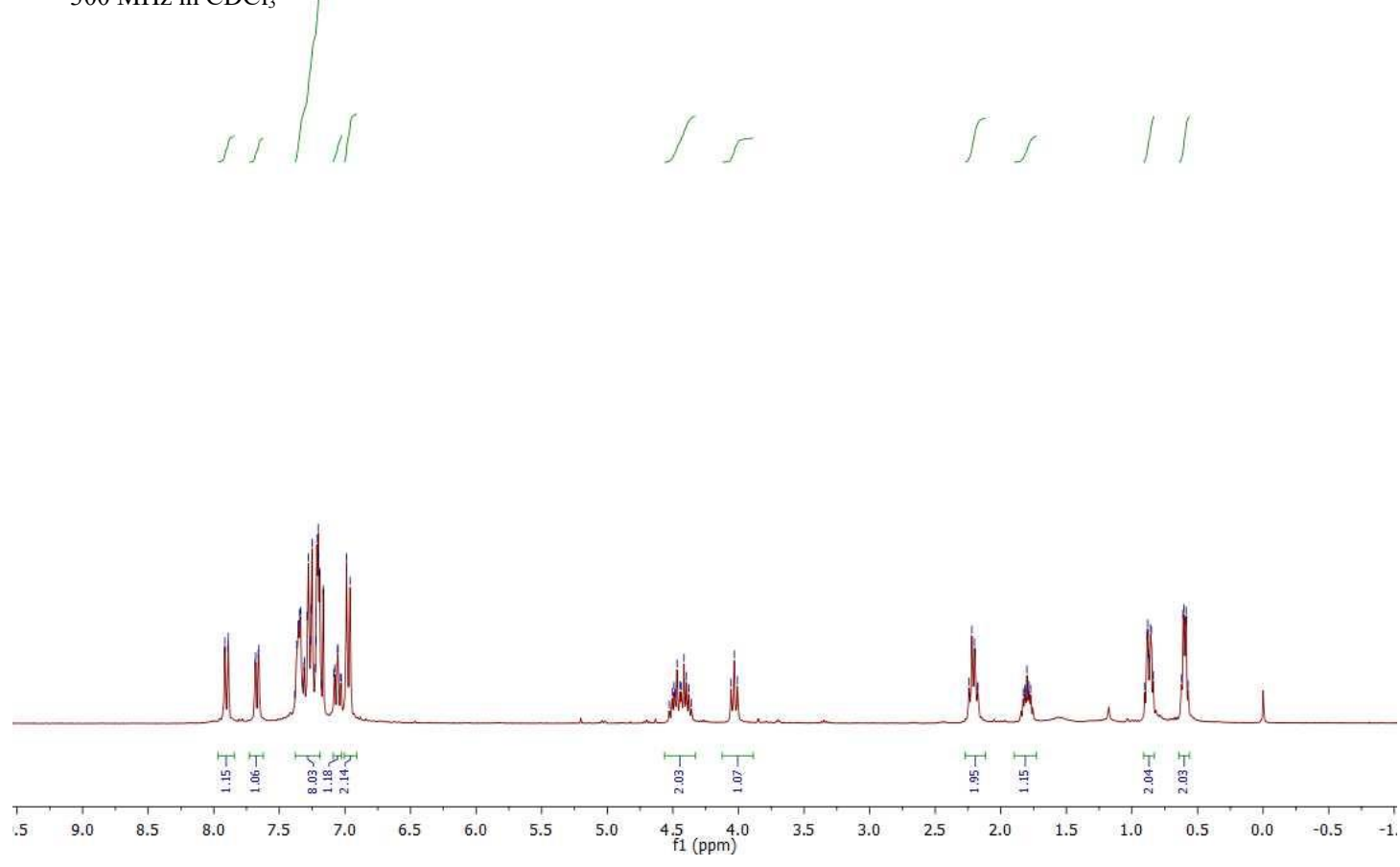

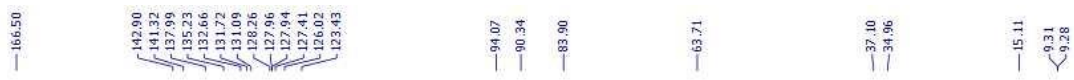

$76 \mathrm{MHz}$ in $\mathrm{CDCl}_{3}$

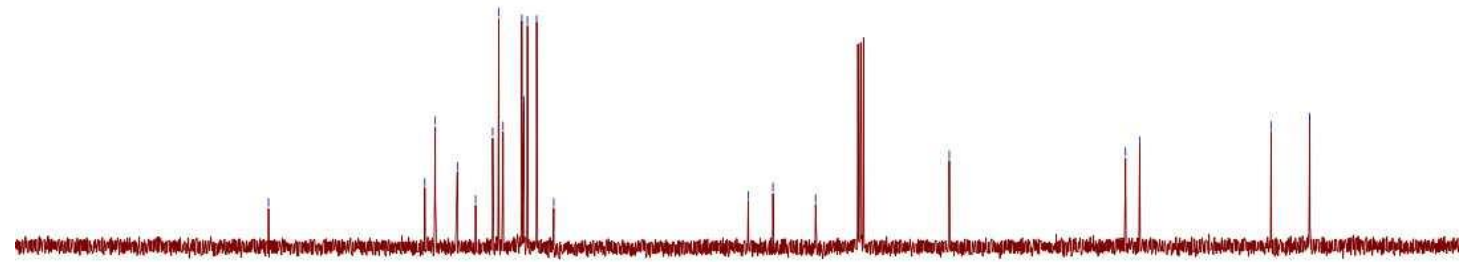

$\begin{array}{lllllllllll}200 & 190 & 180 & 170 & 160 & 150 & 140 & 130 & 120 & 110 & \begin{array}{l}100 \\ \mathrm{f} 1(\mathrm{ppm})\end{array}\end{array}$

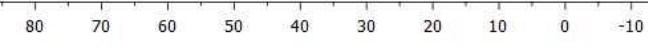




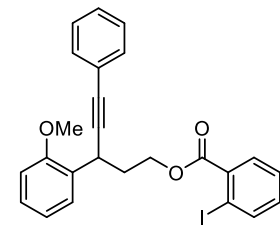

(3e)

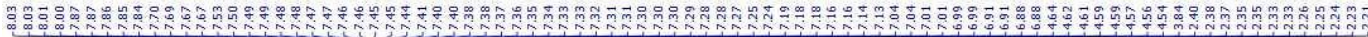

$300 \mathrm{MHz}$ in $\mathrm{CDCl}_{3}$
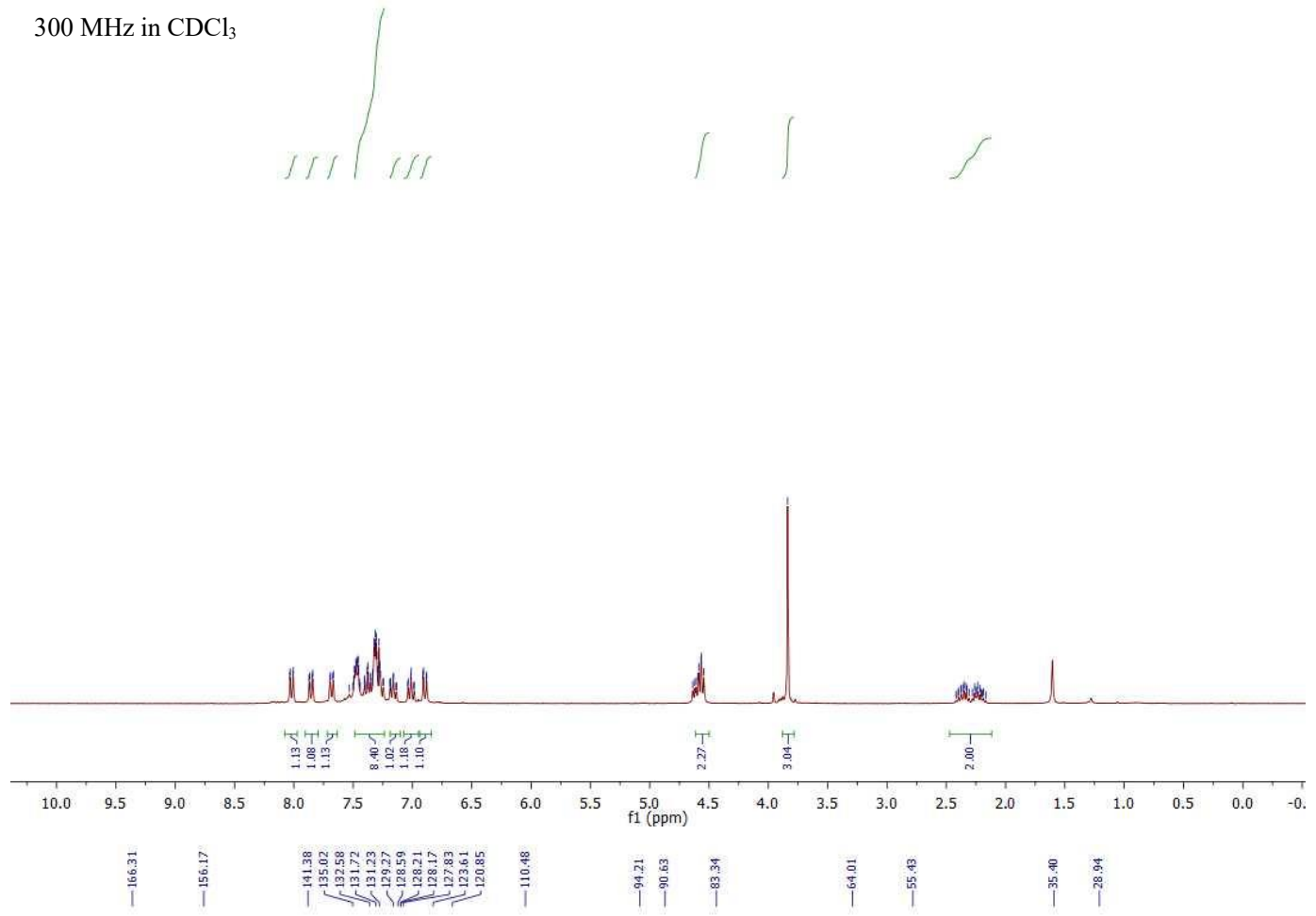

$126 \mathrm{MHz}$ in $\mathrm{CDCl}_{3}$

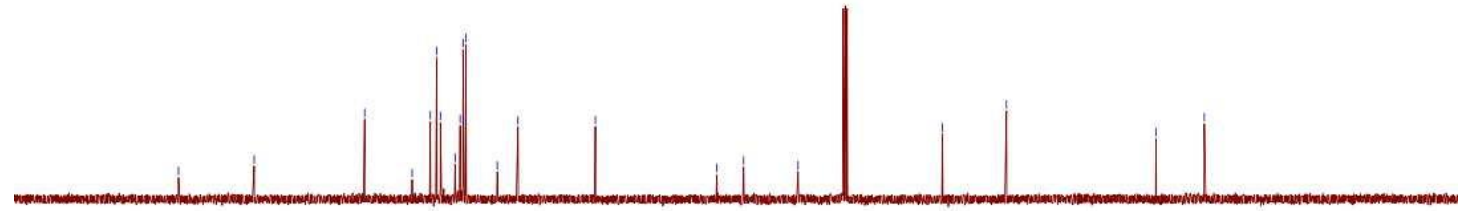

$\begin{array}{lllllllll}180 & 170 & 160 & 150 & 140 & 130 & 120 & 110 & 100 \\ \mathrm{f1} 1(\mathrm{ppm})\end{array}$ 


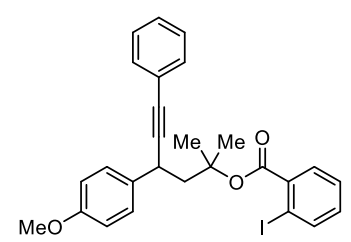

(3f)

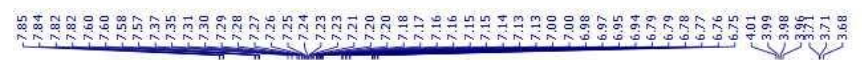

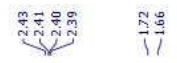

$300 \mathrm{MHz}$ in $\mathrm{CDCl}_{3}$
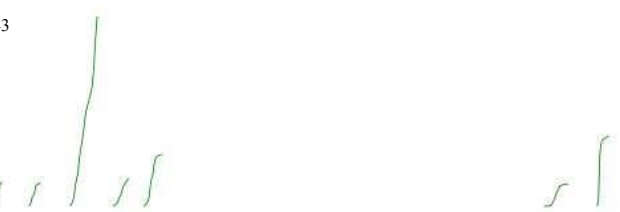

$\iiint$

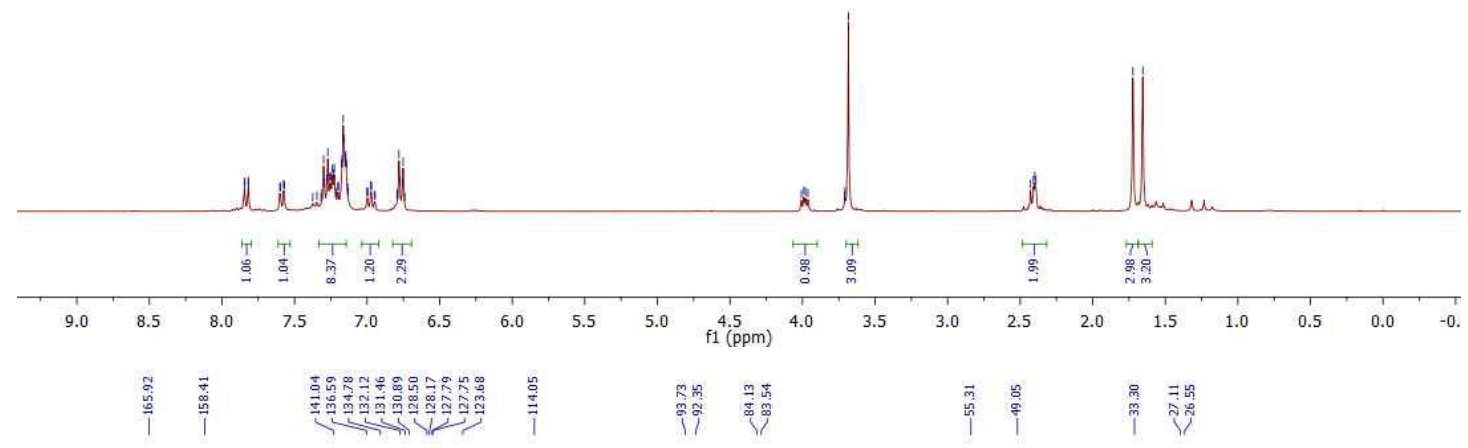

$76 \mathrm{MHz}$ in $\mathrm{CDCl}_{3}$

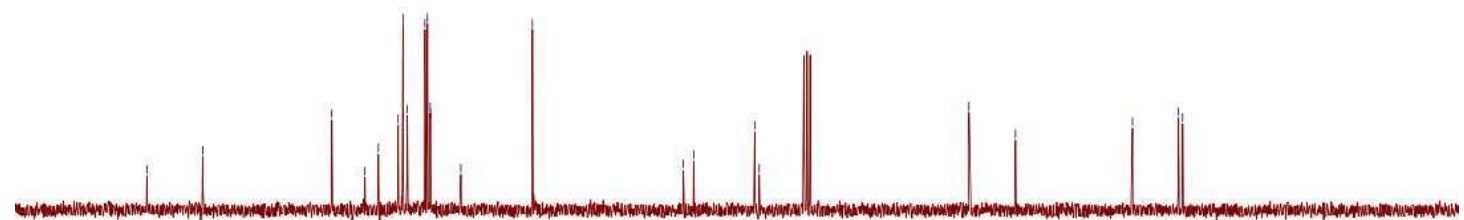

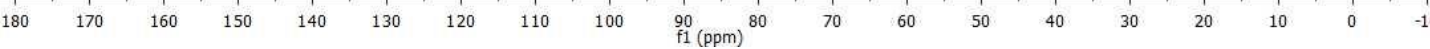




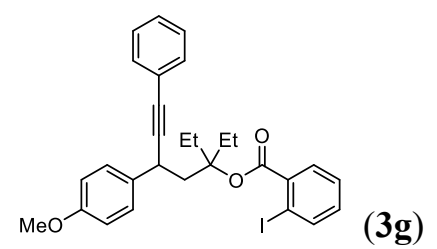

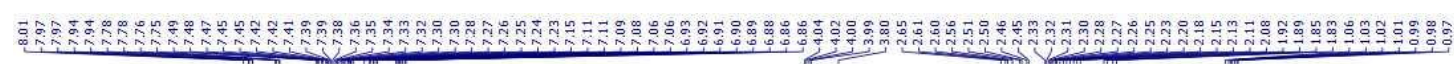

$300 \mathrm{MHz}$ in $\mathrm{CDCl}_{3}$

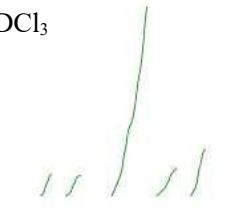

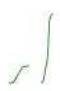
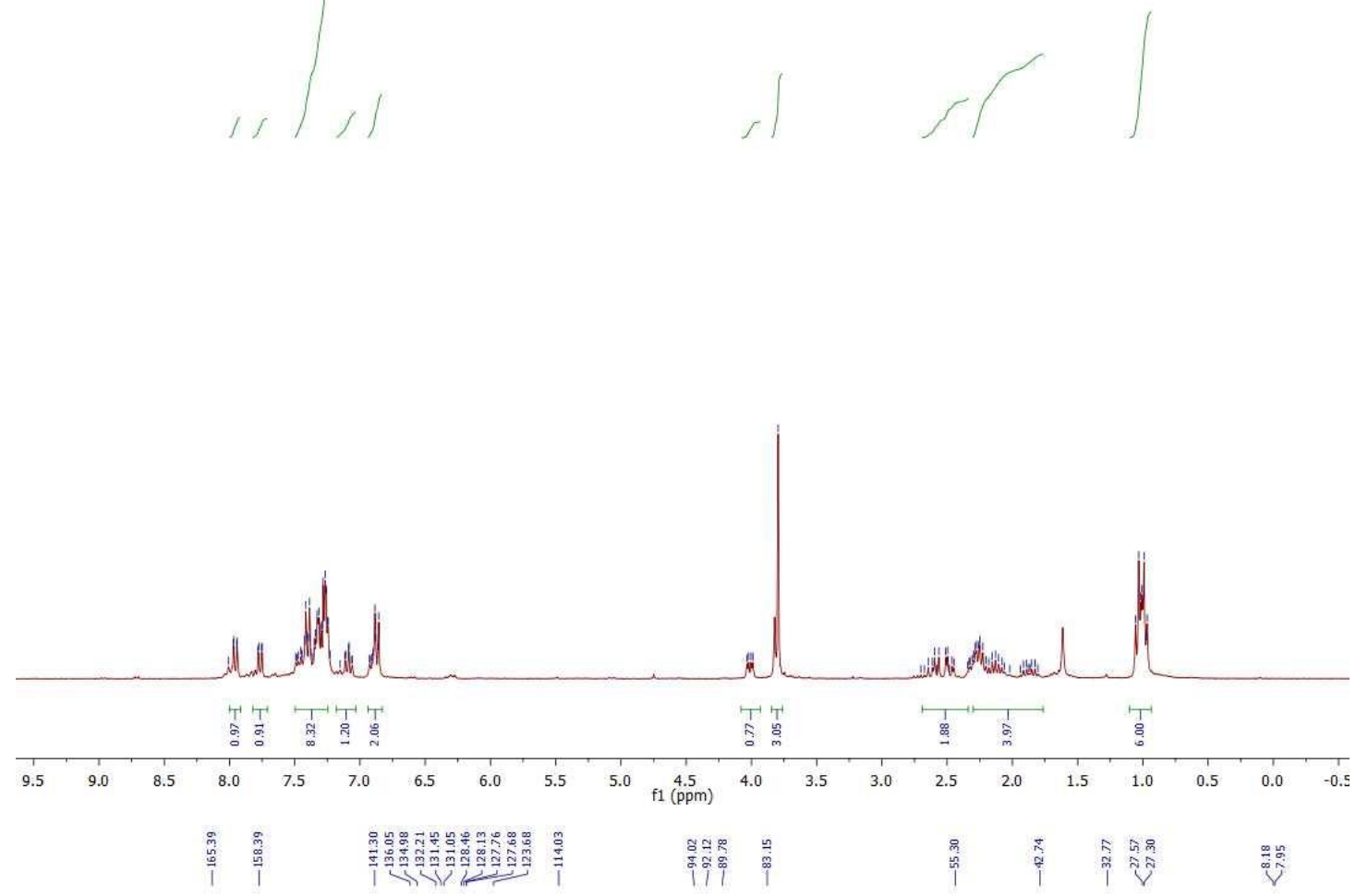

$76 \mathrm{MHz}$ in $\mathrm{CDCl}_{3}$

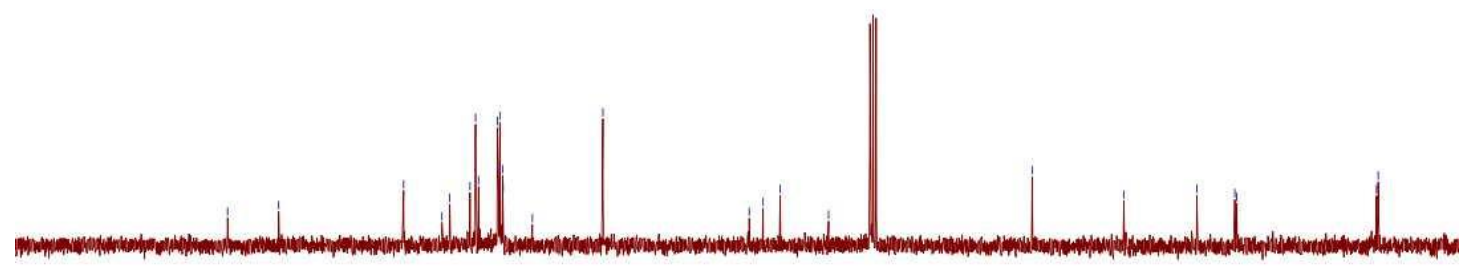

$\begin{array}{llllllllll}190 & 180 & 170 & 160 & 150 & 140 & 130 & 120 & 110 & \begin{array}{c}100 \\ \mathrm{f} 1(\mathrm{ppm})\end{array}{ }^{90}\end{array}$

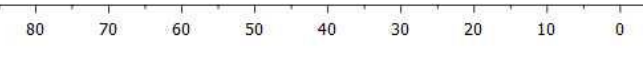




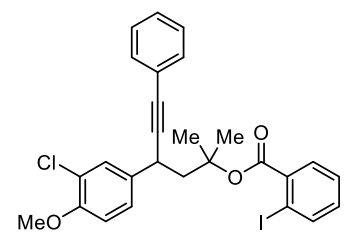

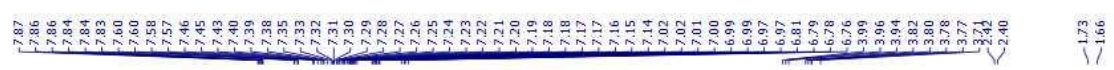

$300 \mathrm{MHz}$ in $\mathrm{CDCl}_{3}$

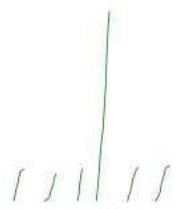

1
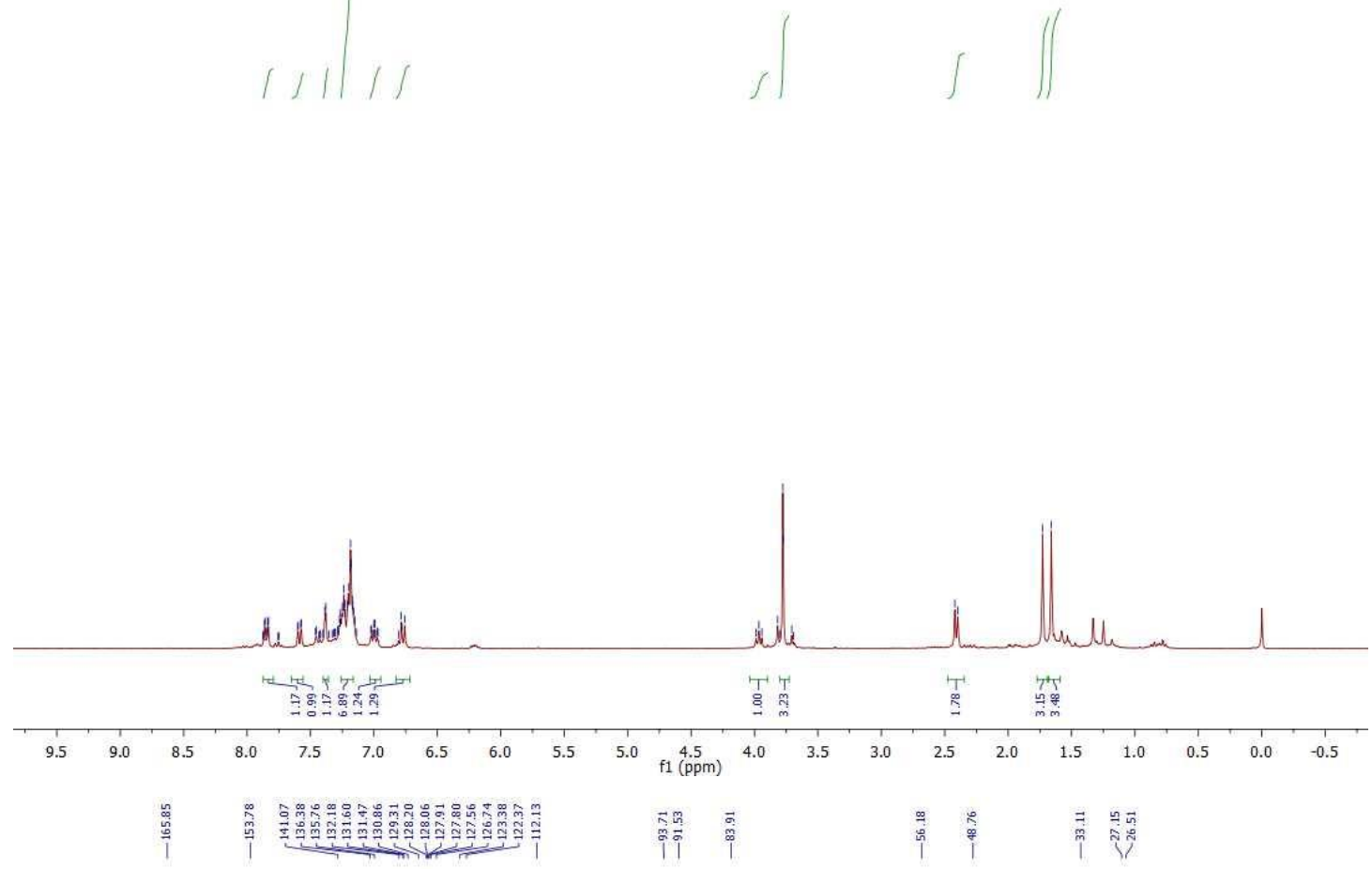

$76 \mathrm{MHz}$ in $\mathrm{CDCl}_{3}$

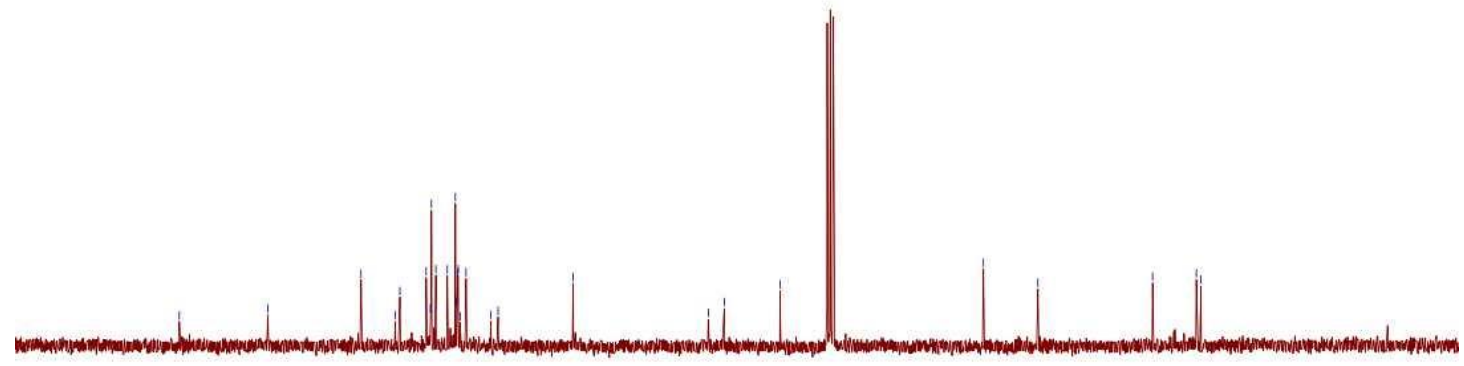

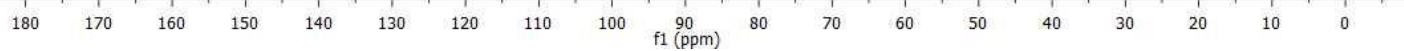




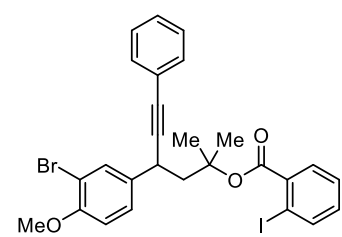

(3i)
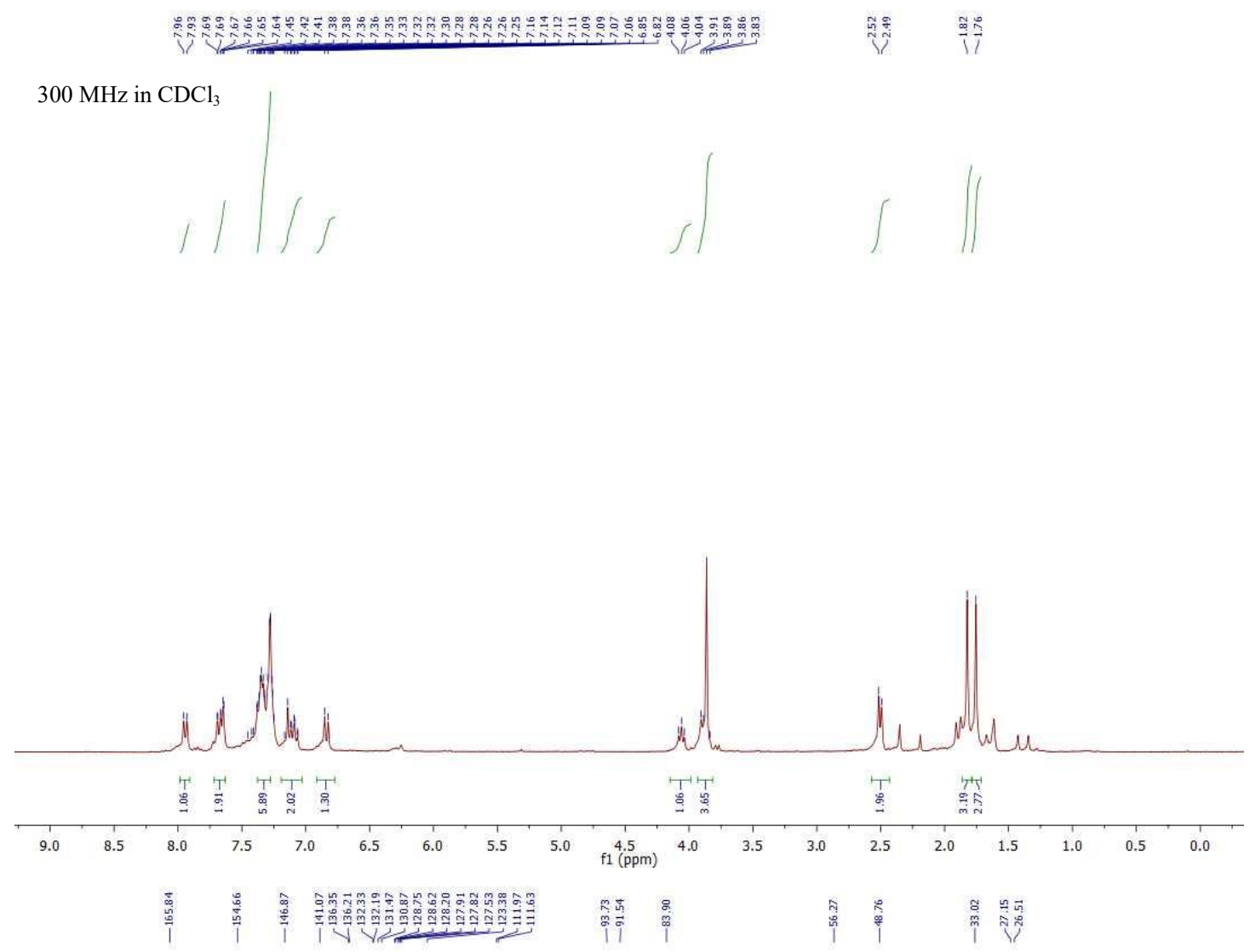

$76 \mathrm{MHz}$ in $\mathrm{CDCl}_{3}$

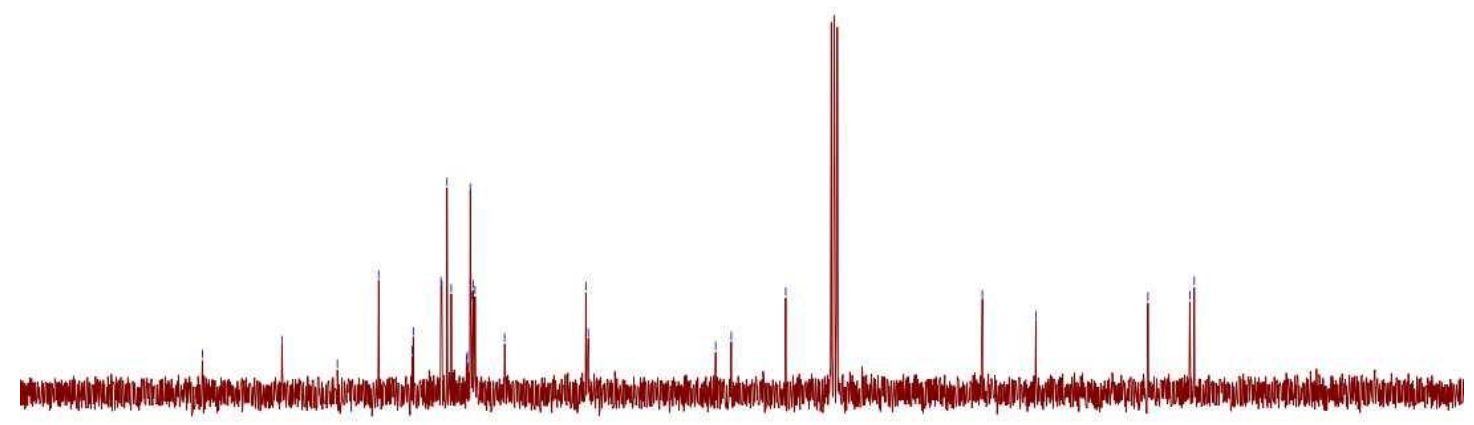

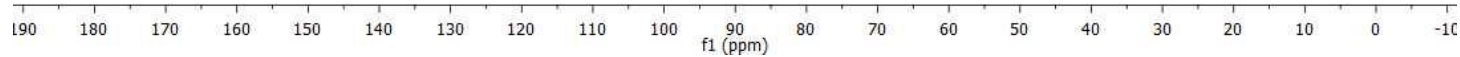




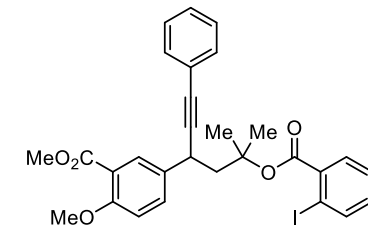

(3j)

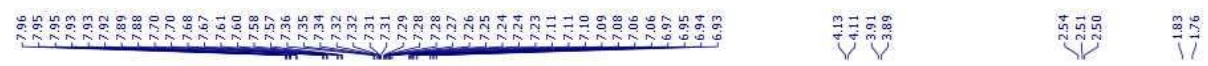

$300 \mathrm{MHz}$ in $\mathrm{CDCl}_{3}$

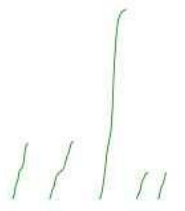

「

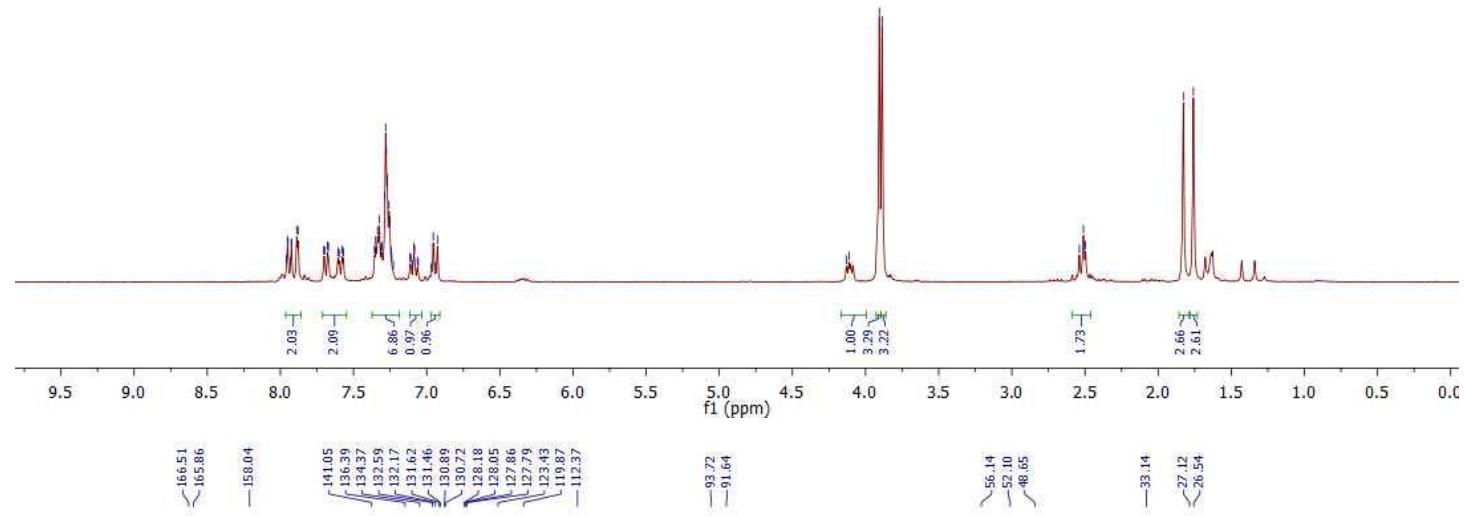

$76 \mathrm{MHz}$ in $\mathrm{CDCl}_{3}$

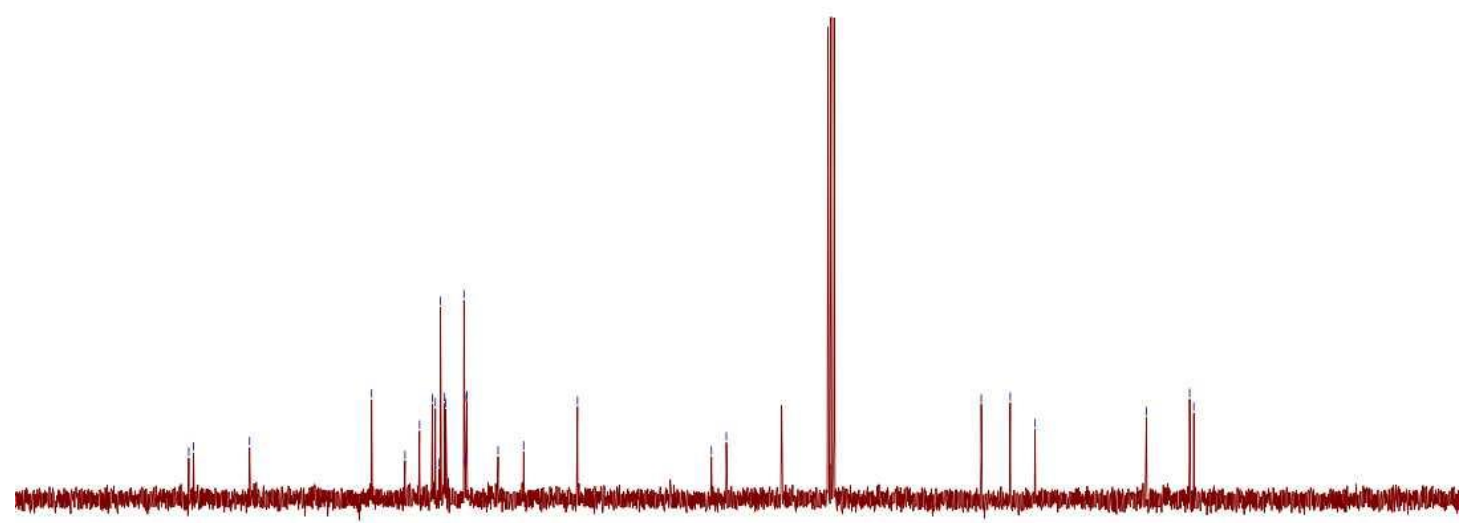

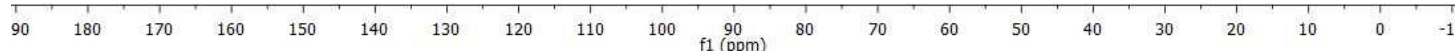




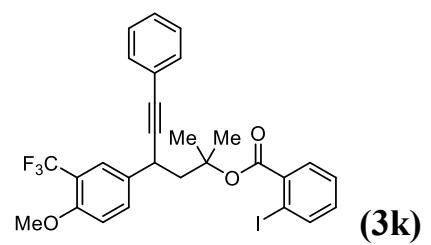

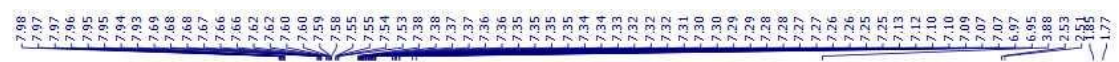

$300 \mathrm{MHz}$ in $\mathrm{CDCl}_{3}$
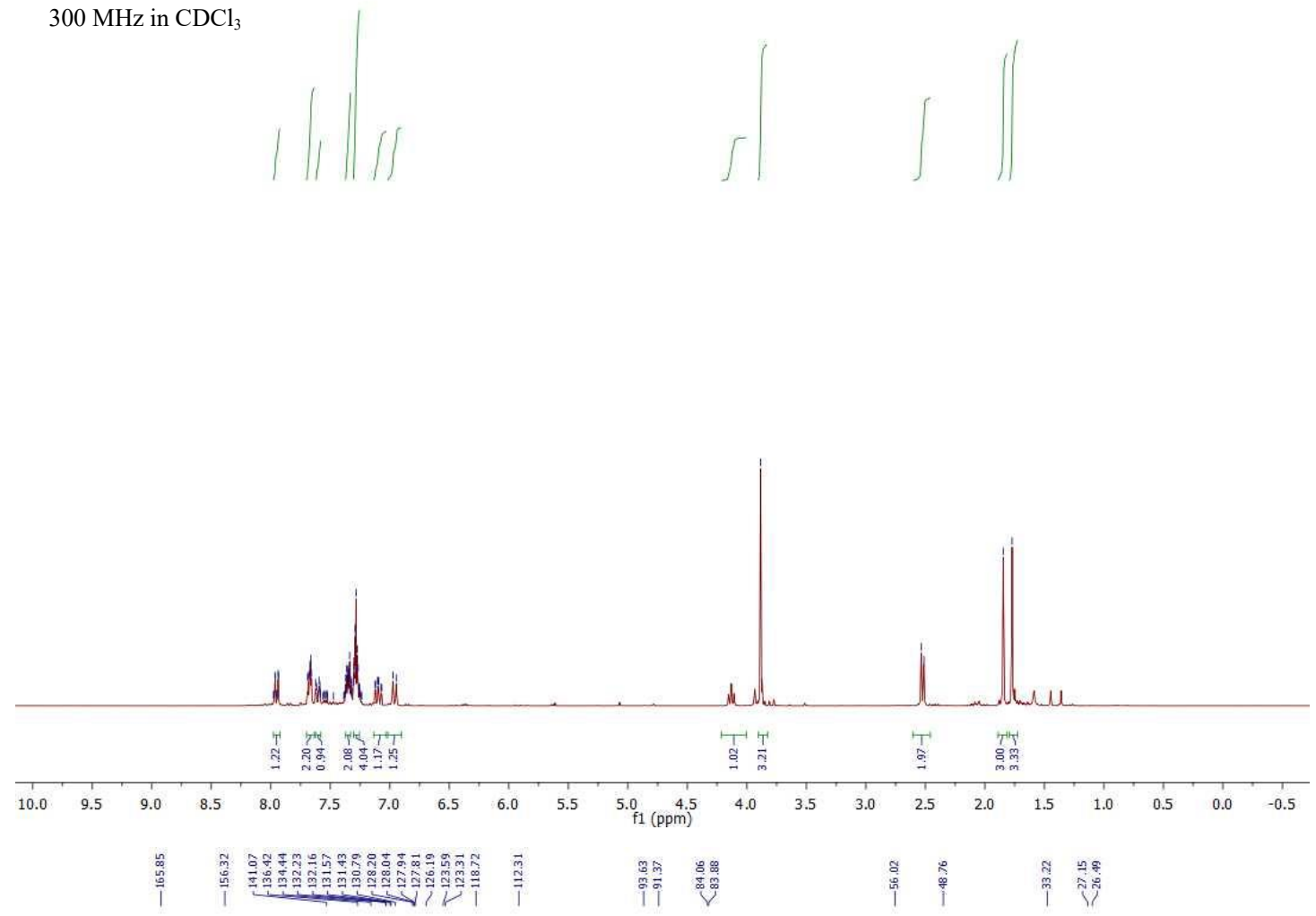

$126 \mathrm{MHz}$ in $\mathrm{CDCl}_{3}$

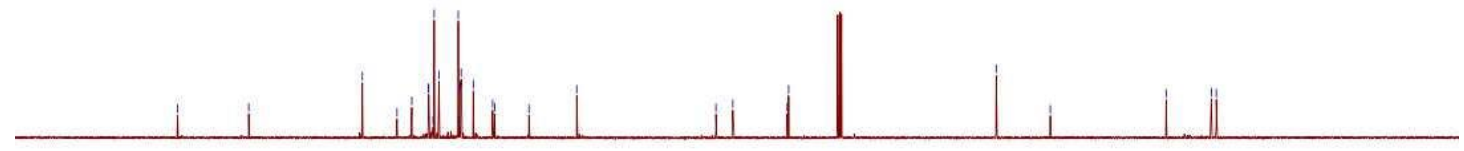

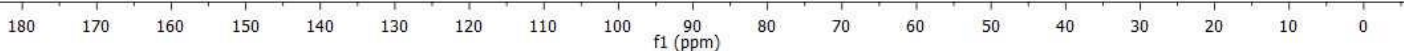


$282 \mathrm{MHz}$ in $\mathrm{CDCl}_{3}$

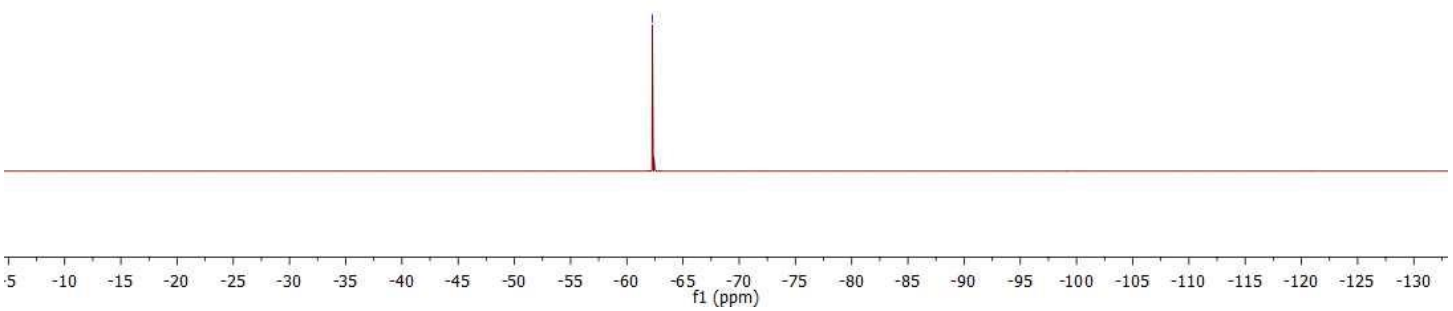




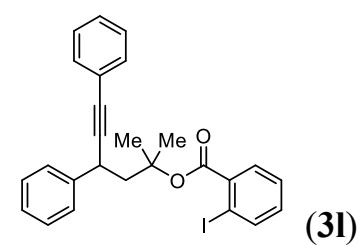

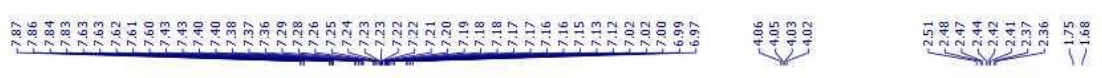

$300 \mathrm{MHz}$ in $\mathrm{CDCl}_{3}$
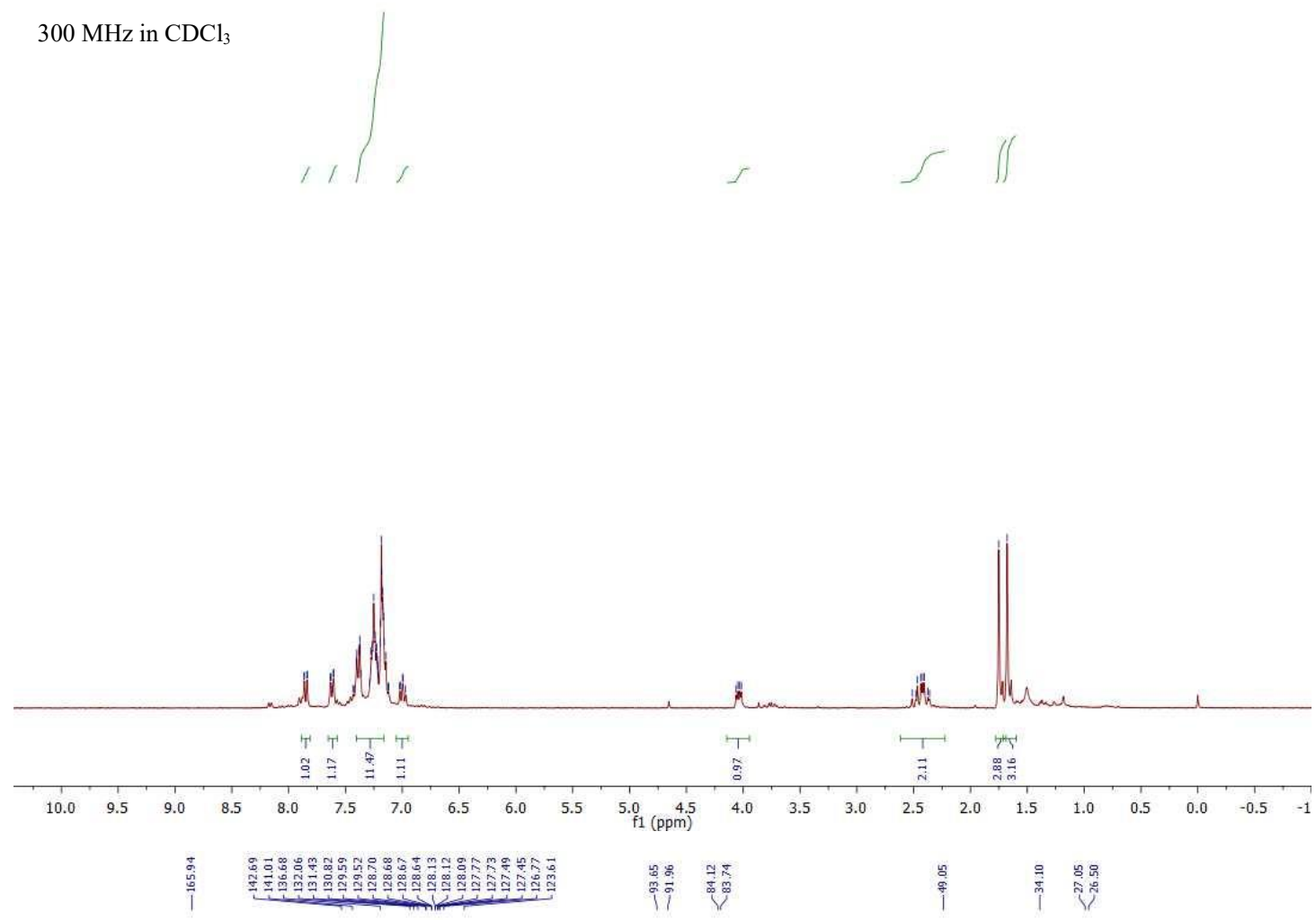

$151 \mathrm{MHz}$ in $\mathrm{CDCl}_{3}$

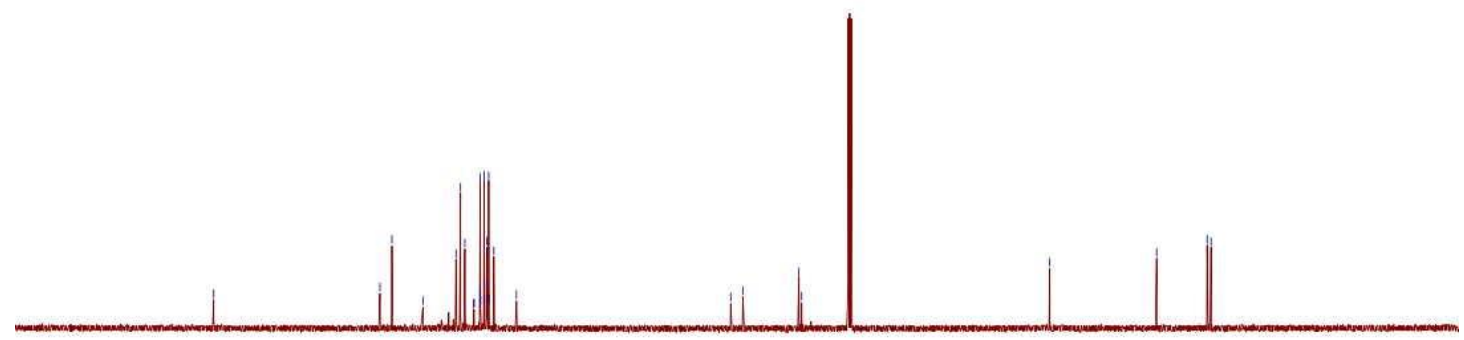

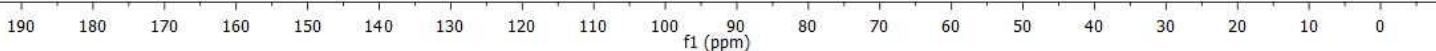




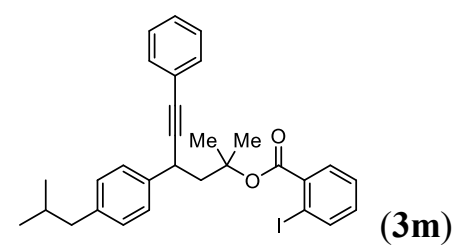

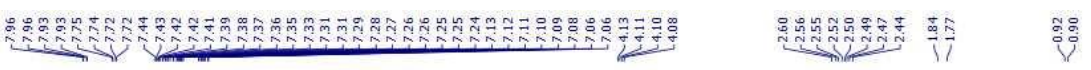

$300 \mathrm{MHz}$ in $\mathrm{CDCl}_{3}$
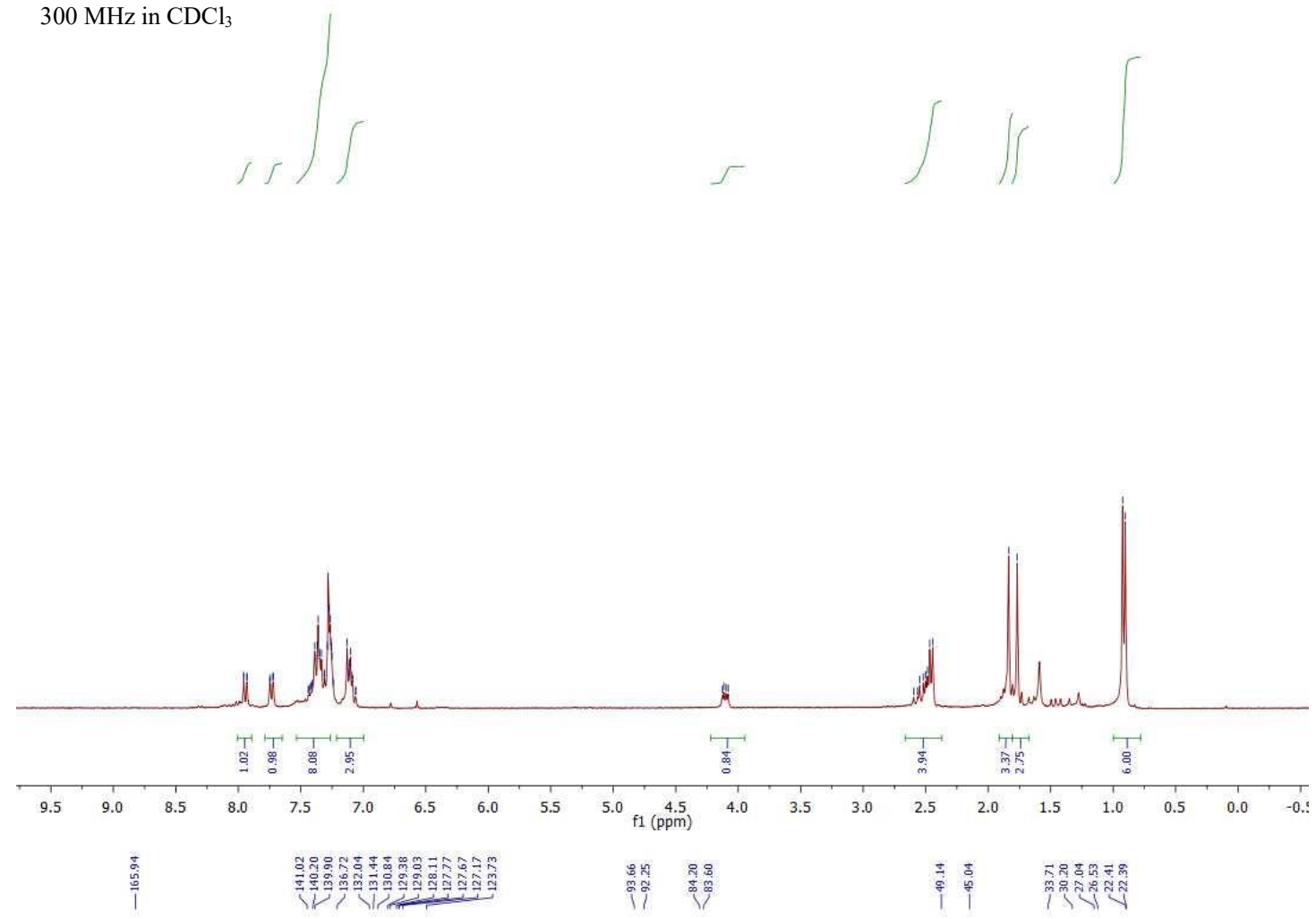

$126 \mathrm{MHz}$ in $\mathrm{CDCl}_{3}$
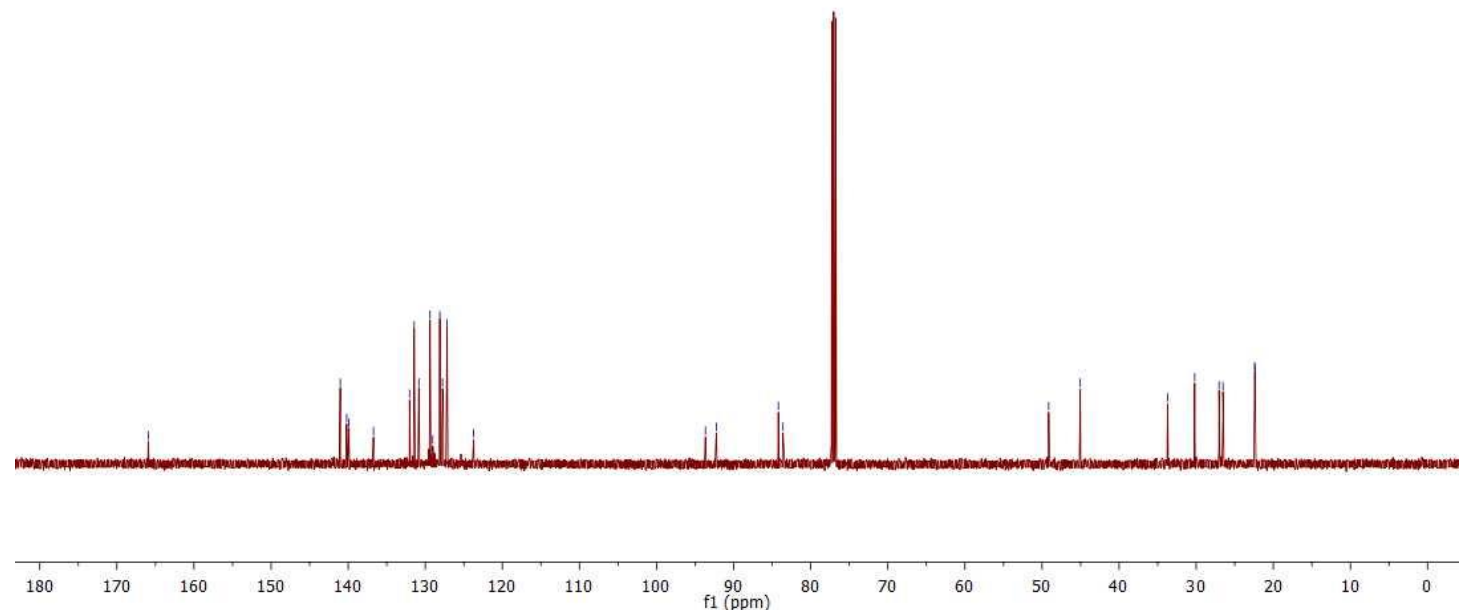


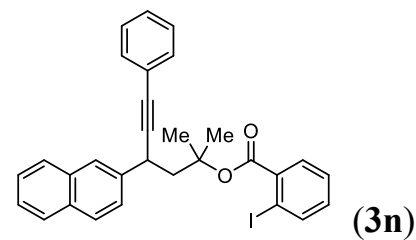

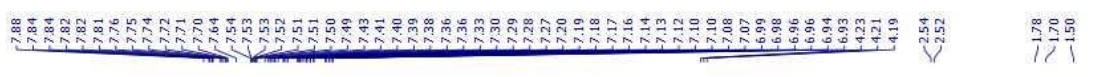

$300 \mathrm{MHz}$ in $\mathrm{CDCl}_{3}$
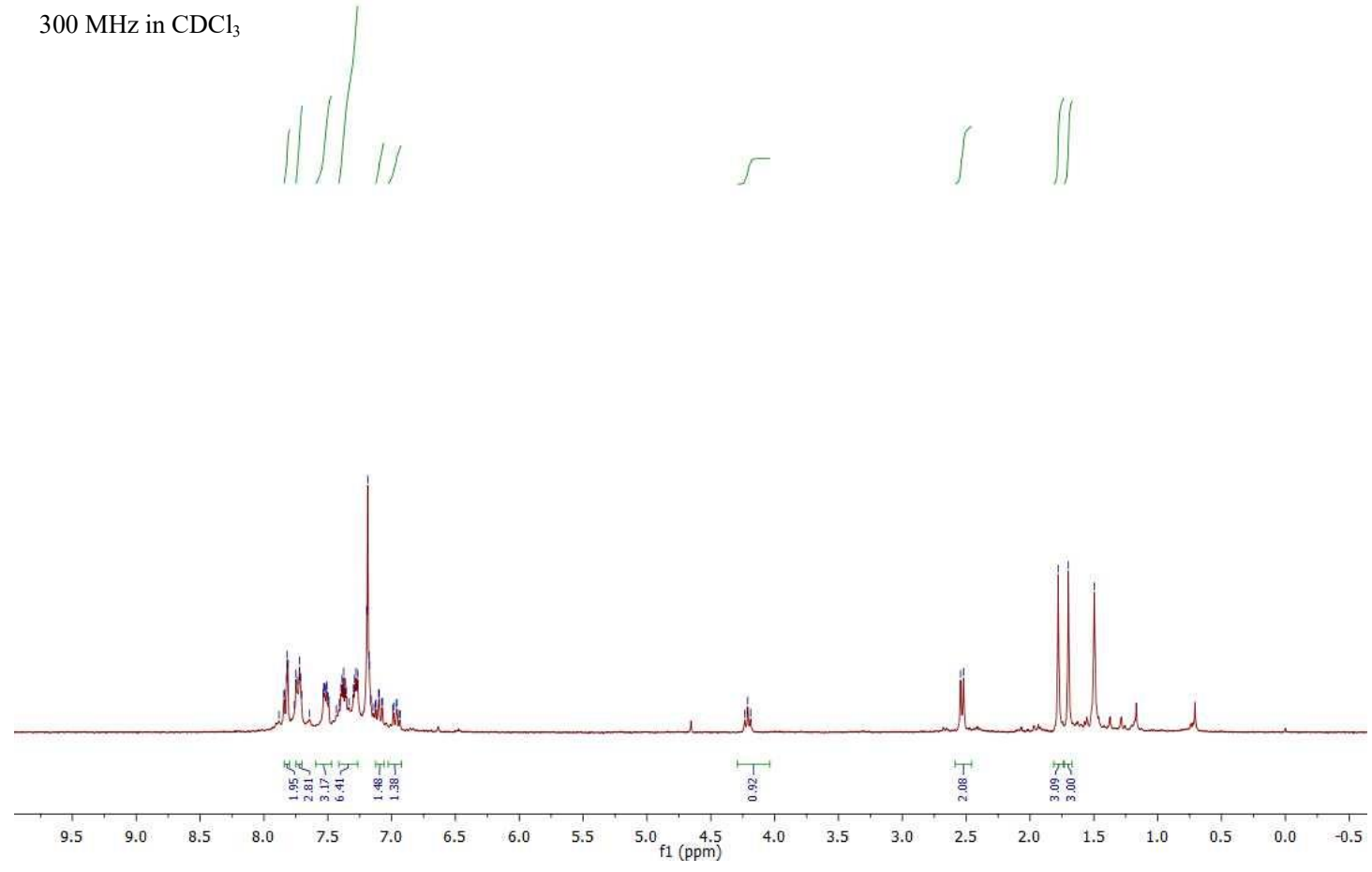

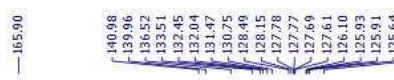

$151 \mathrm{MHz}$ in $\mathrm{CDCl}_{3}$

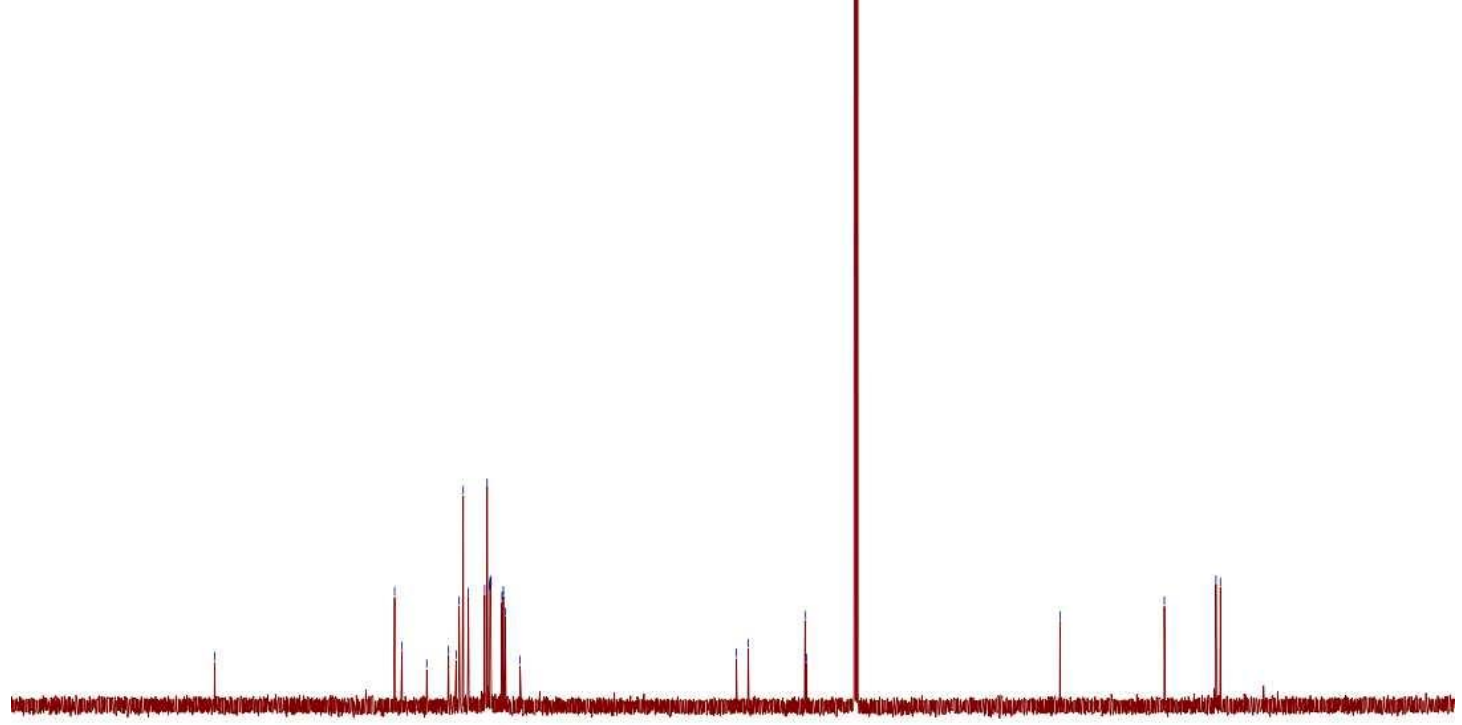

$\begin{array}{llllllllll}190 & 180 & 170 & 160 & 150 & 140 & 130 & 120 & 110 & 100 \\ \mathrm{f} 1(\mathrm{ppm})\end{array}$ 
<smiles>COc1ccc(CC(=O)OC(=O)c2ccccc2)cc1</smiles>

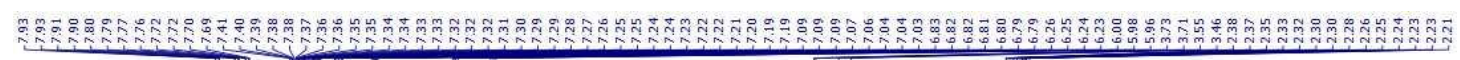
$300 \mathrm{MHz}$ in $\mathrm{CDCl}_{3}$

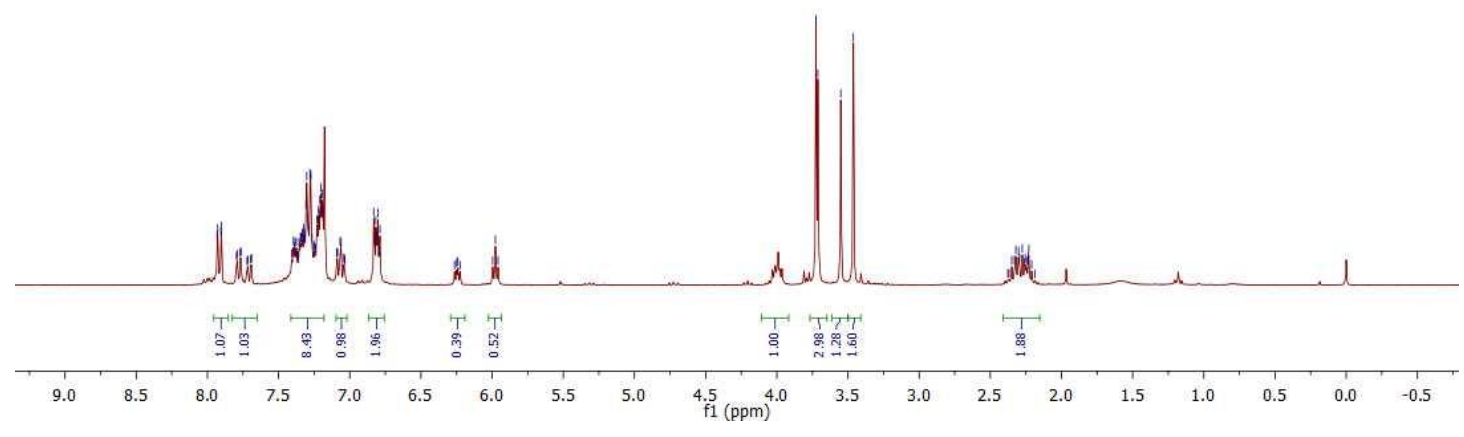

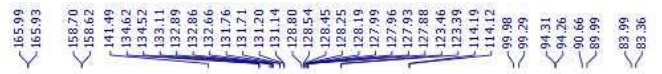
$76 \mathrm{MHz}$ in $\mathrm{CDCl}_{3}$

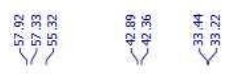

Mixture of diastereomer

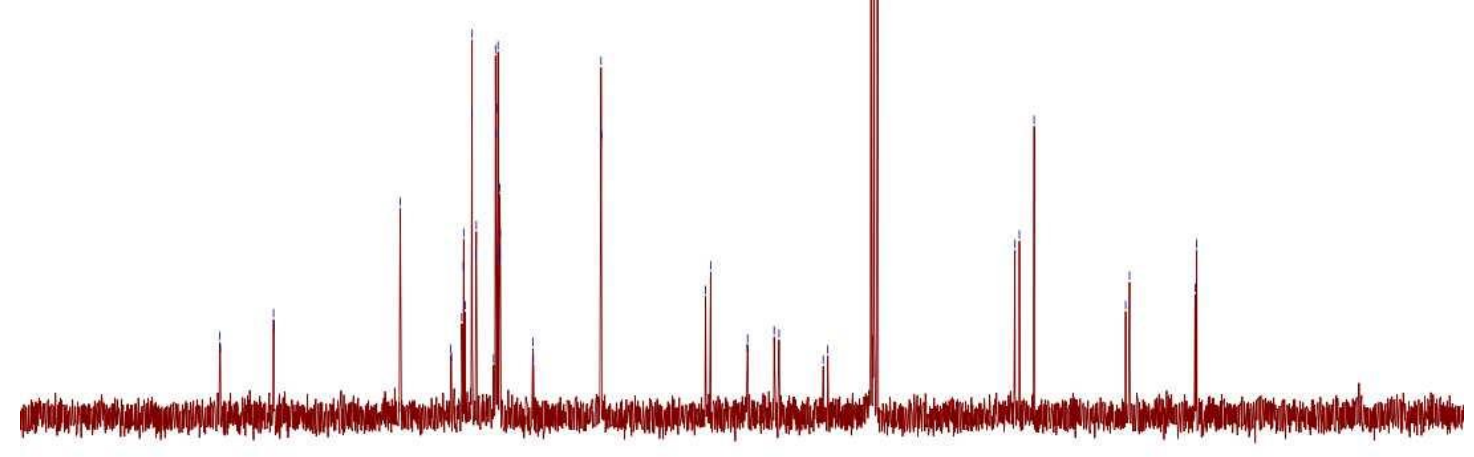

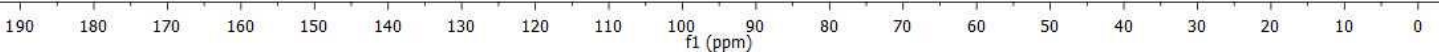


<smiles>COc1ccc(CC(CCN)C(=O)OC(=O)c2ccccc2)cc1</smiles>

(3p)

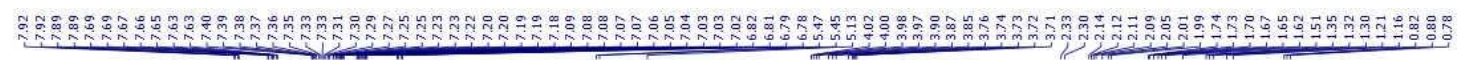
$300 \mathrm{MHz}$ in $\mathrm{CDCl}_{3}$

Mixture of diastereomer
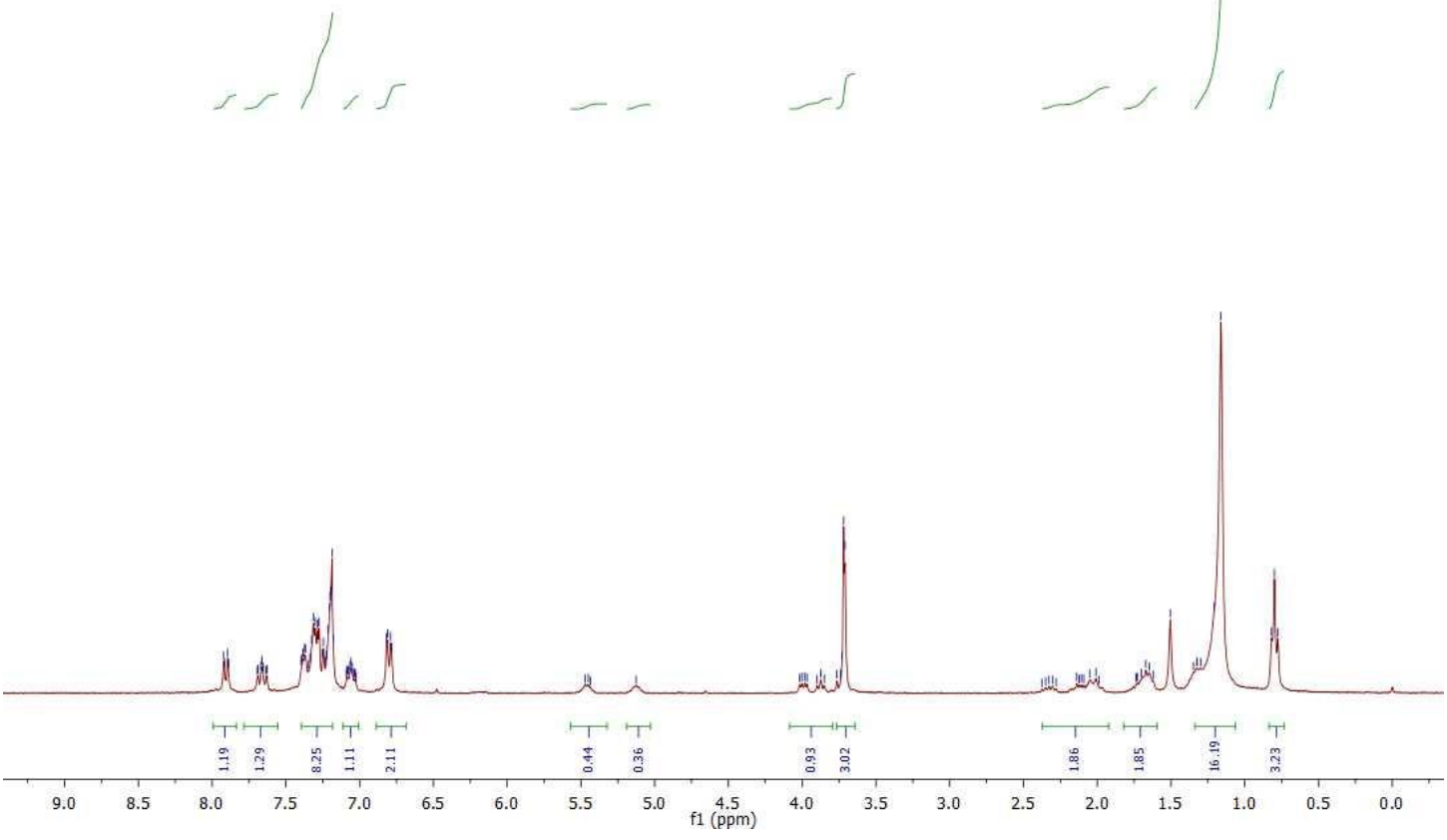

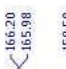

$151 \mathrm{MHz}$ in $\mathrm{CDCl}_{3}$

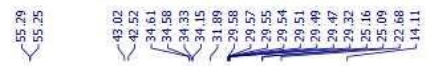

Mixture of diastereomer
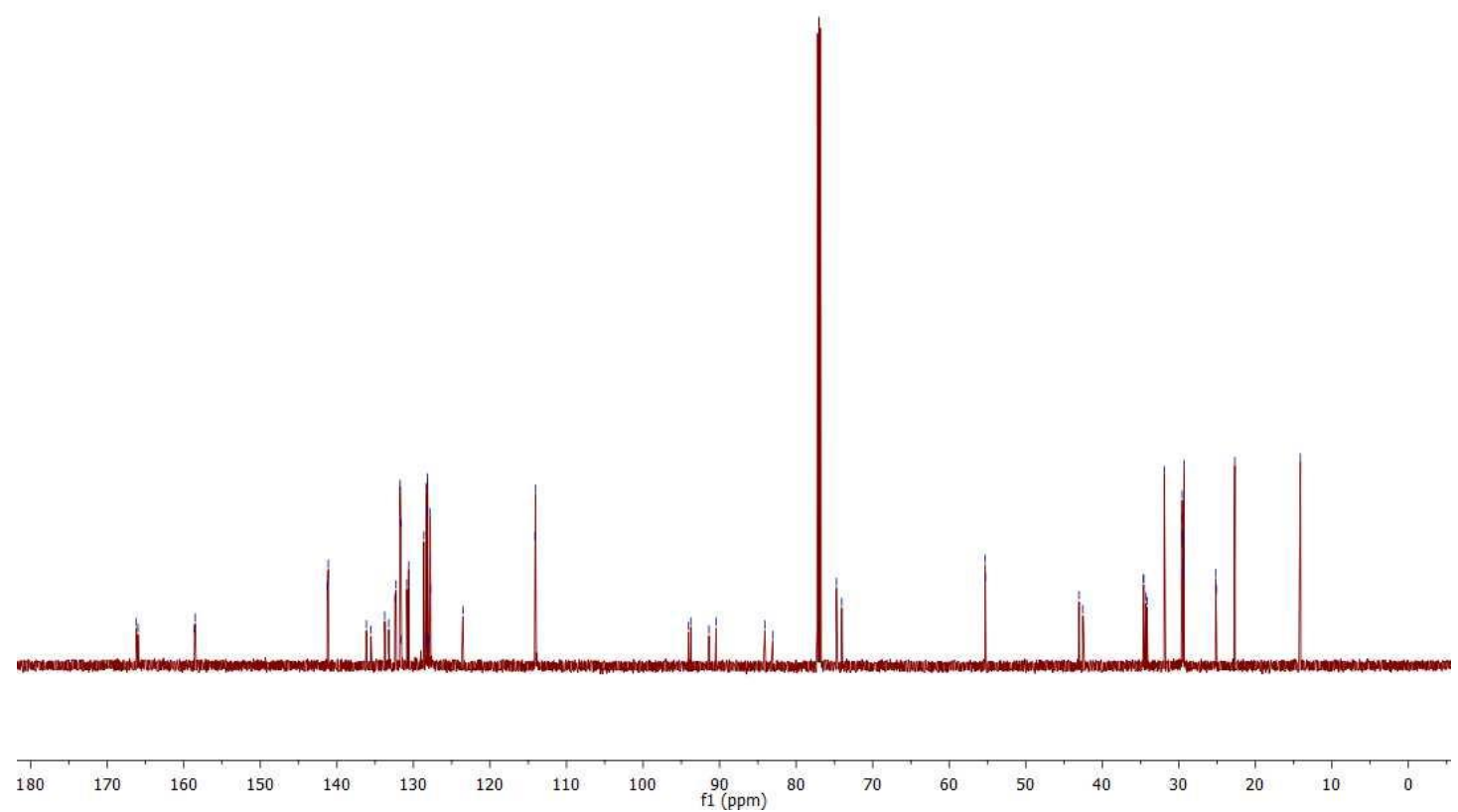

100

${ }_{10}^{90}(\mathrm{ppm})$

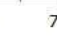

60 
<smiles>COc1ccc(C(C=Cc2ccccc2)CC(CCCO)OC(=O)c2ccccc2I)cc1</smiles>

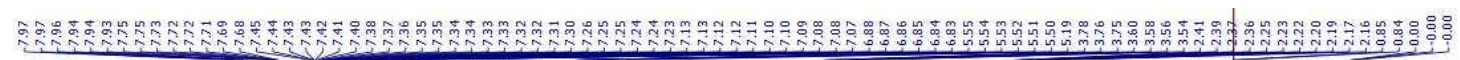
$300 \mathrm{MHz}$ in $\mathrm{CDCl}_{3}$

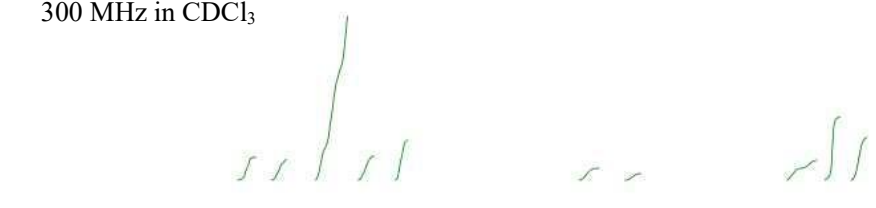

Mixture of diastereomer
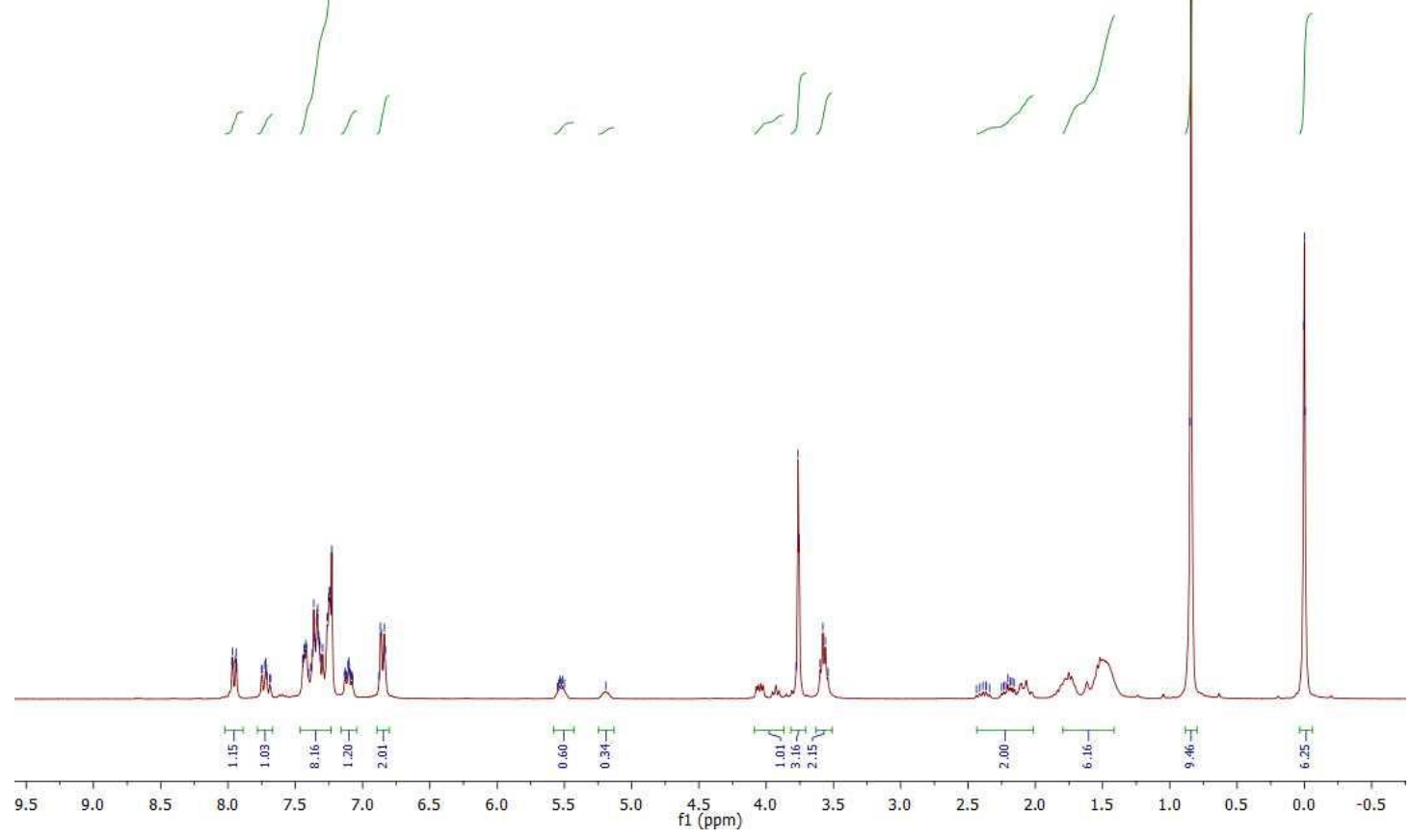

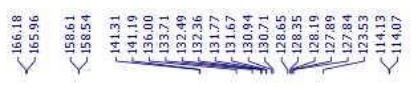

$76 \mathrm{MHz}$ in $\mathrm{CDCl}_{3}$

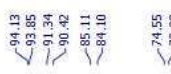

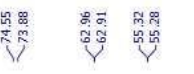

Mixture of diastereomer

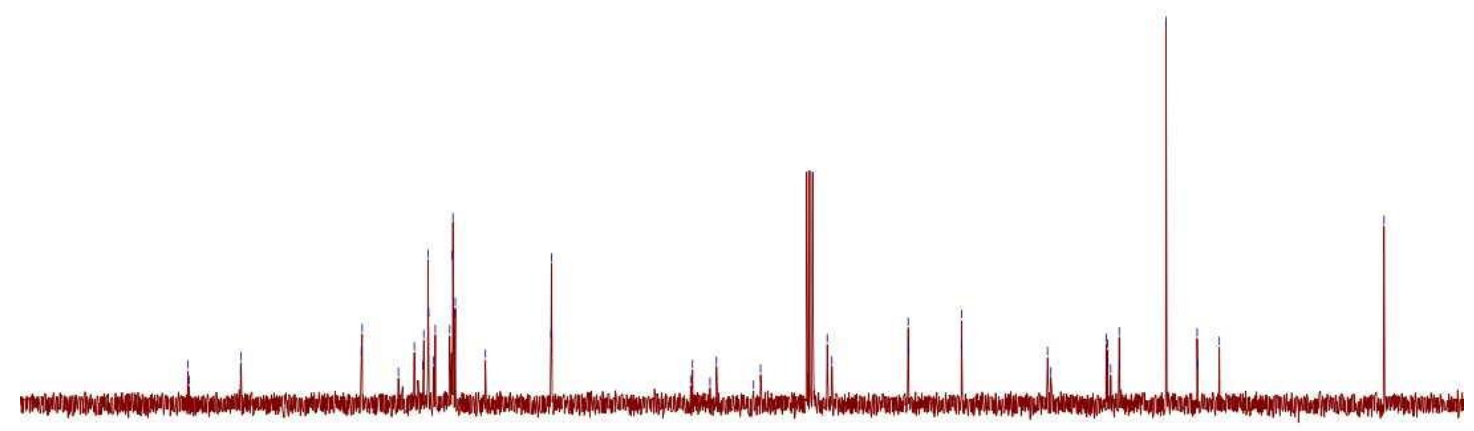

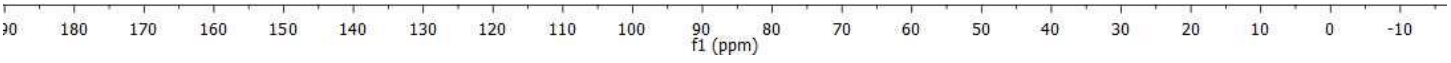




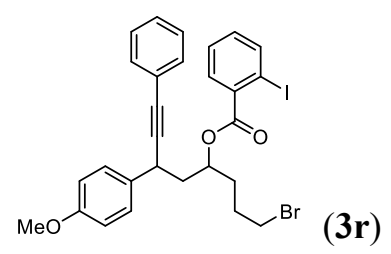

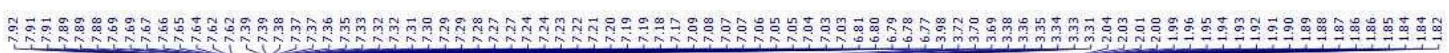
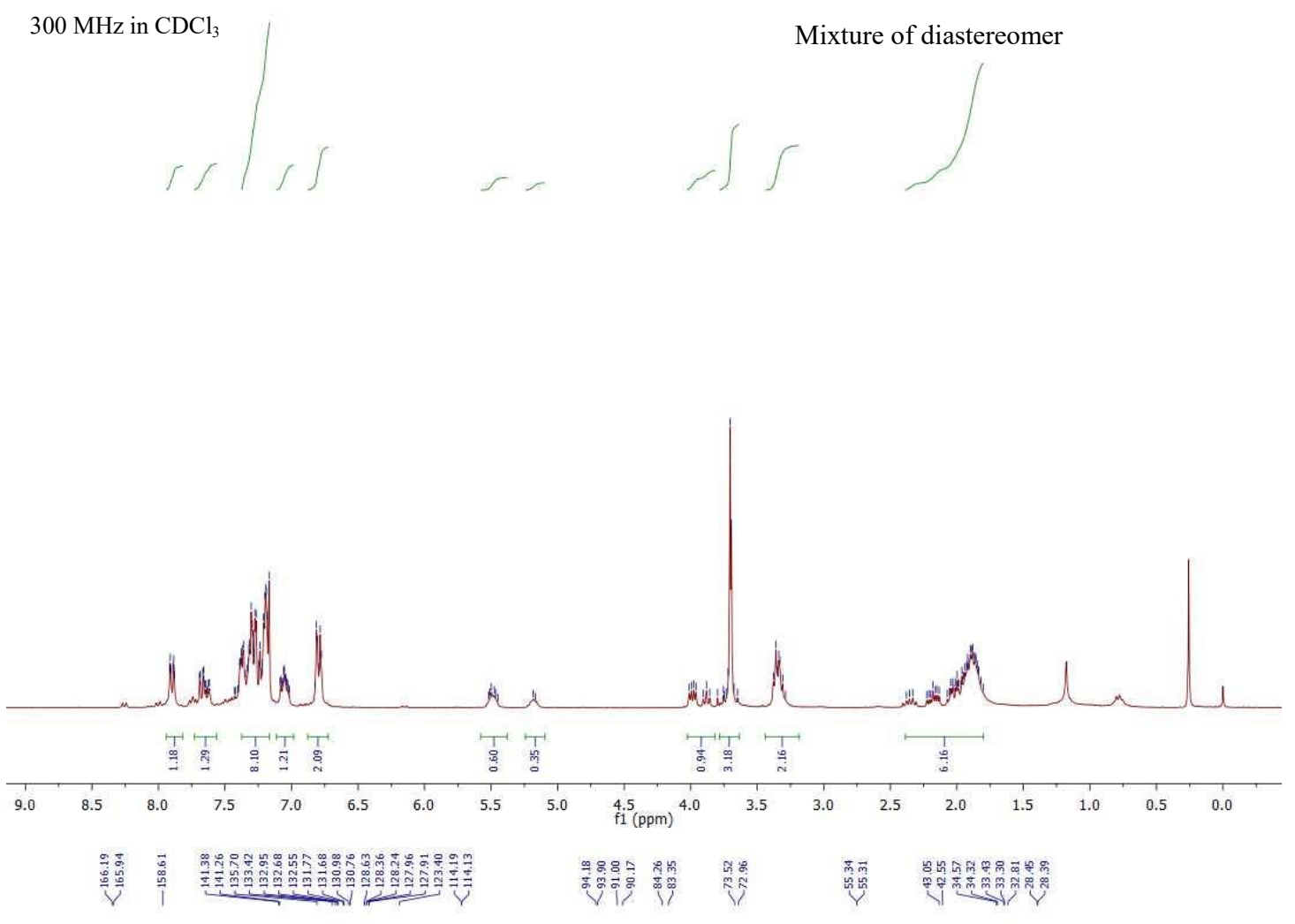

$76 \mathrm{MHz}$ in $\mathrm{CDCl}_{3}$

Mixture of diastereomer
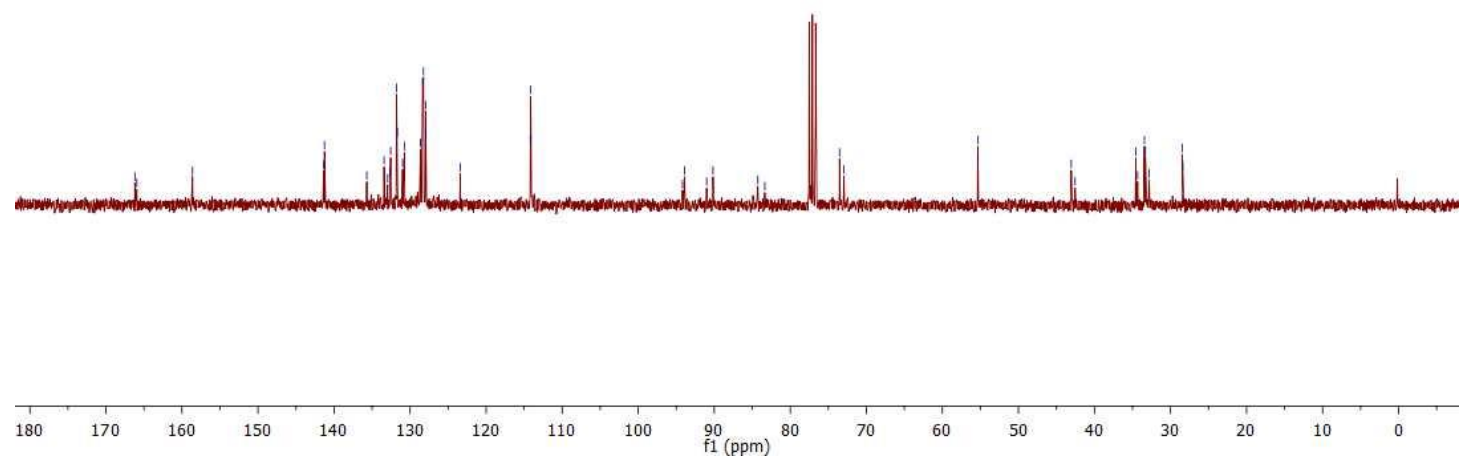

S48 


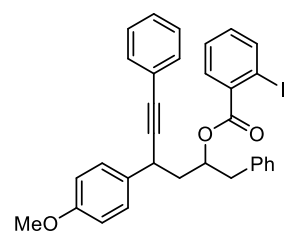

(3s)

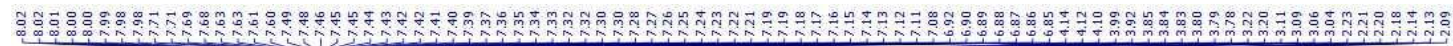

$300 \mathrm{MHz}$ in $\mathrm{CDCl}_{3}$

Mixture of diastereomer

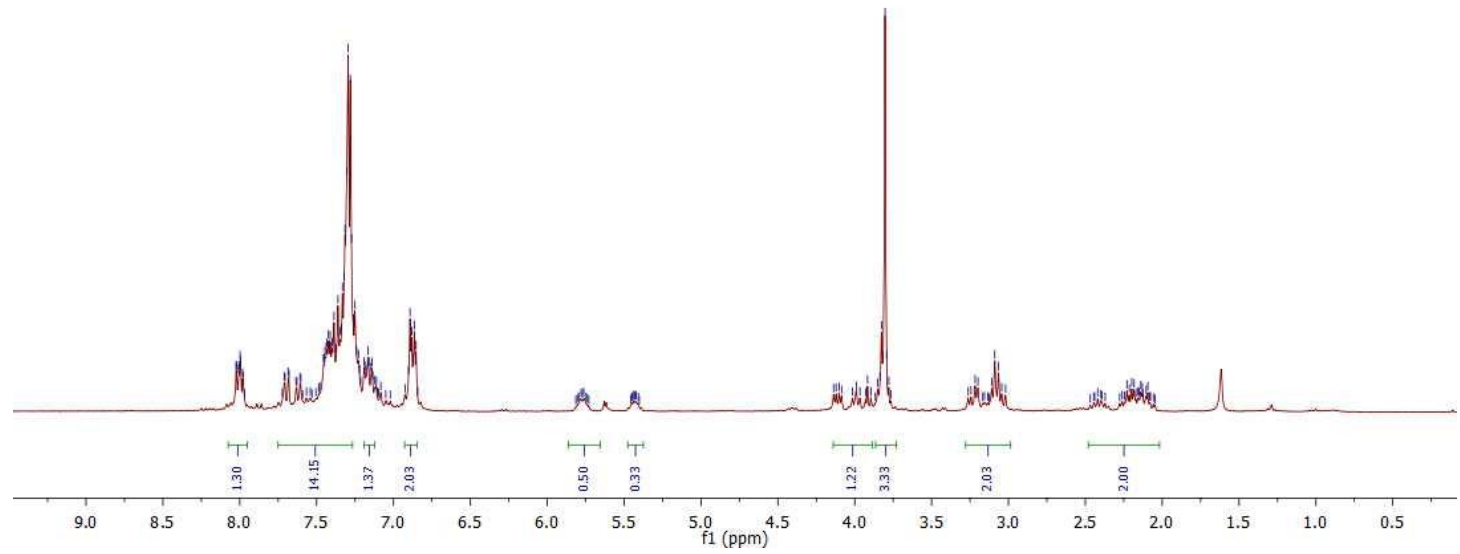

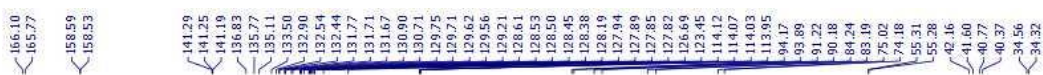

$76 \mathrm{MHz}$ in $\mathrm{CDCl}_{3}$

Mixture of diastereomer

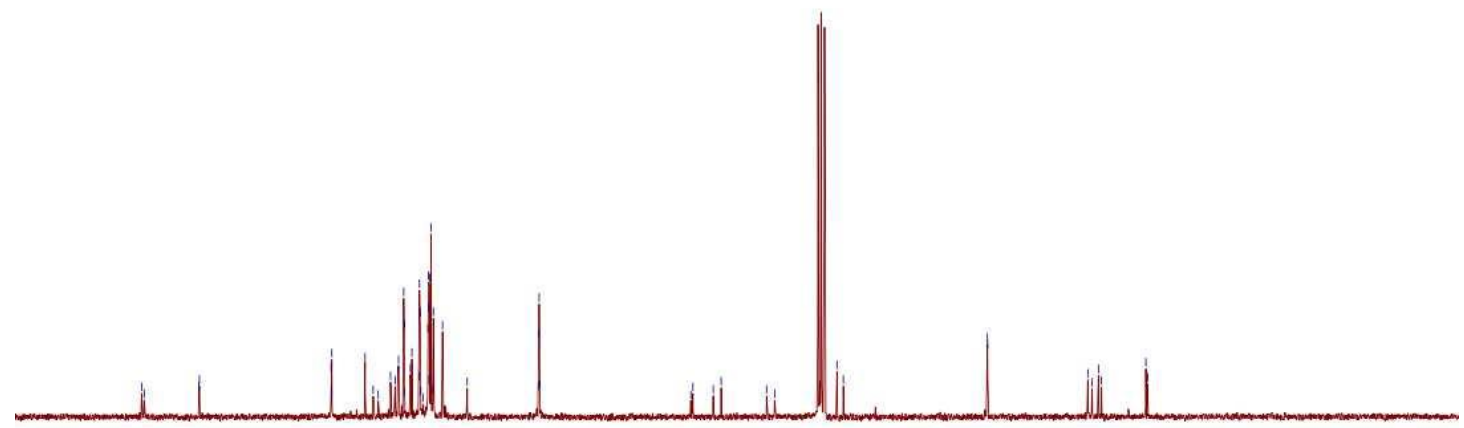

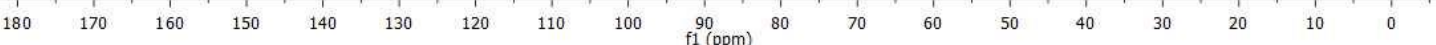




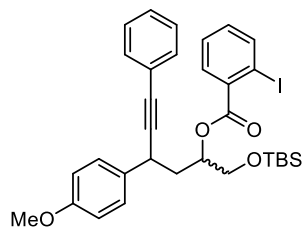

(3t)

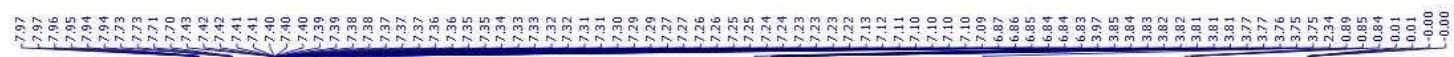
$300 \mathrm{MHz}$ in $\mathrm{CDCl}_{3}$

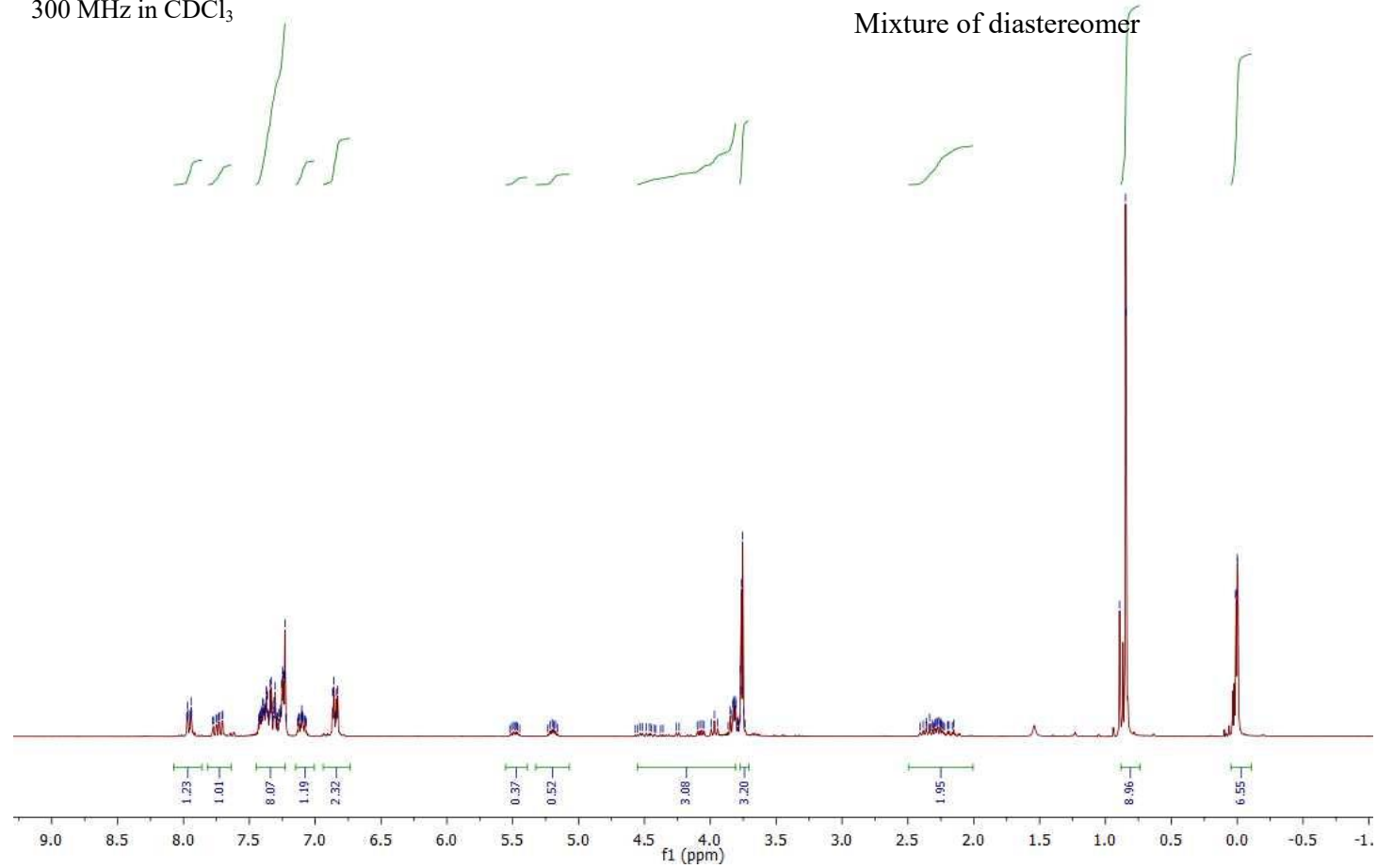

Mixture of diastereomer

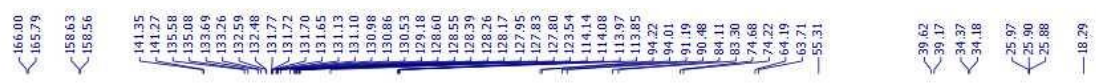

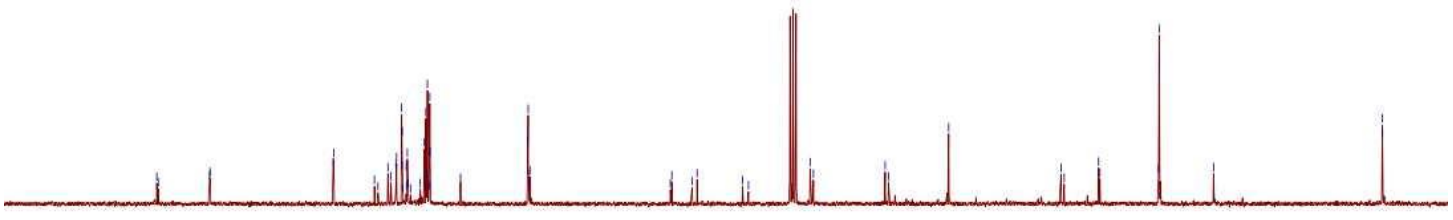

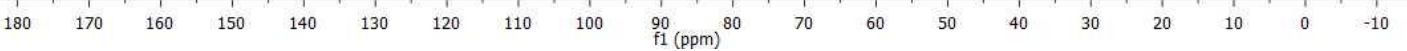




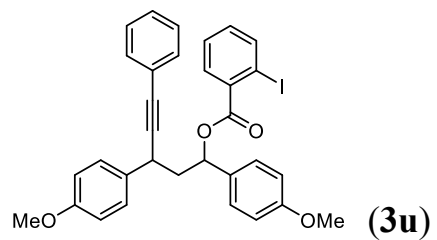

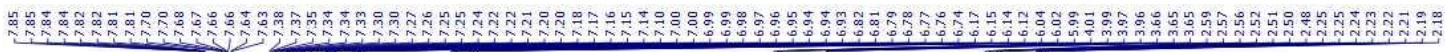

$300 \mathrm{MHz}$ in $\mathrm{CDCl}_{3}$

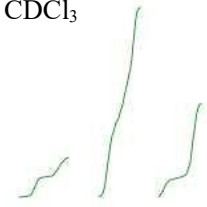

Mixture of diastereomer

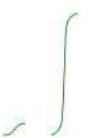

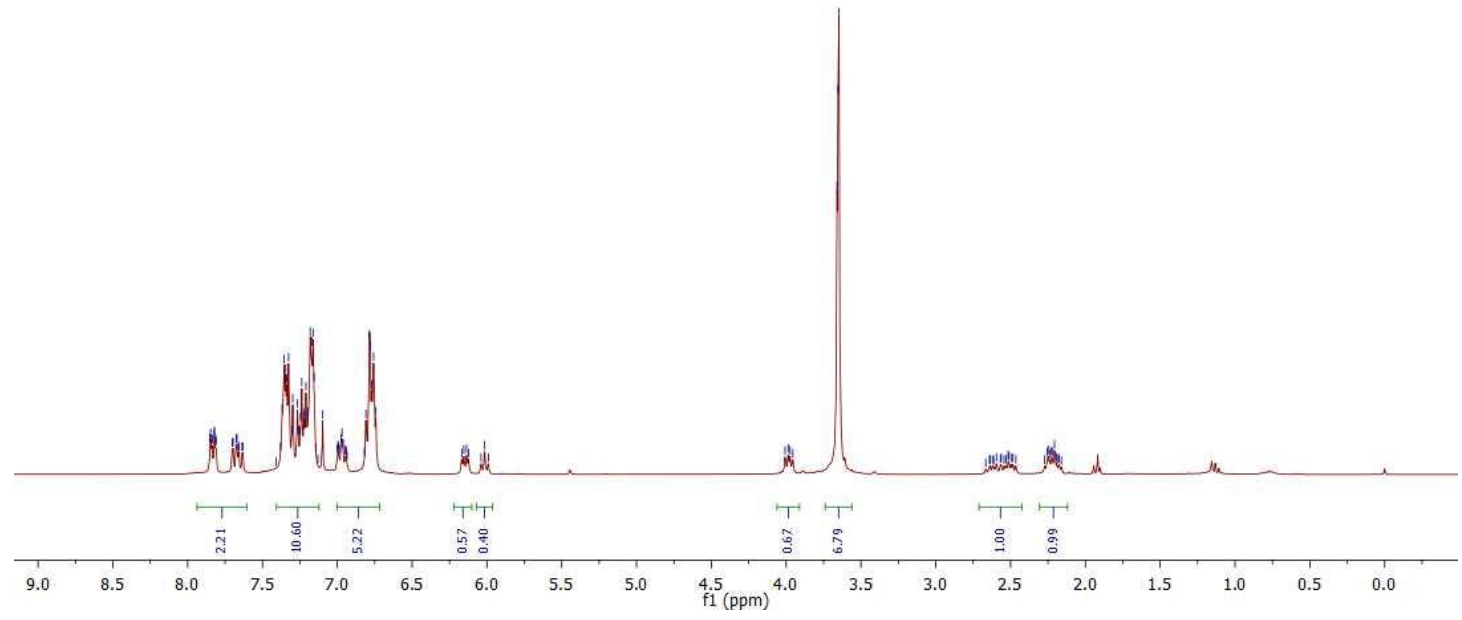

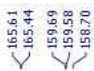

$76 \mathrm{MHz}$ in $\mathrm{CDCl}_{3}$

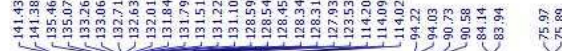

Mixture of diastereomer

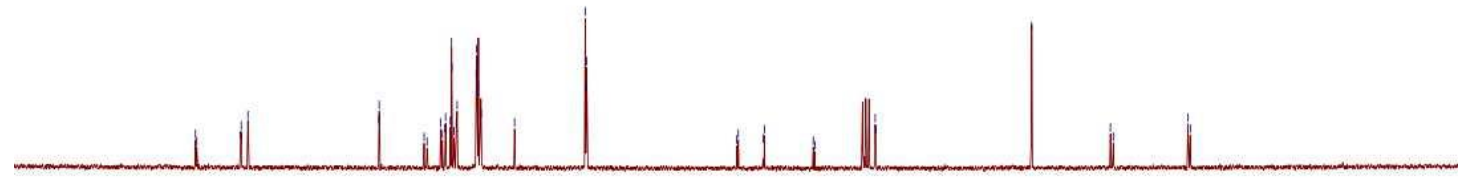

$\begin{array}{lllllllll}180 & 170 & 160 & 150 & 140 & 130 & 120 & 110 & 100 \\ \mathrm{f} 1(\mathrm{ppm})\end{array}$ 


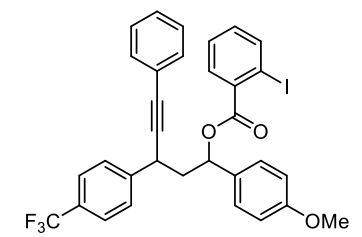

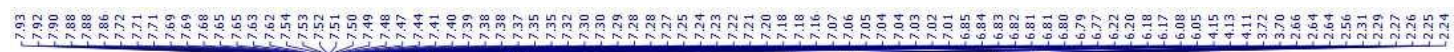

$300 \mathrm{MHz}$ in $\mathrm{CDCl}_{3}$

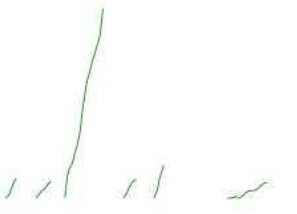

Mixture of diastereomer and regioisomer

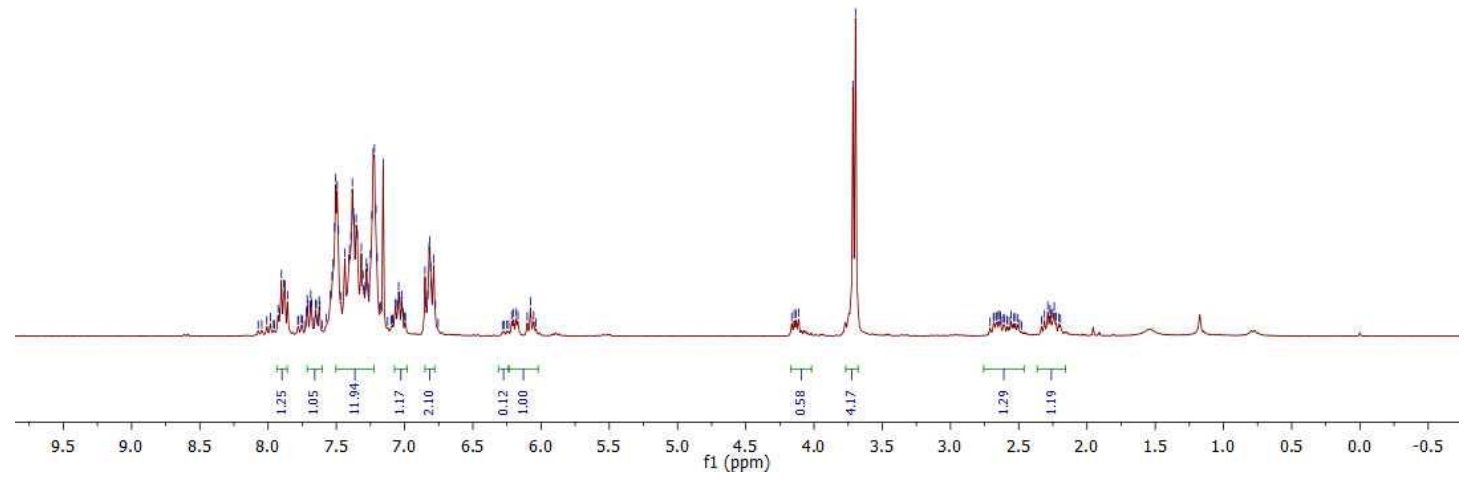

㺟聚?

$282 \mathrm{MHz}$ in $\mathrm{CDCl}_{3}$

Mixture of diastereomer and regioisomer

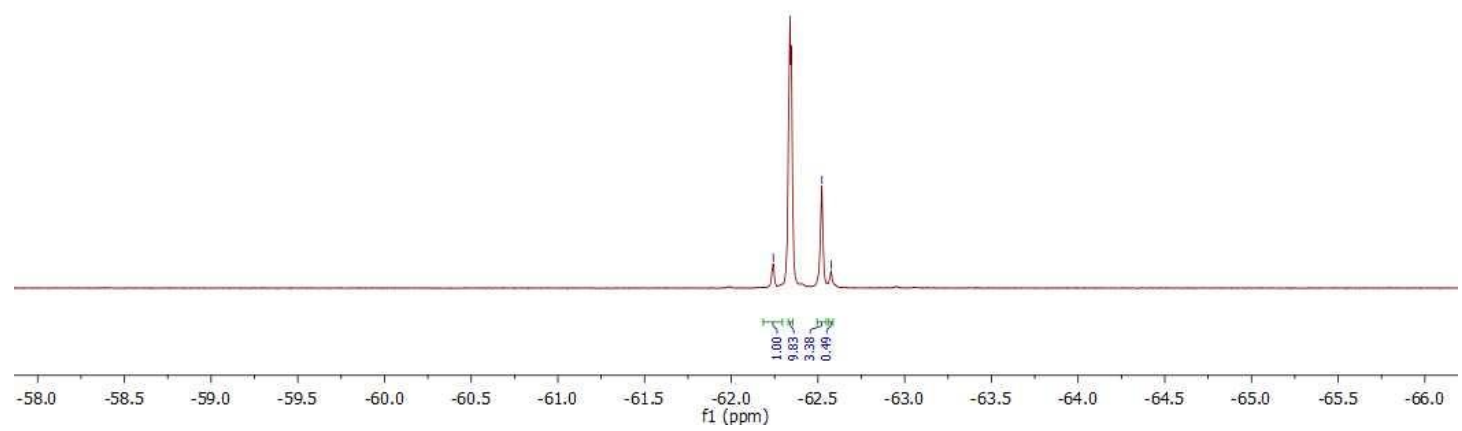




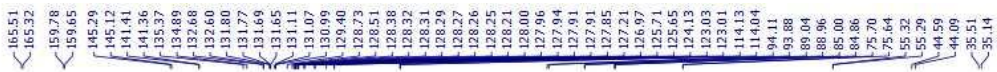

$151 \mathrm{MHz}$ in $\mathrm{CDCl}_{3}$

Mixture of diastereomer and regioisomer

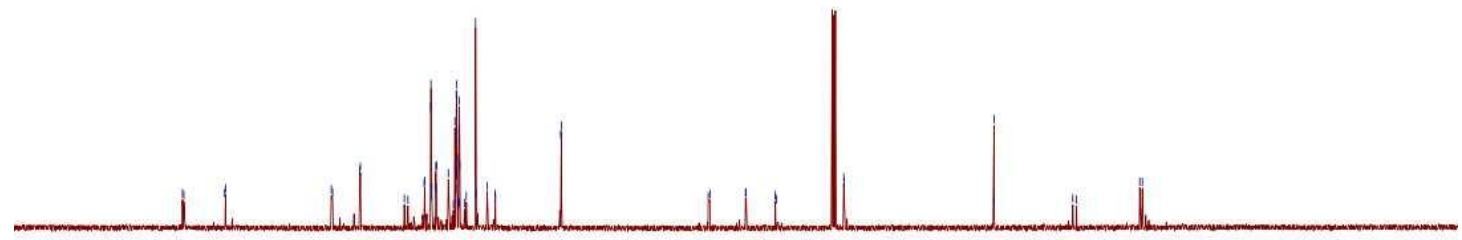

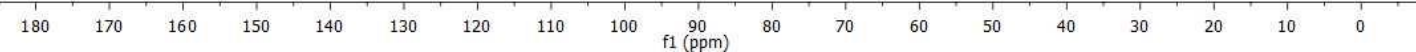




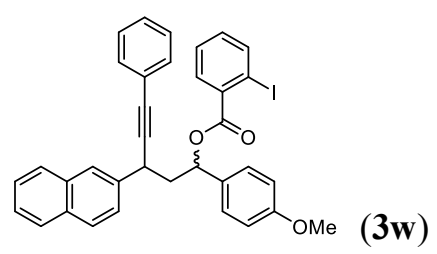

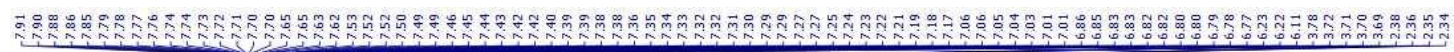

$300 \mathrm{MHz}$ in $\mathrm{CDCl}_{3}$

Mixture of diastereomer and regioisomer
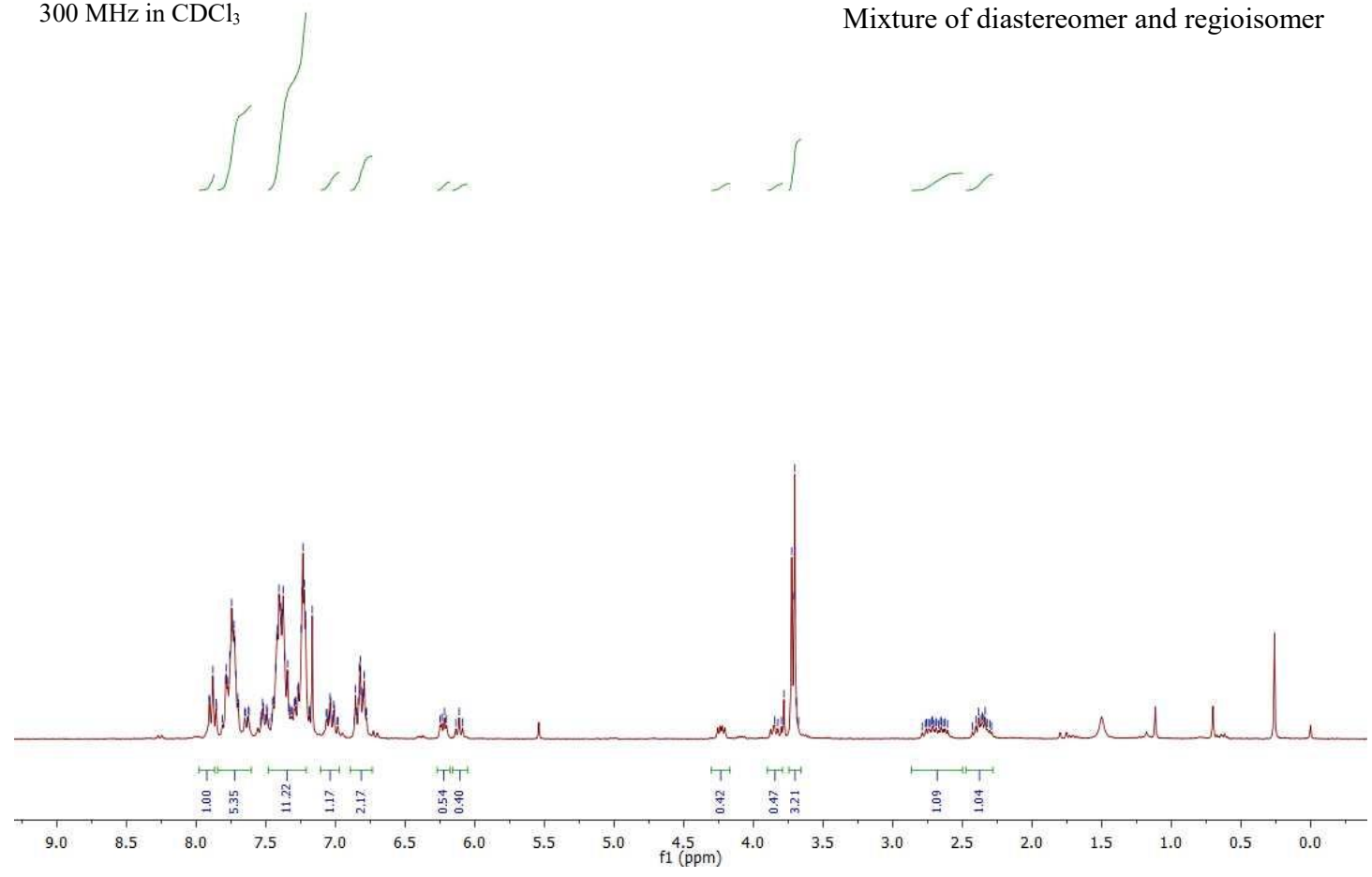

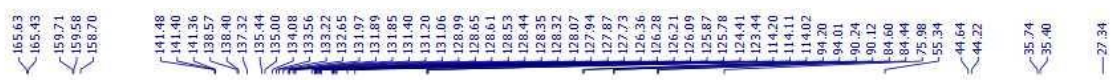

$76 \mathrm{MHz}$ in $\mathrm{CDCl}_{3}$

Mixture of diastereomer and regioisomer

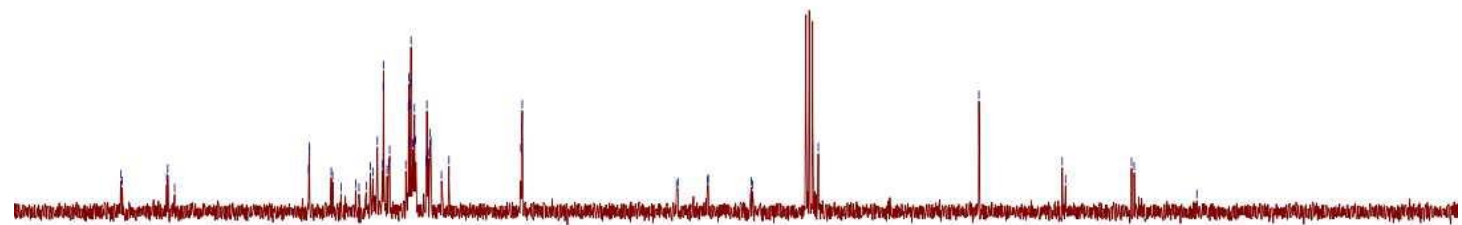

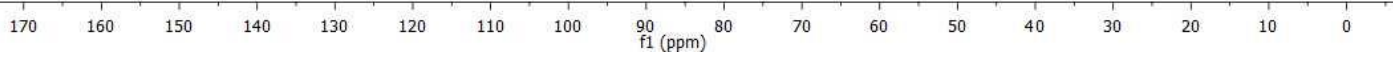




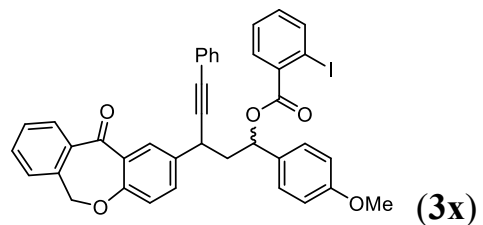

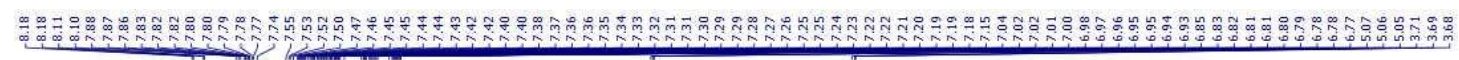

$300 \mathrm{MHz}$ in $\mathrm{CDCl}_{3}$

Mixture of diastereomer and regioisomer
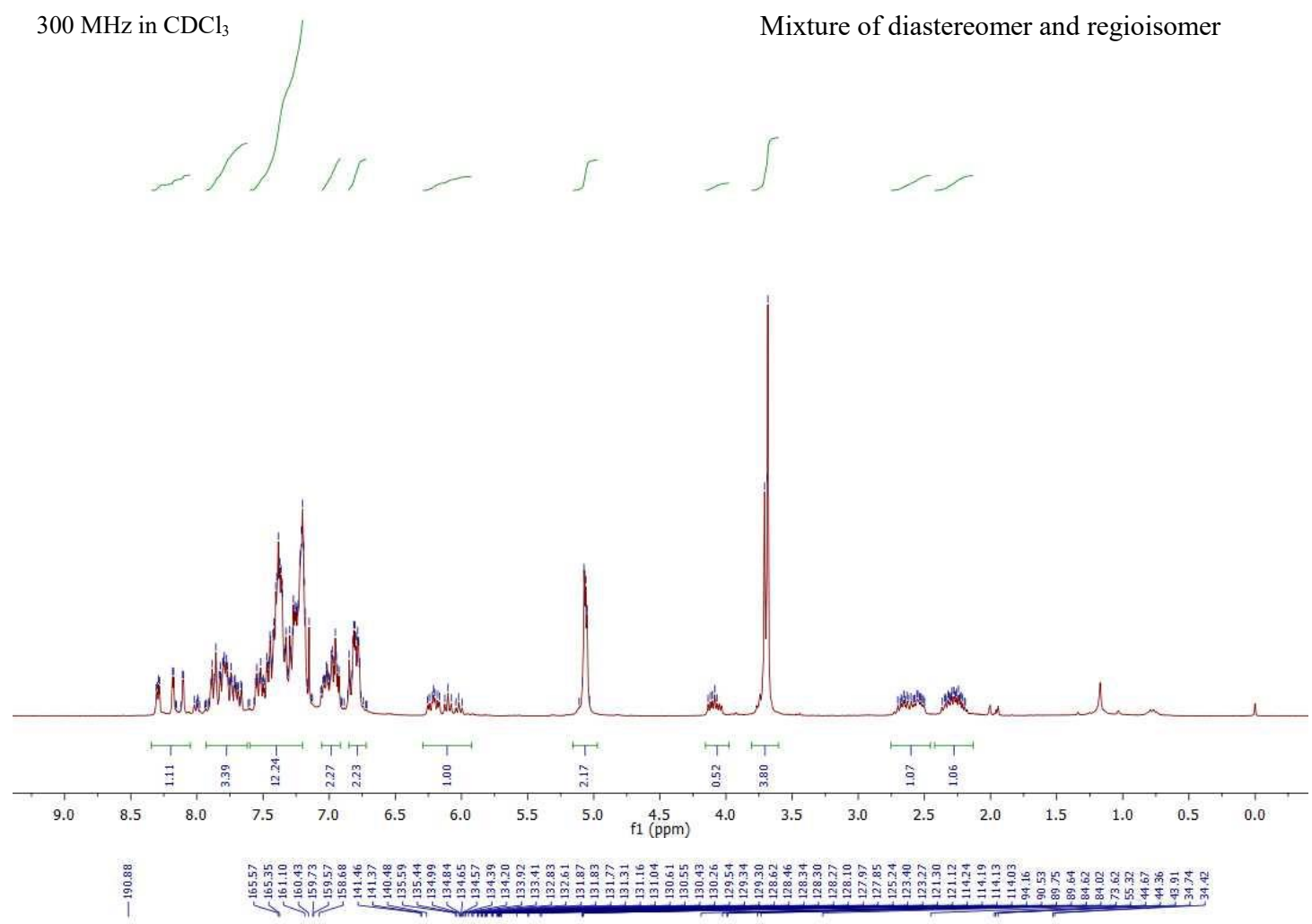

$76 \mathrm{MHz}$ in $\mathrm{CDCl}_{3}$

Mixture of diastereomer and regioisomer

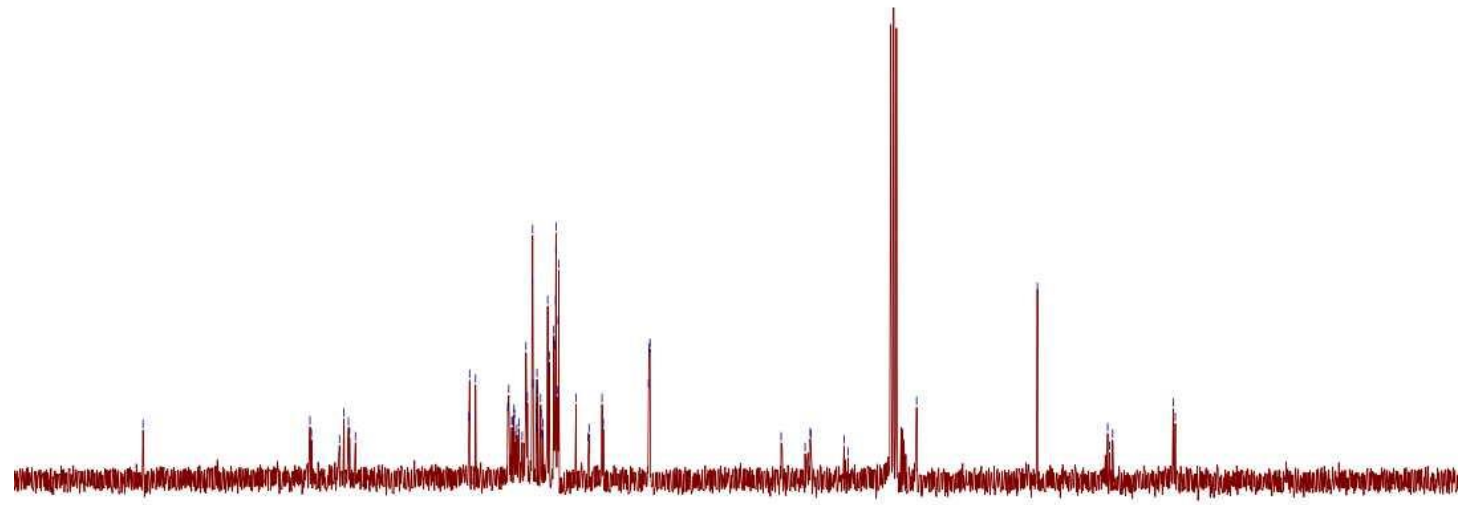

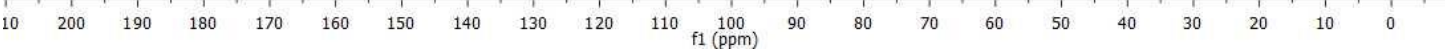




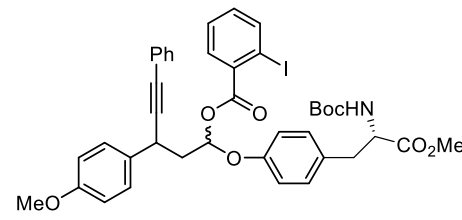

(3y)

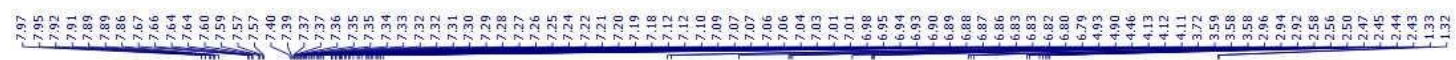

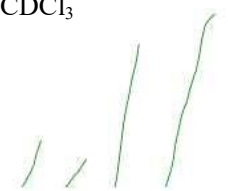

$300 \mathrm{MHz}$ in $\mathrm{CDCl}$

Mixture of diastereomer
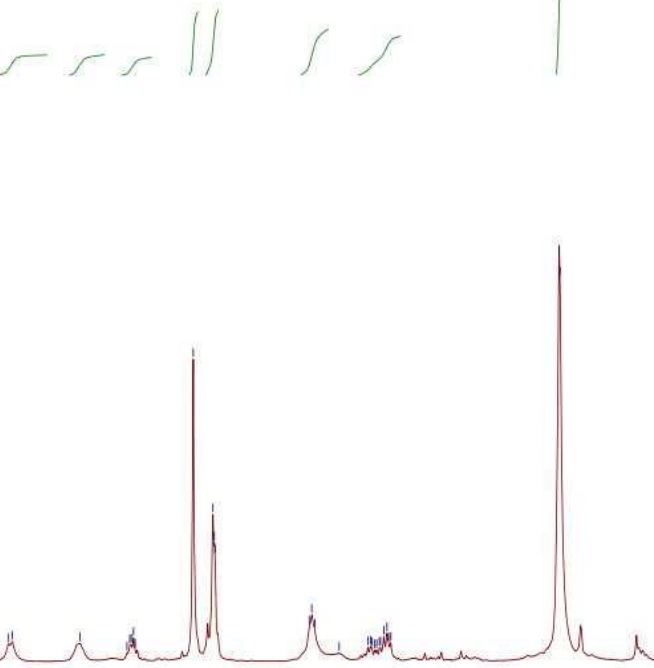

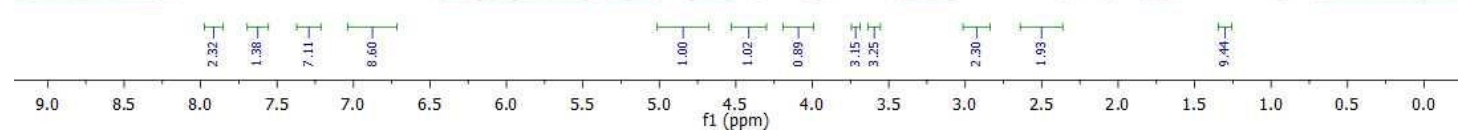

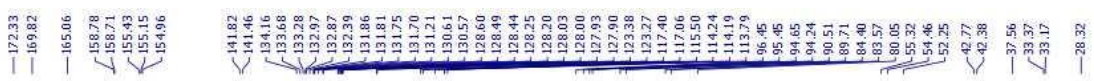

$76 \mathrm{MHz}$ in $\mathrm{CDCl}_{3}$

Mixture of diastereomer and regioisomer

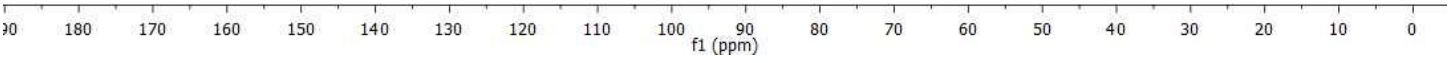




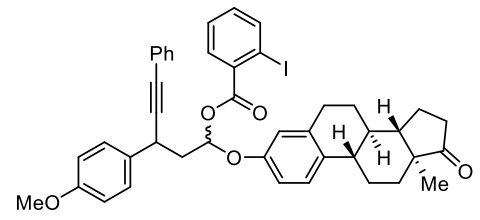

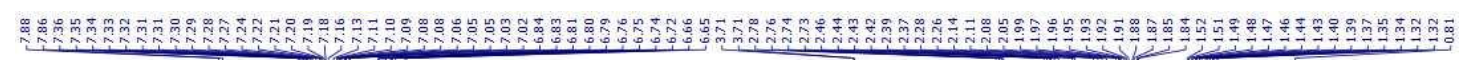

$300 \mathrm{MHz}$ in $\mathrm{CDCl}_{3}$

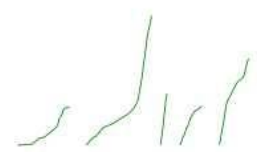

Mixture of diastereomer and regioisomer

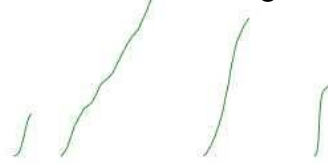

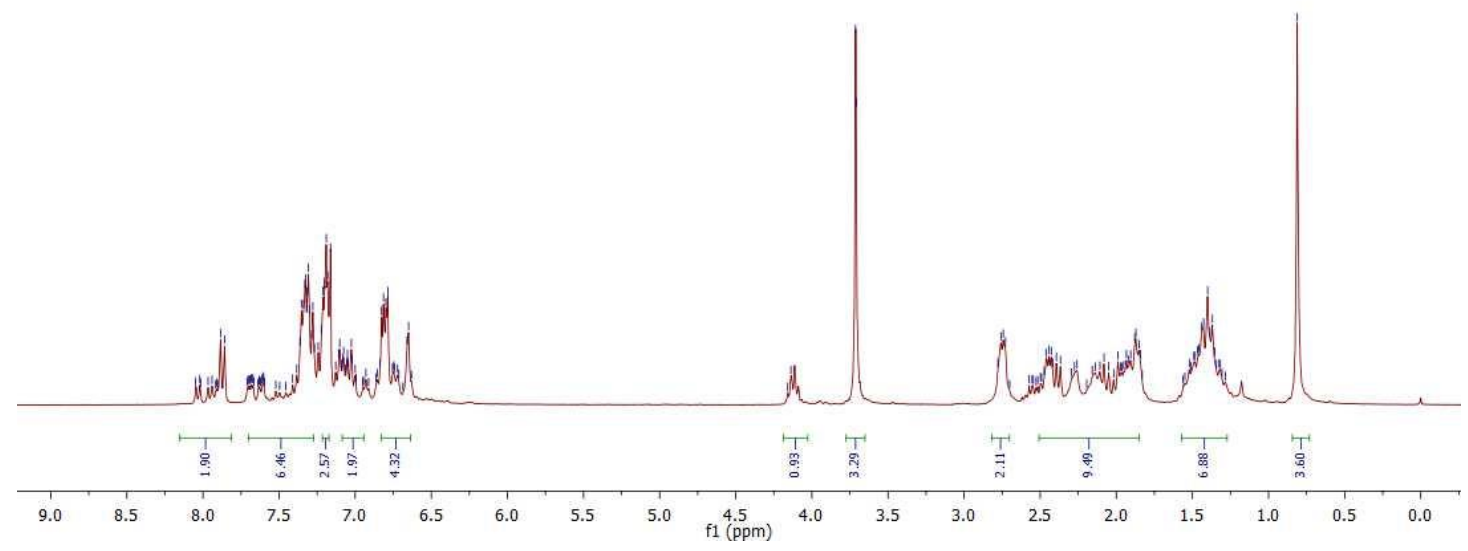

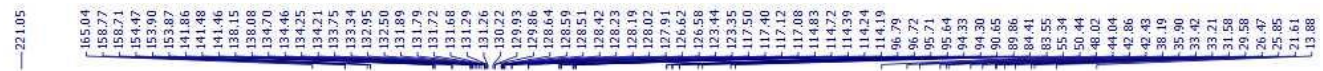

$76 \mathrm{MHz}$ in $\mathrm{CDCl}_{3}$

Mixture of diastereomer and regioisomer

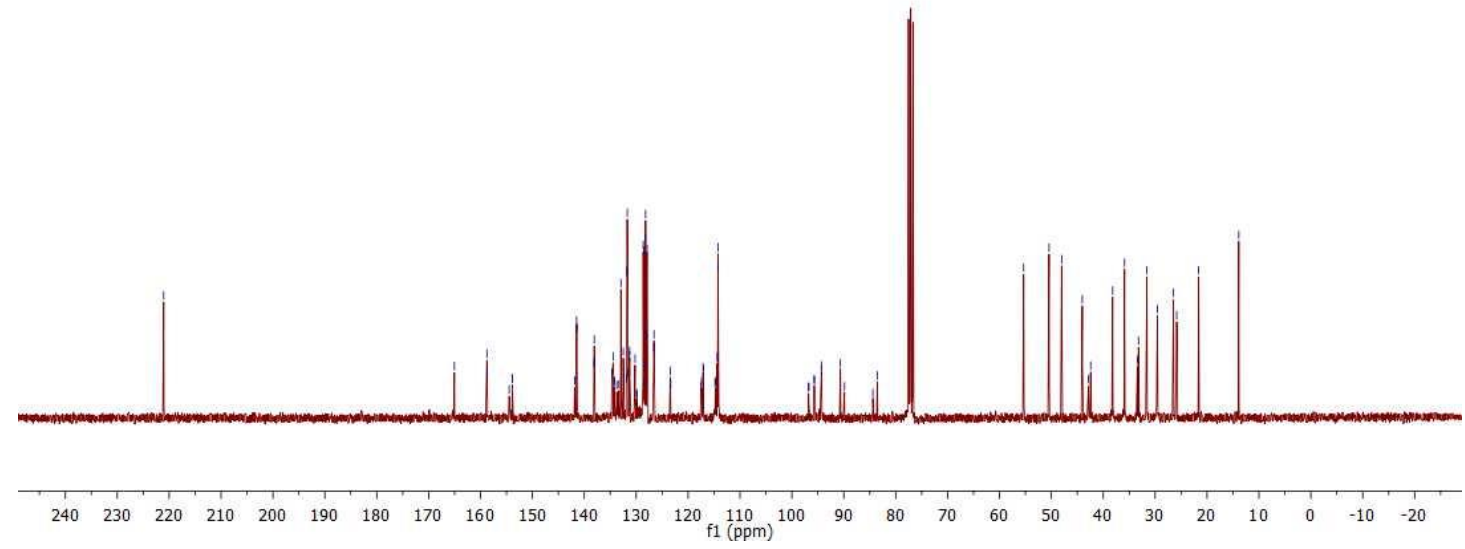




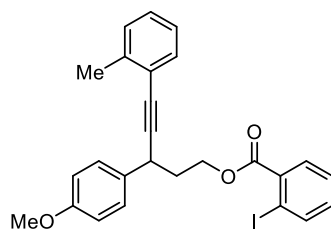

(3aa)

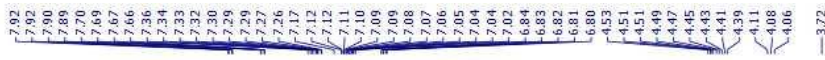

$300 \mathrm{MHz}$ in $\mathrm{CDCl}_{3}$
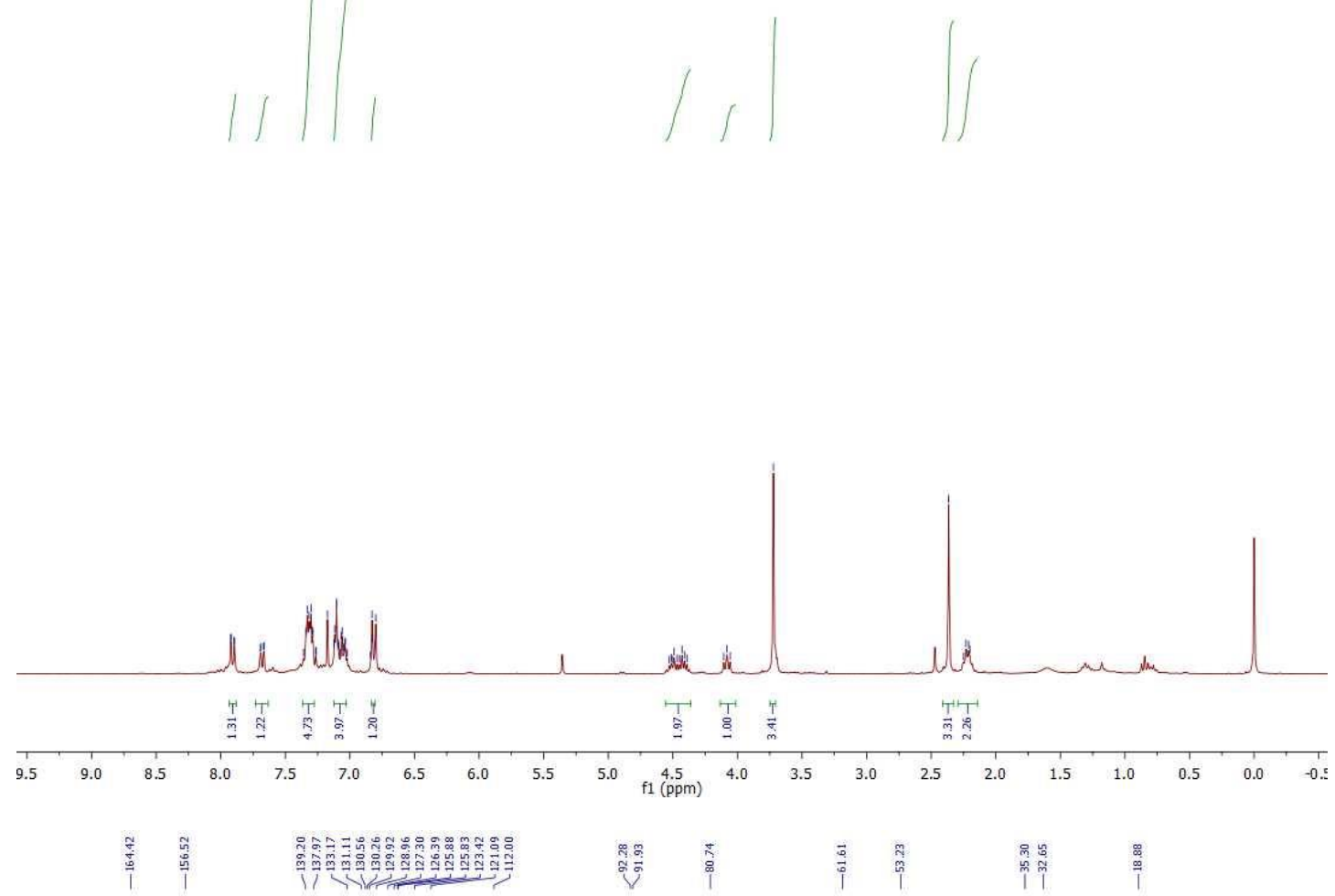

$76 \mathrm{MHz}$ in $\mathrm{CDCl}_{3}$

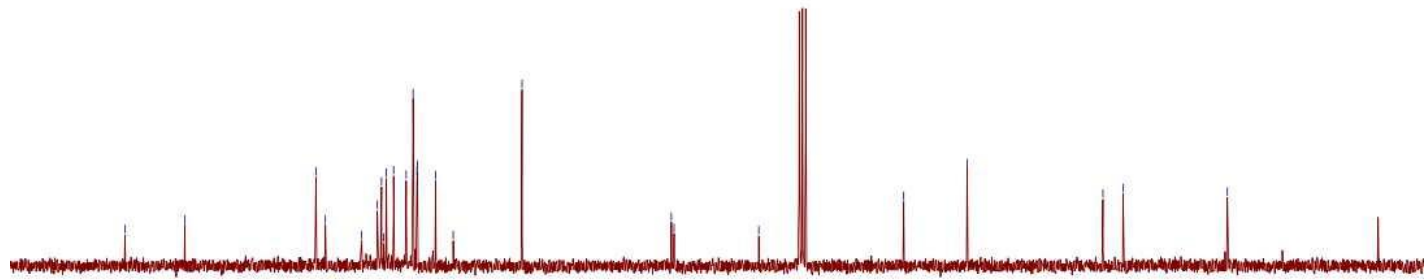

$\begin{array}{lllllllll}170 & 160 & 150 & 140 & 130 & 120 & 110 & 100 & \underset{\mathrm{f} 1(\mathrm{ppm})}{90}\end{array}$ 


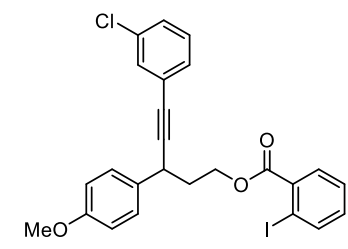

(3ab)

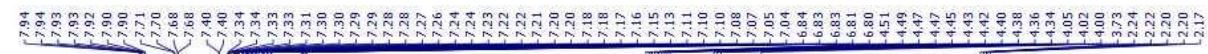

$300 \mathrm{MHz}$ in $\mathrm{CDCl}_{3}$
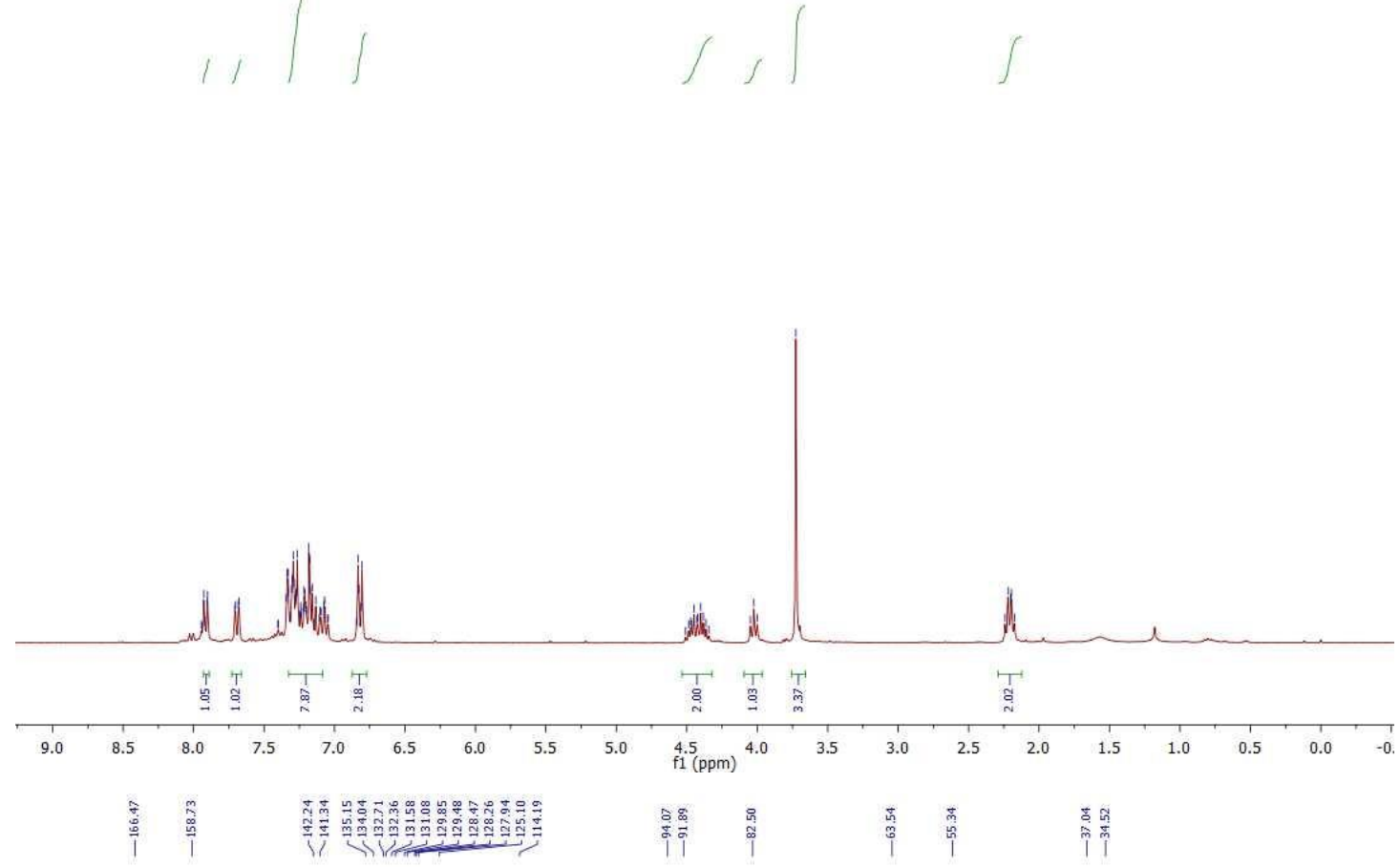

$76 \mathrm{MHz}$ in $\mathrm{CDCl}_{3}$
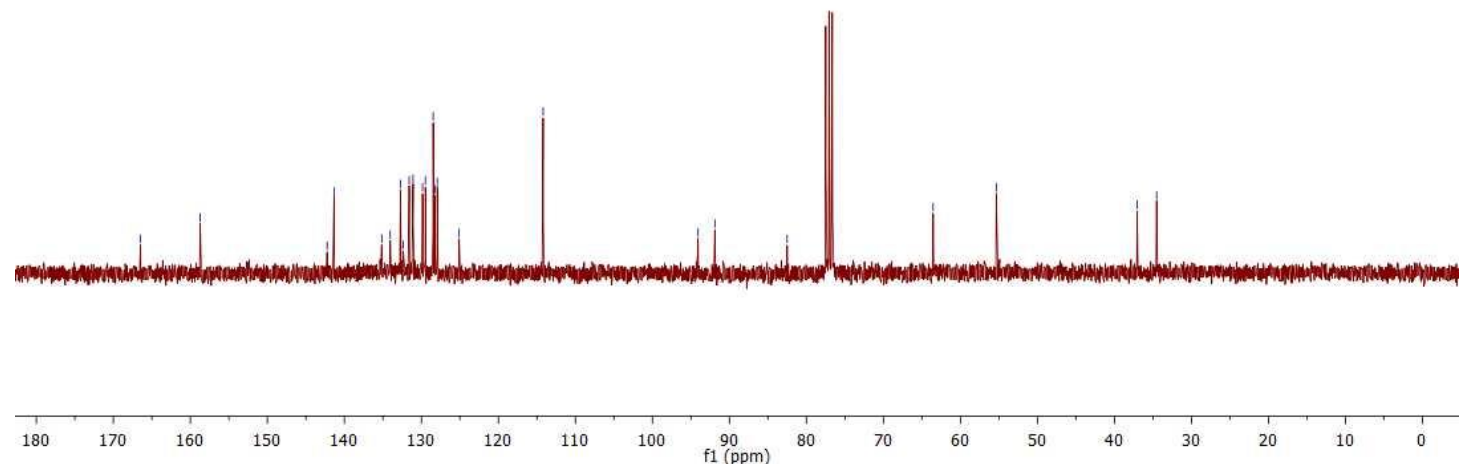


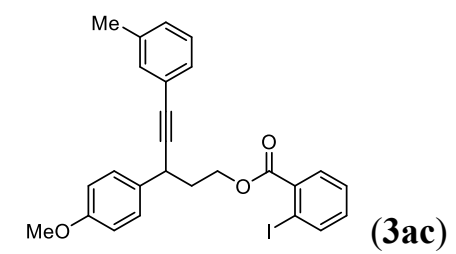

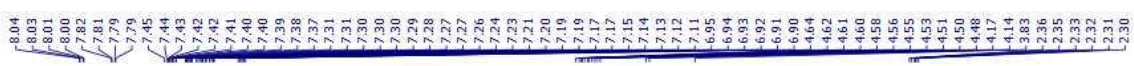

$300 \mathrm{MHz}$ in $\mathrm{CDCl}_{3}$
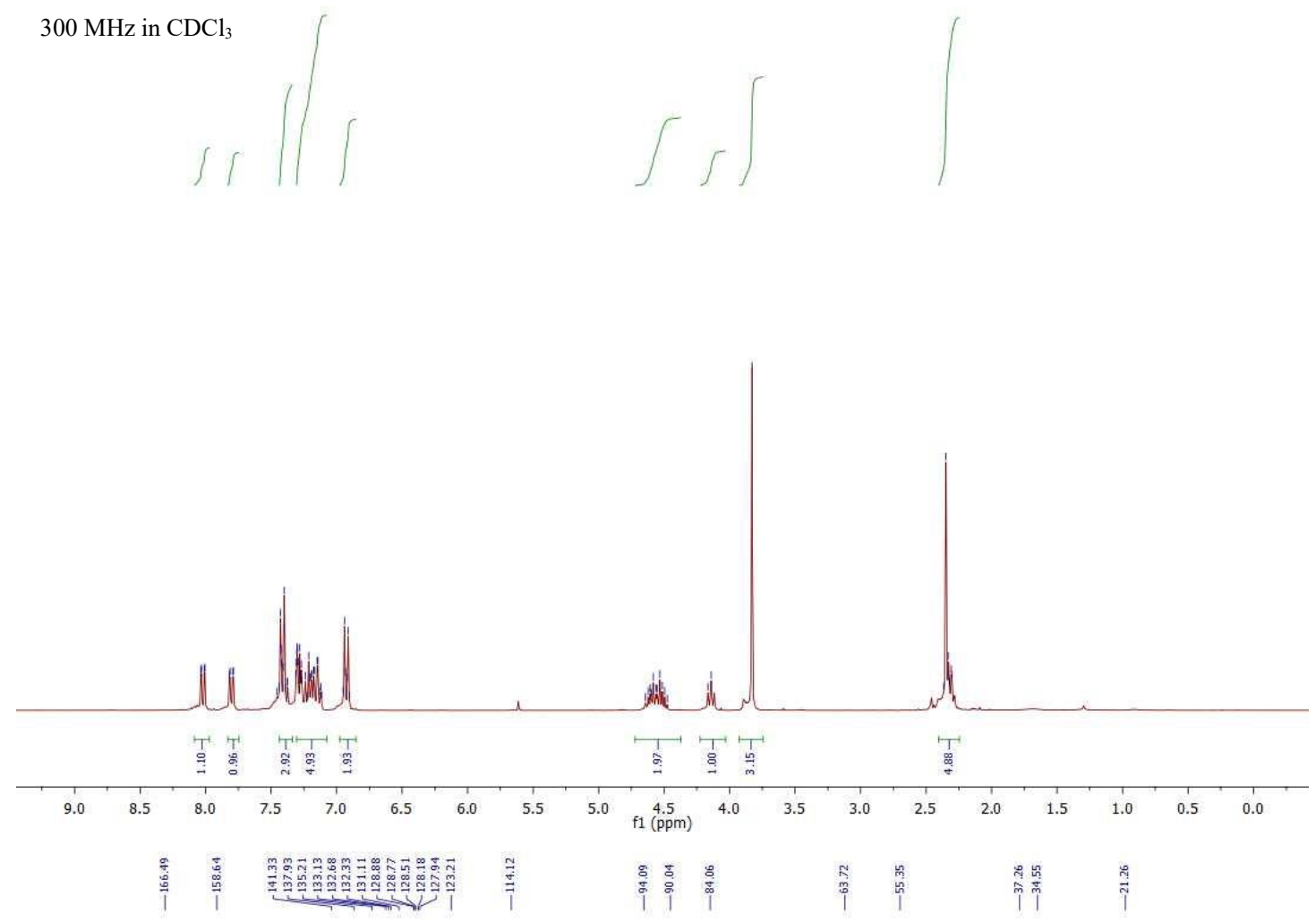

$76 \mathrm{MHz}$ in $\mathrm{CDCl}_{3}$

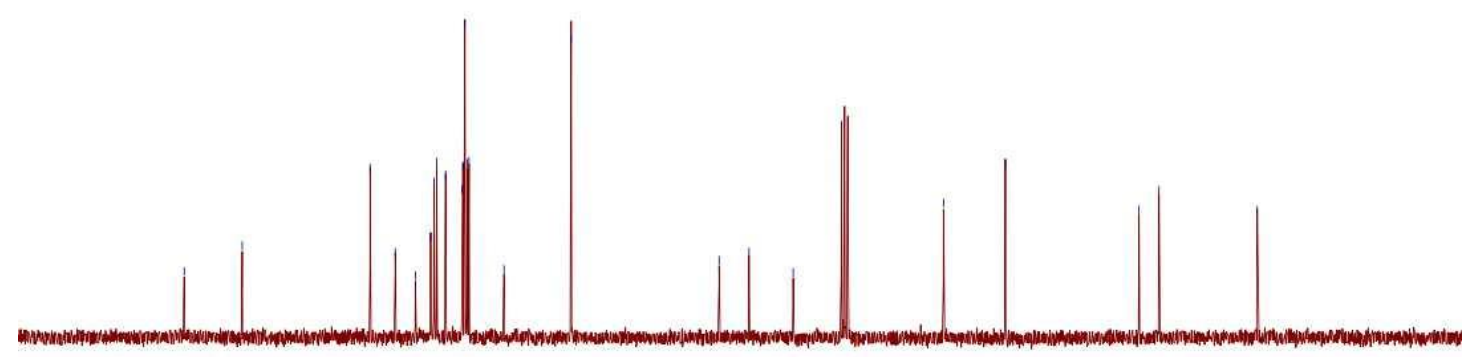

\begin{tabular}{lllllllllllllllll}
1 & 1 & 1 & 1 & 1 & 1 & 1 & 1 & 1 & 1 & 1 & 1 & 1 & 1 & 1 & 1 & 1 \\
\hline
\end{tabular} 


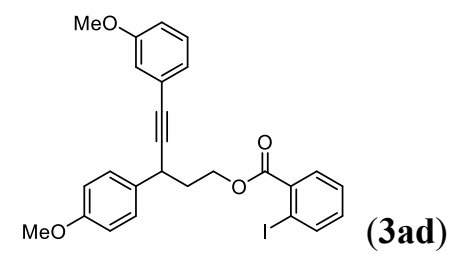

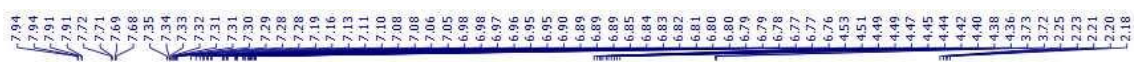

$300 \mathrm{MHz}$ in $\mathrm{CDCl}_{3}$
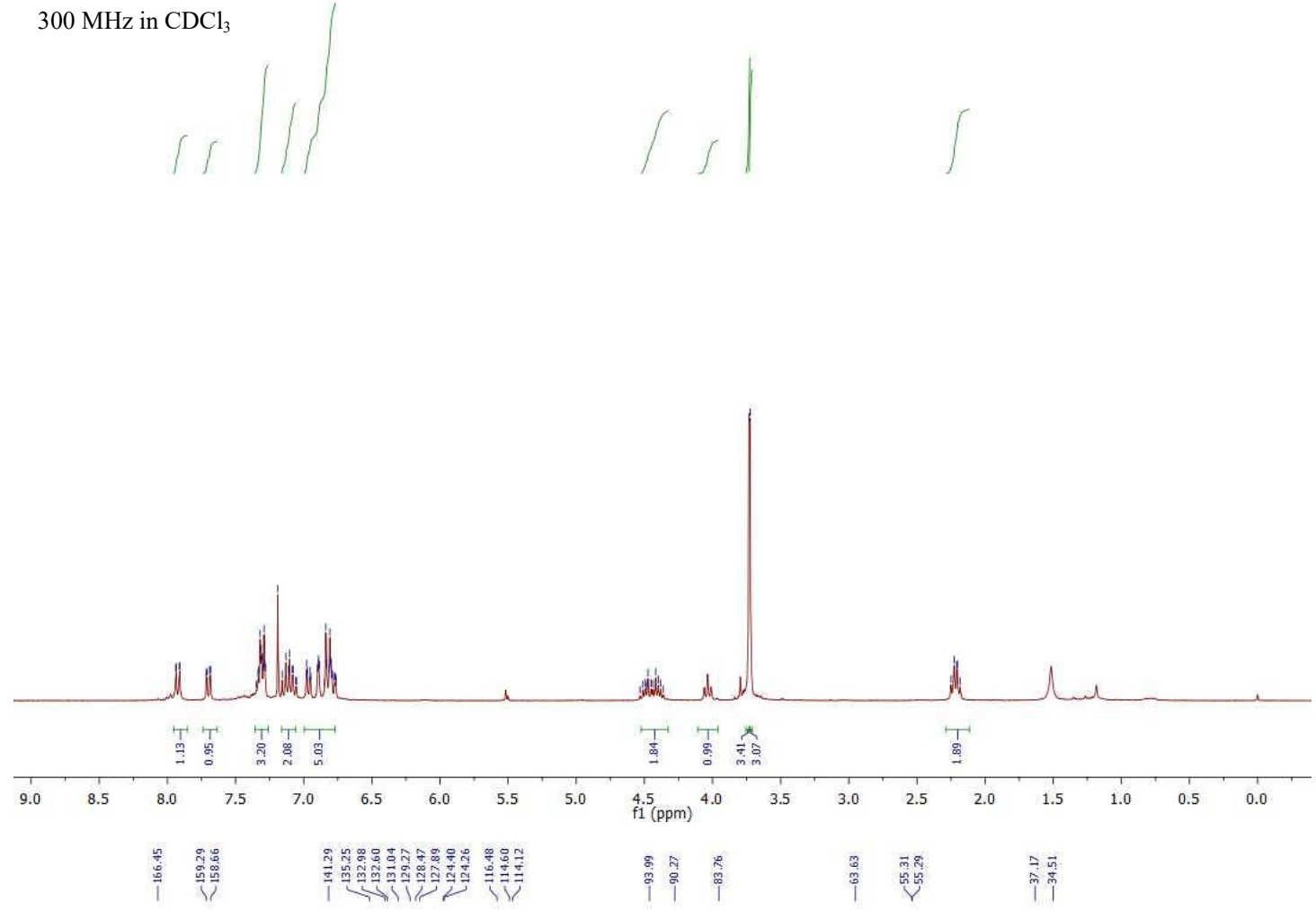

$151 \mathrm{MHz}$ in $\mathrm{CDCl}_{3}$

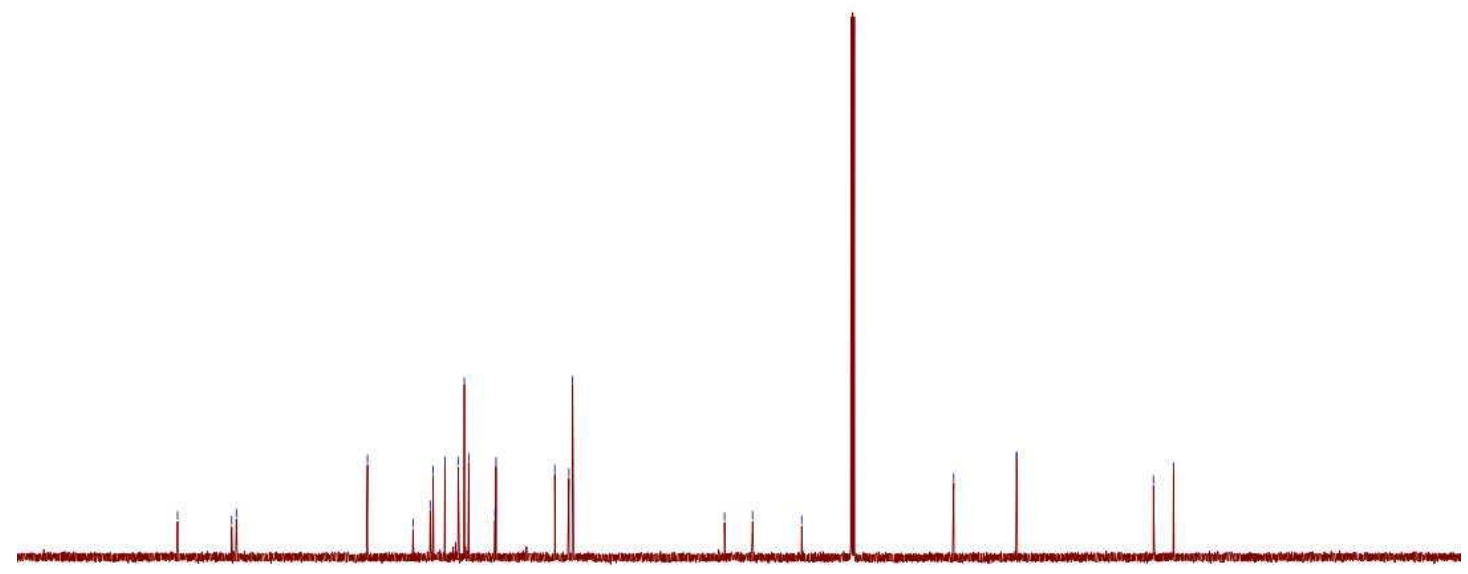

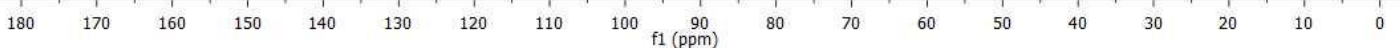




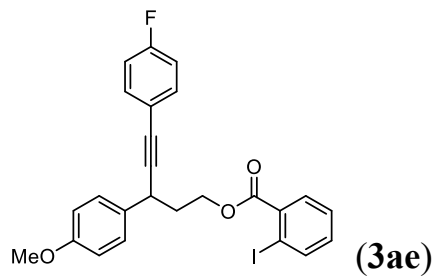

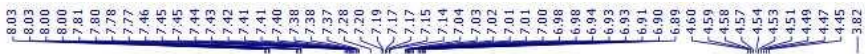

$300 \mathrm{MHz}$ in $\mathrm{CDCl}_{3}$
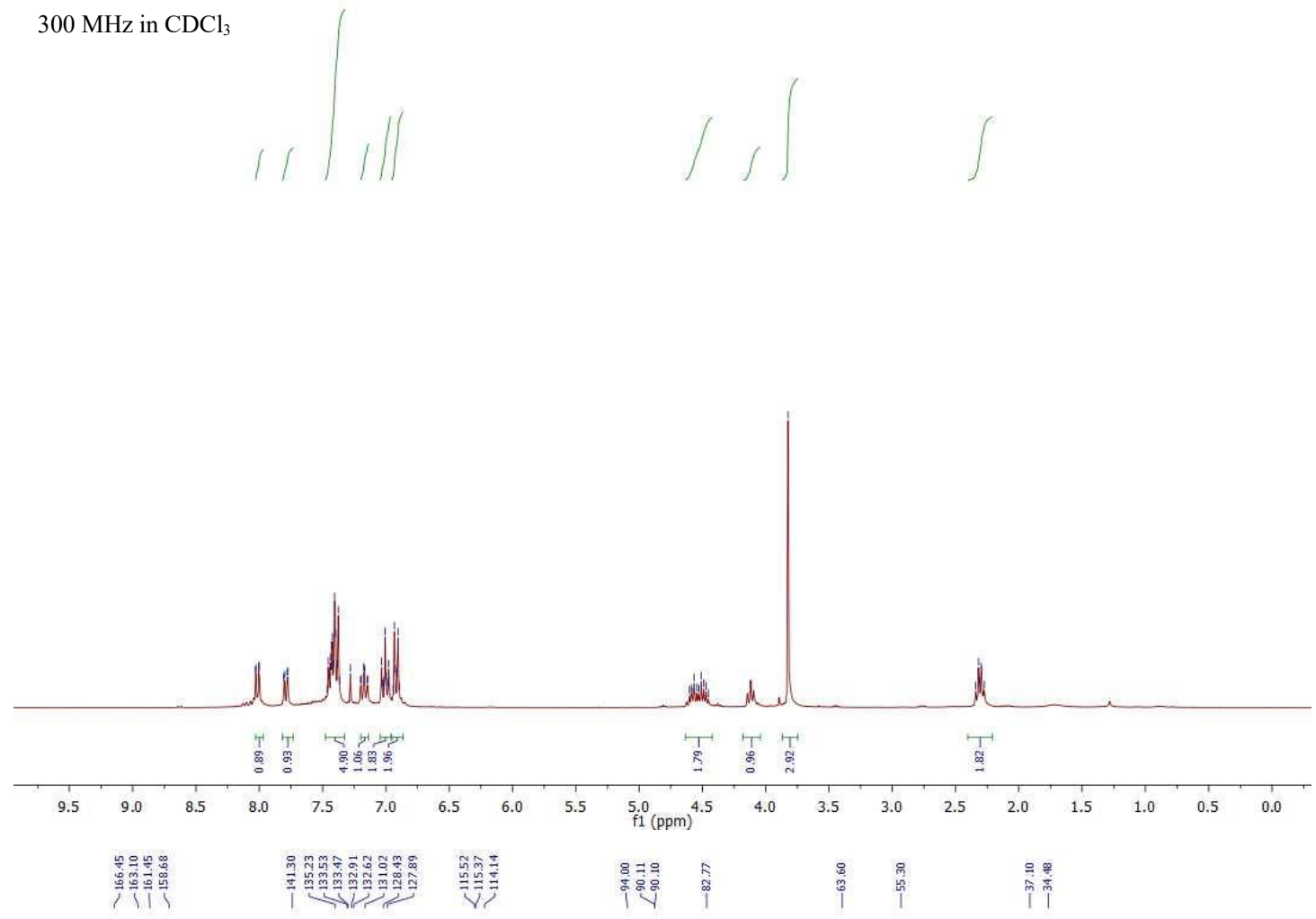

$151 \mathrm{MHz}$ in $\mathrm{CDCl}_{3}$
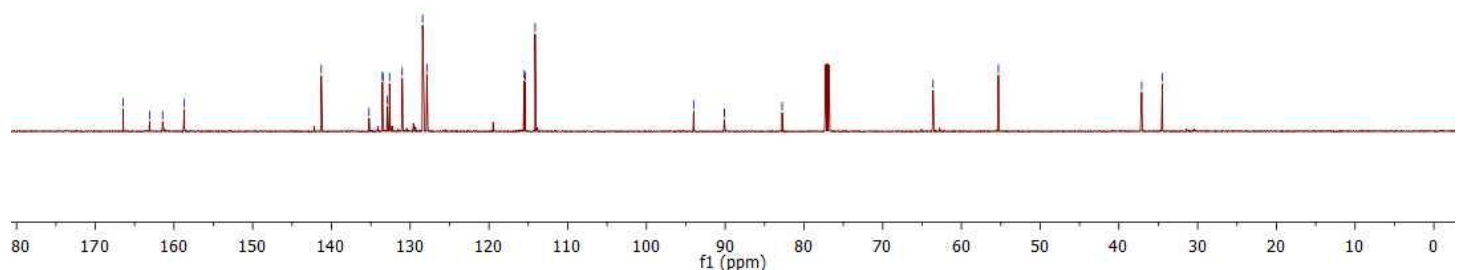
$\stackrel{\square}{\vec{i}}$

$282 \mathrm{MHz}$ in $\mathrm{CDCl}_{3}$

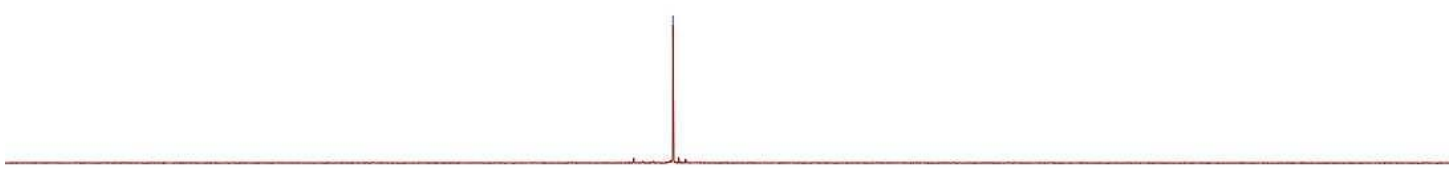

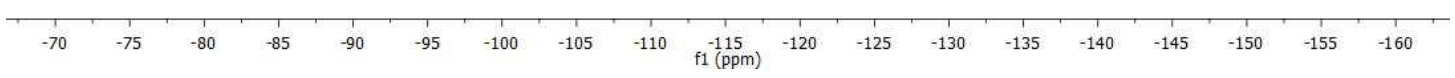

S63 


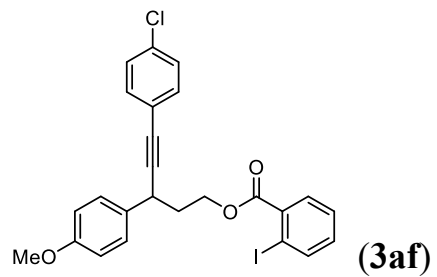

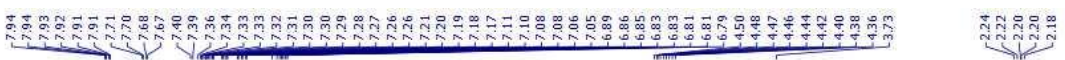

$300 \mathrm{MHz}$ in $\mathrm{CDCl}_{3}$
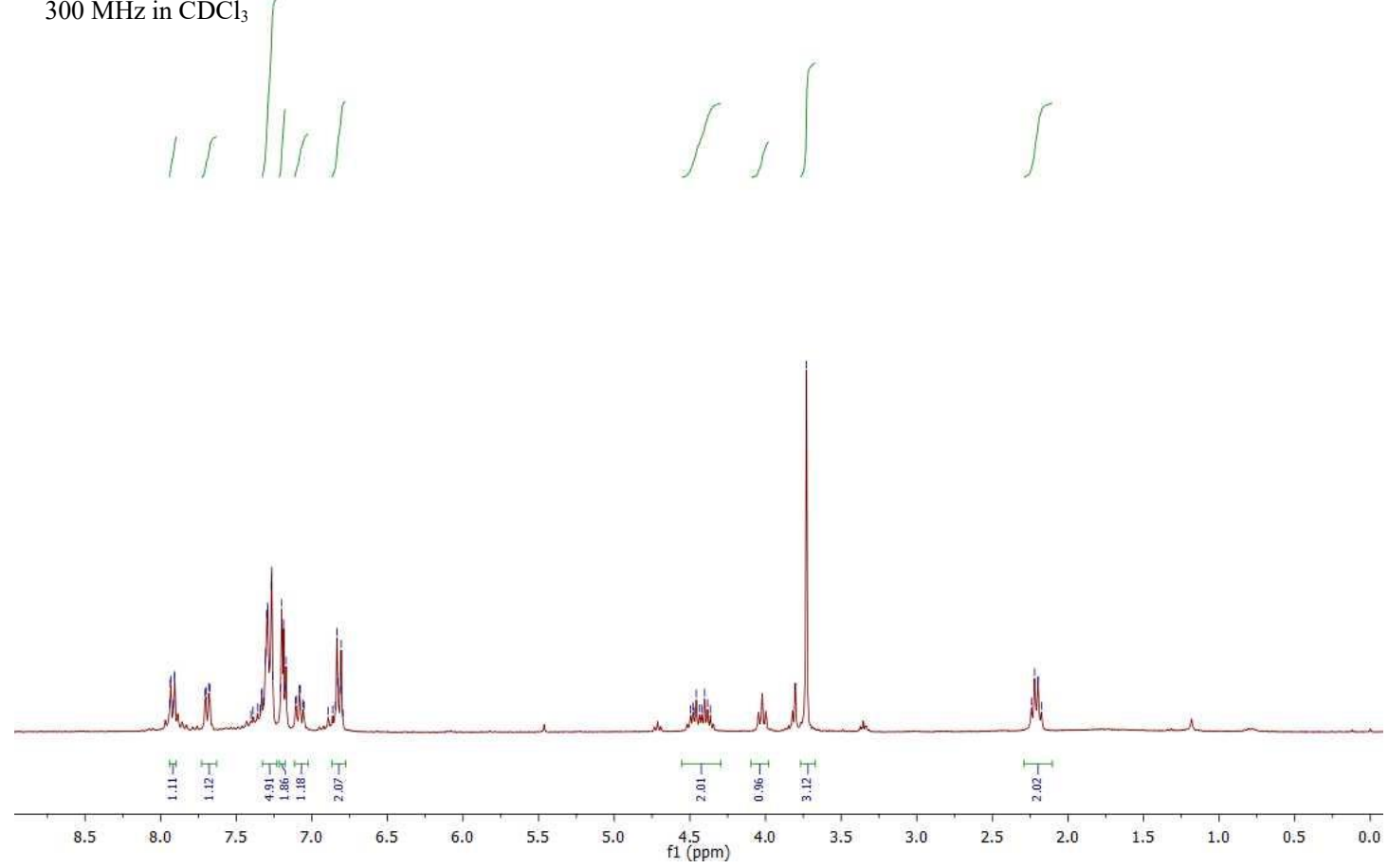

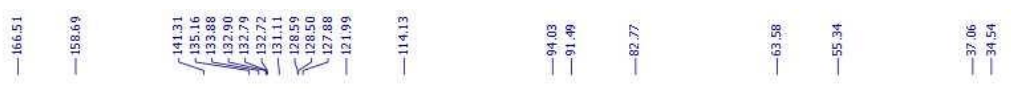

$76 \mathrm{MHz}$ in $\mathrm{CDCl}_{3}$

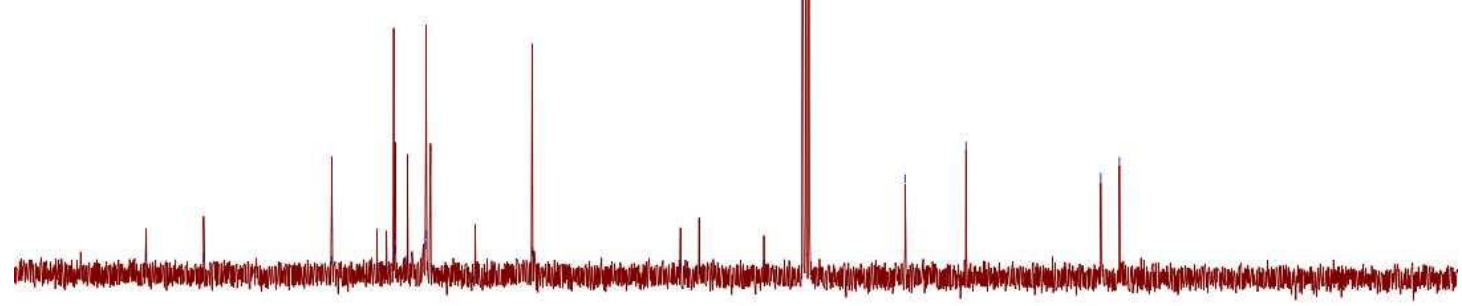

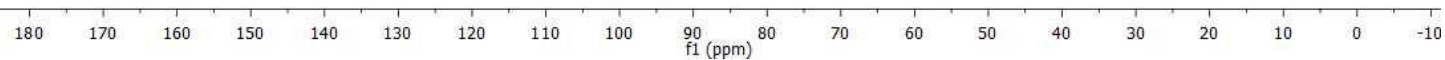


<smiles>COc1ccc(CCC(=O)c2ccccc2I)cc1</smiles>

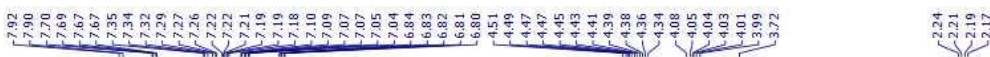

$300 \mathrm{MHz}$ in $\mathrm{CDCl}_{3}$
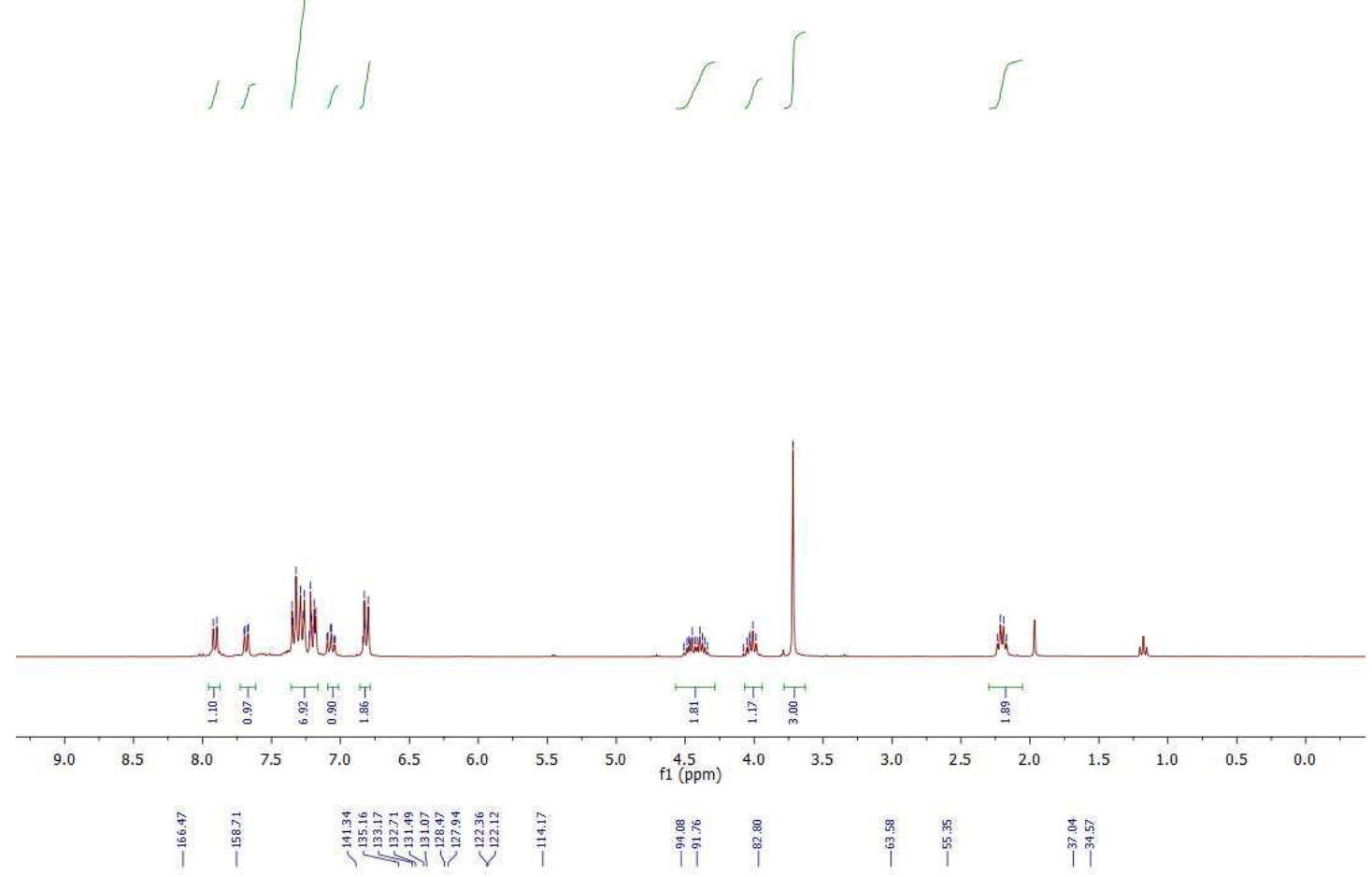

$76 \mathrm{MHz}$ in $\mathrm{CDCl}_{3}$

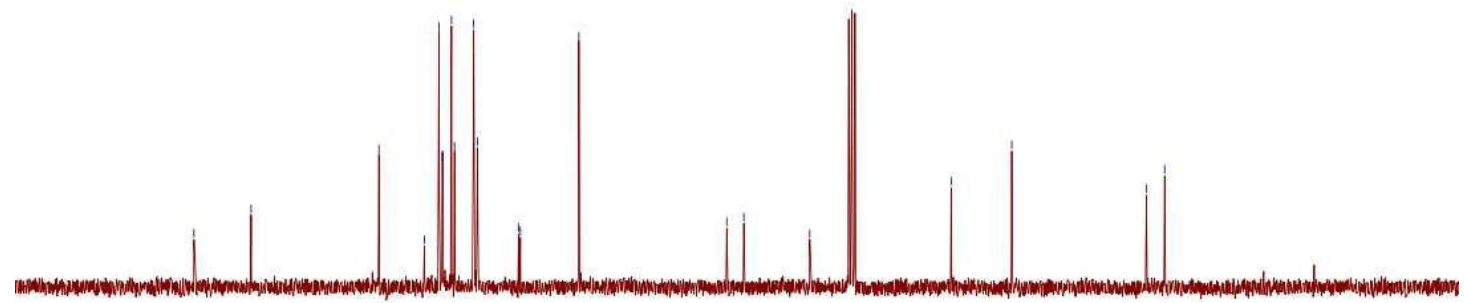

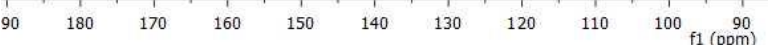




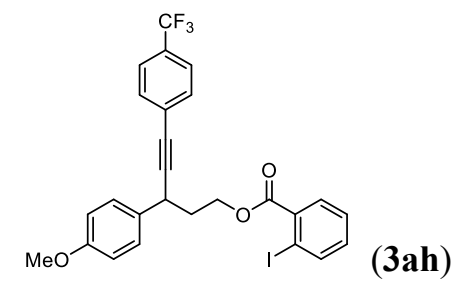

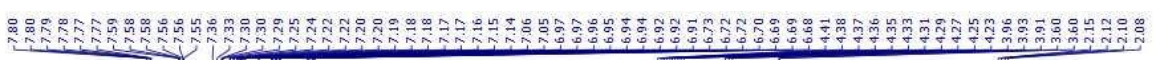

$300 \mathrm{MHz}$ in $\mathrm{CDCl}_{3}$

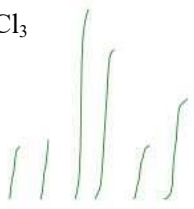

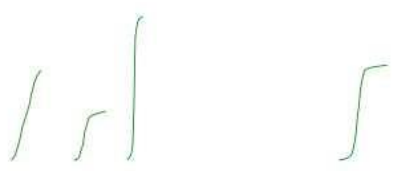

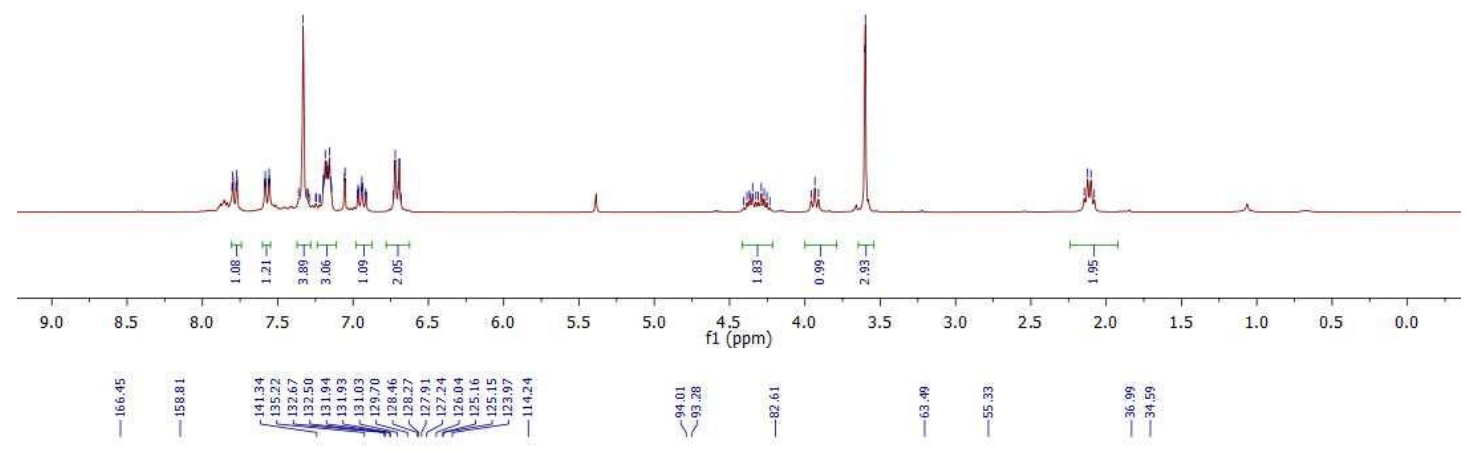

$126 \mathrm{MHz}$ in $\mathrm{CDCl}_{3}$

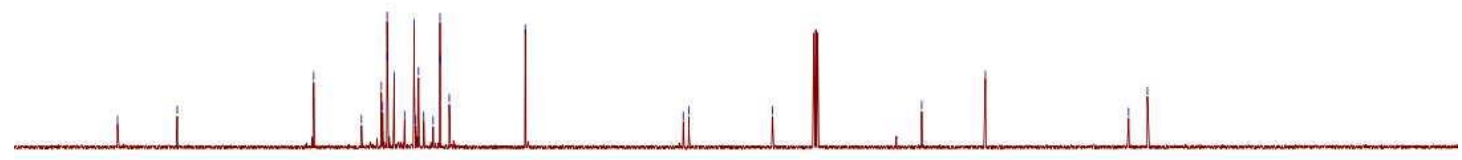

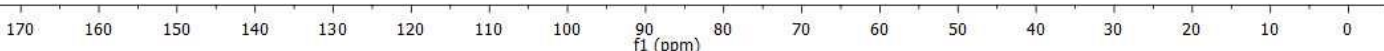


$282 \mathrm{MHz}$ in $\mathrm{CDCl}_{3}$

iั
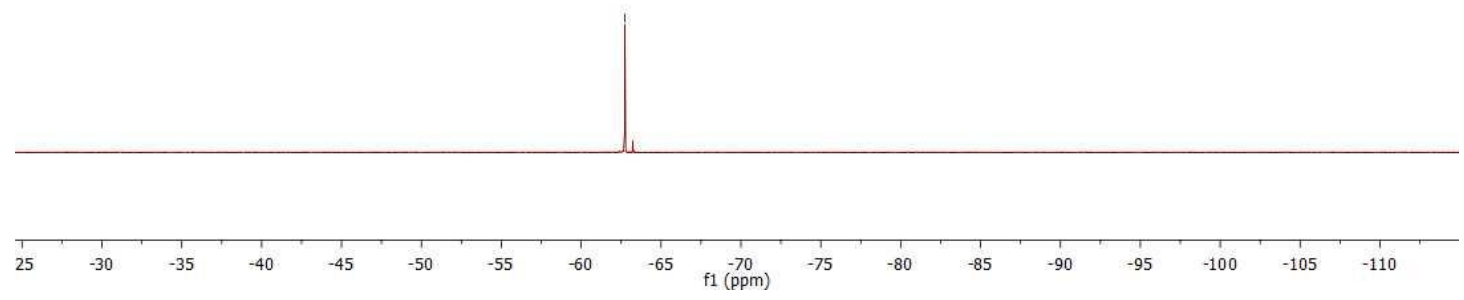


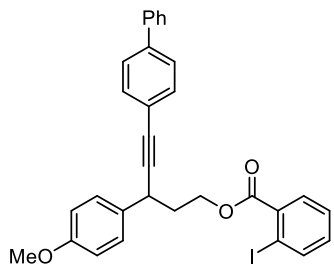

(3ai)

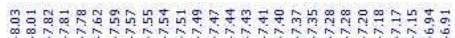

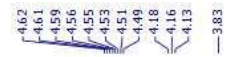

$300 \mathrm{MHz}$ in $\mathrm{CDCl}_{3}$
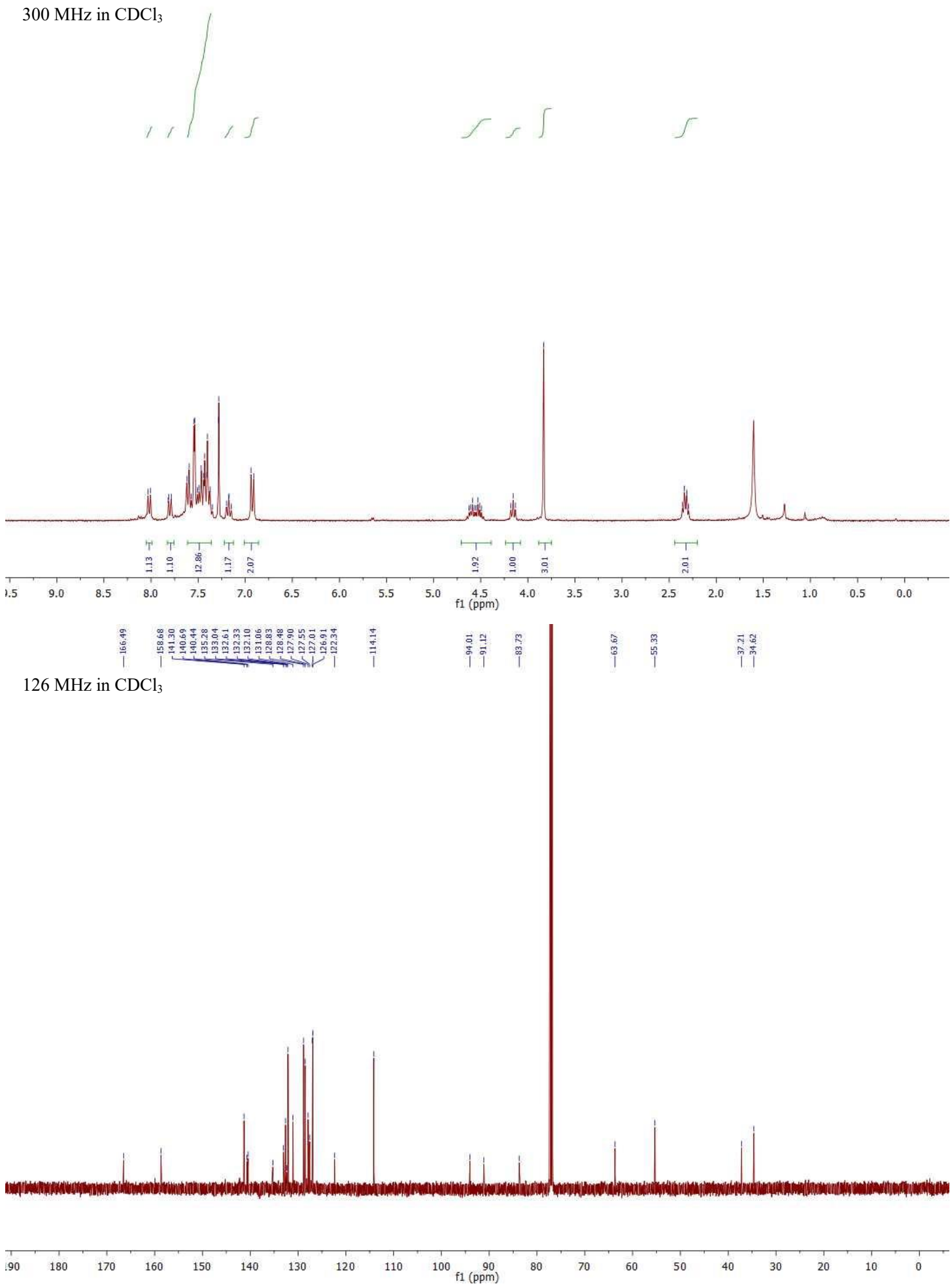

S68 
<smiles>Cc1ccc(C=C(CCOC(=O)c2ccccc2I)c2ccc(O)cc2)cc1</smiles>

(3aj)

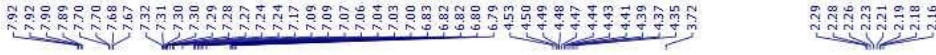

$300 \mathrm{MHz}$ in $\mathrm{CDCl}_{3}$
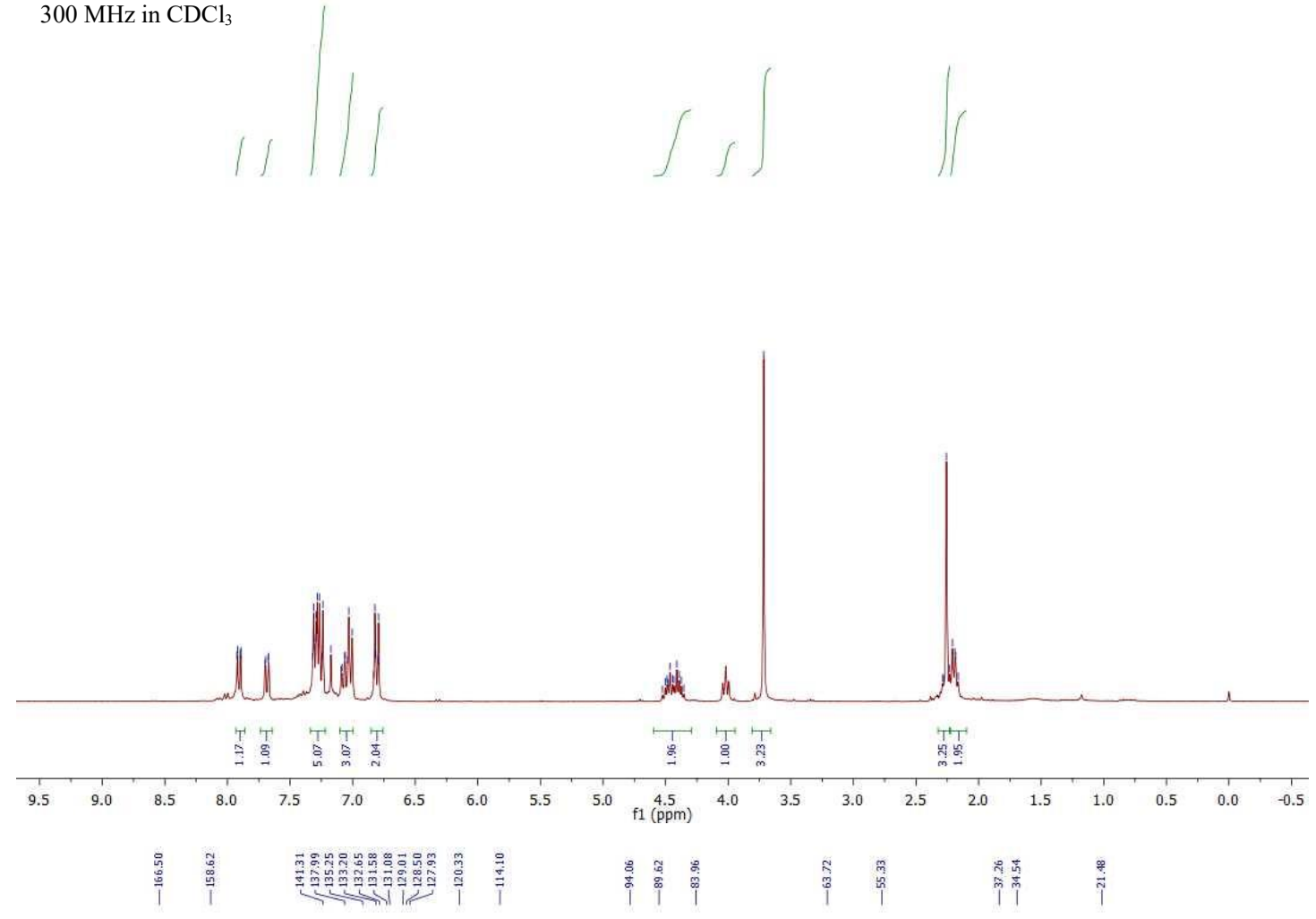

$76 \mathrm{MHz}$ in $\mathrm{CDCl}_{3}$
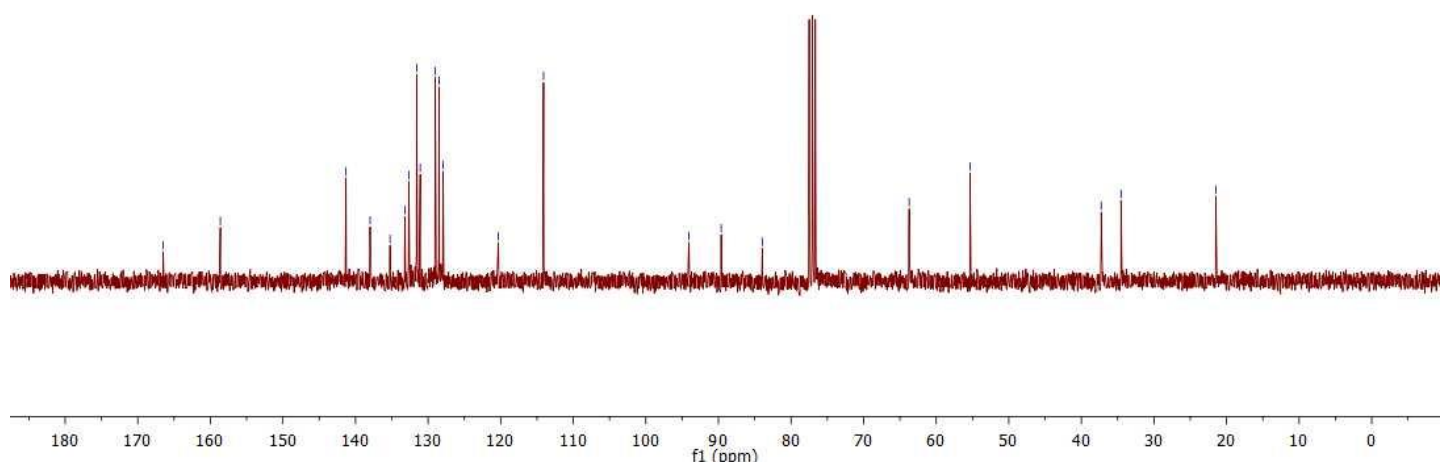


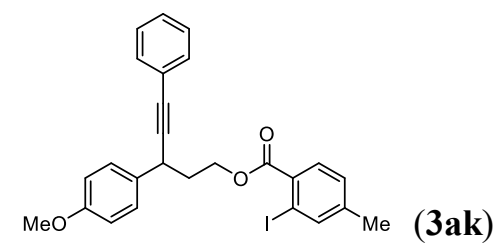

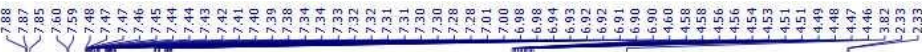

$300 \mathrm{MHz}$ in $\mathrm{CDCl}_{3}$
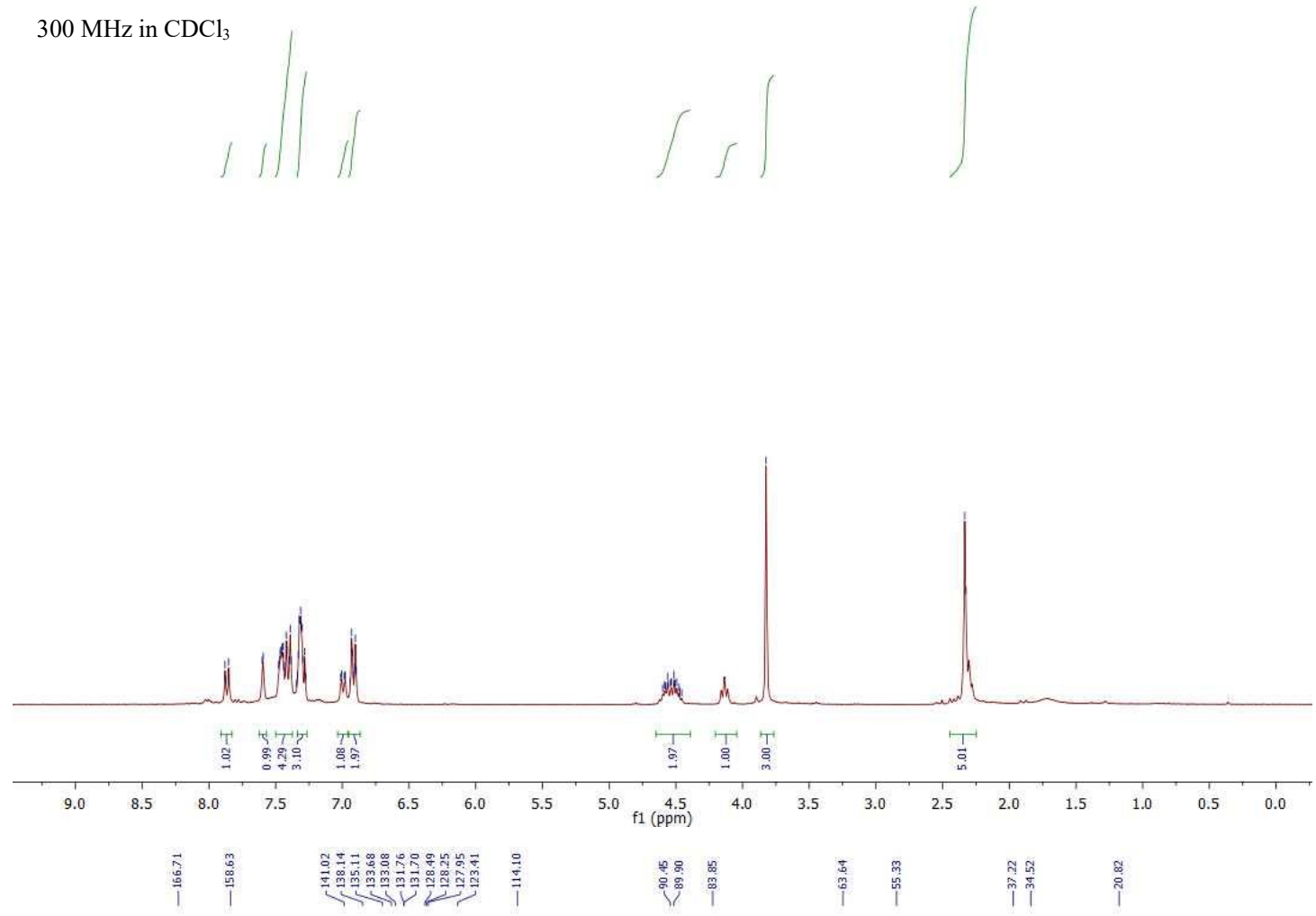

$76 \mathrm{MHz}$ in $\mathrm{CDCl}_{3}$

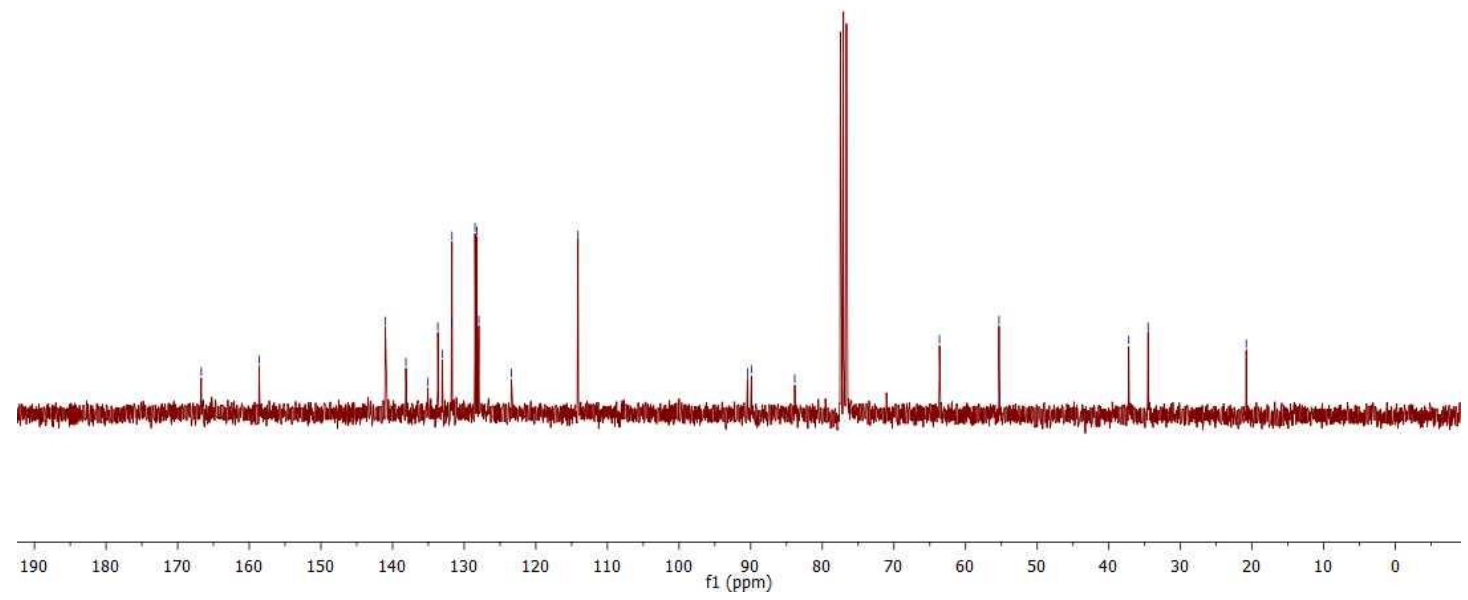




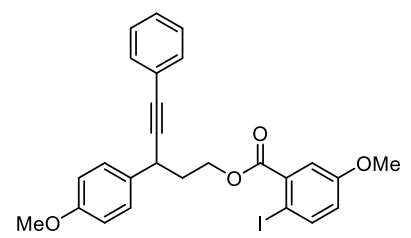

(3al)

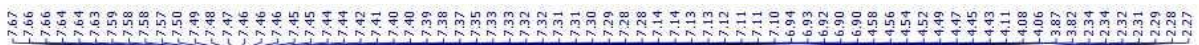

$300 \mathrm{MHz}$ in $\mathrm{CDCl}_{3}$
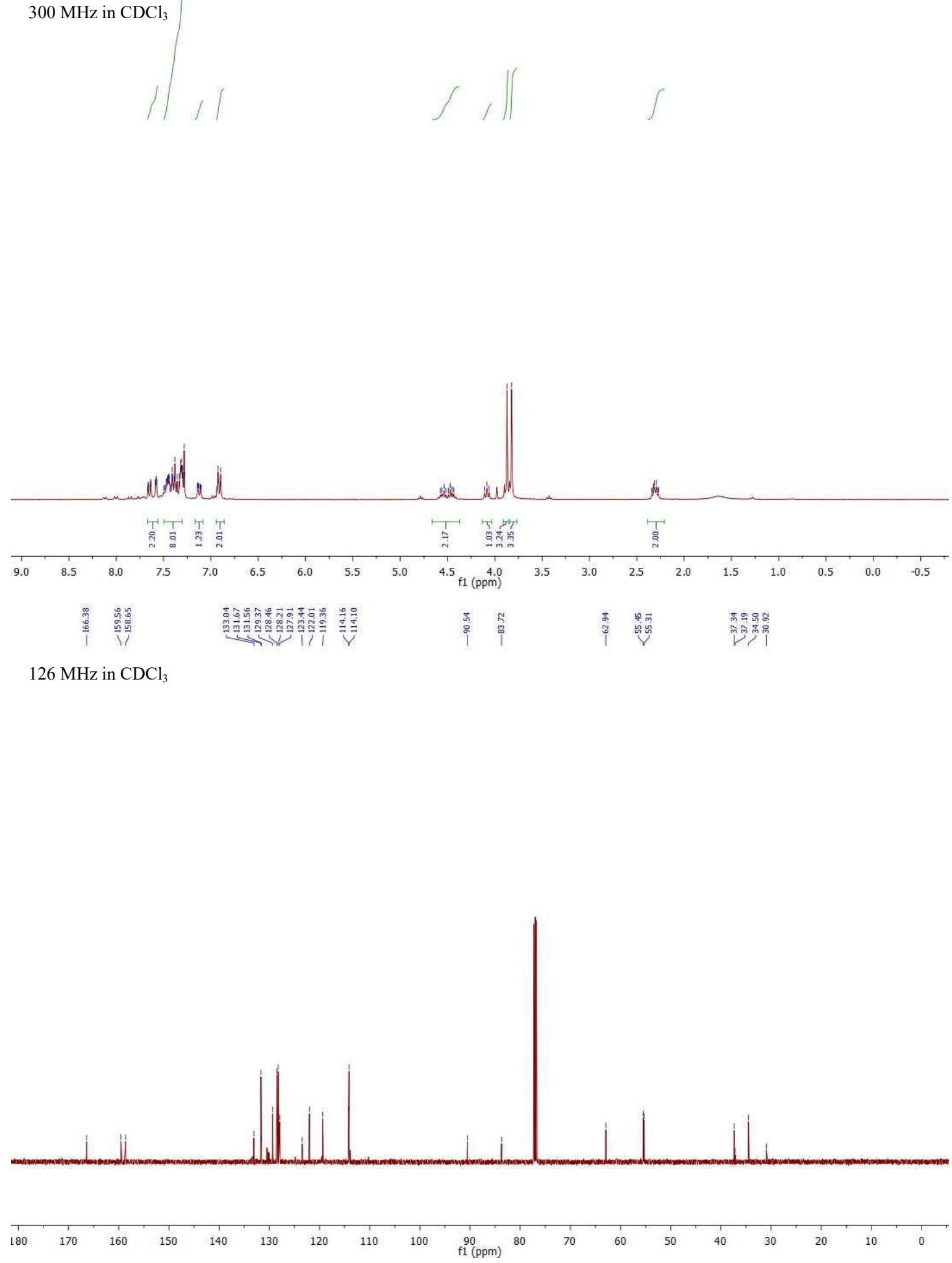

S71 


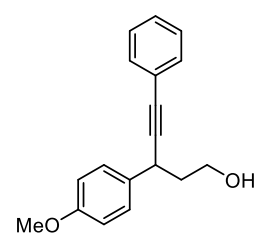

(4a)

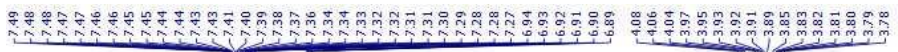

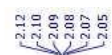
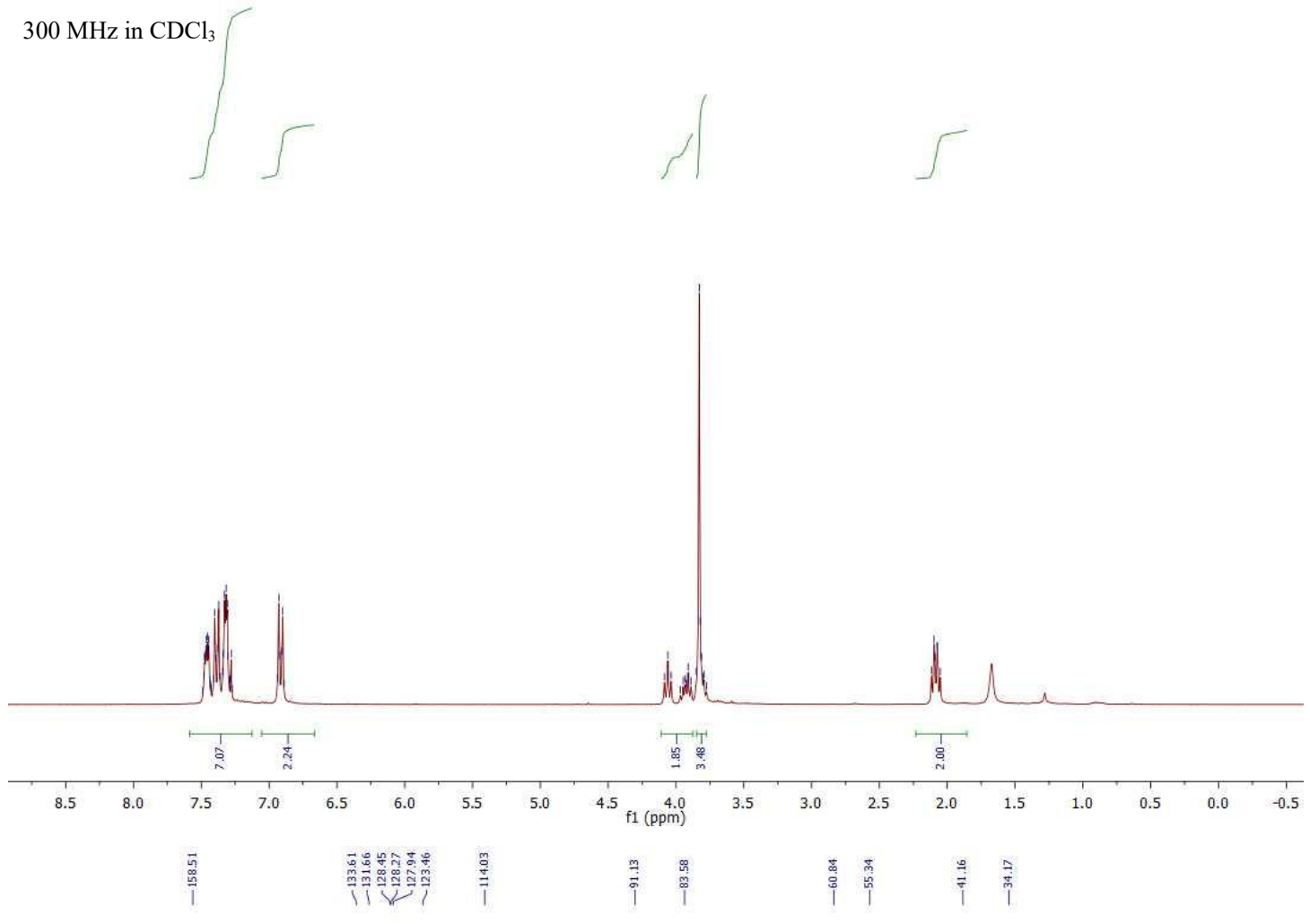

$76 \mathrm{MHz}$ in $\mathrm{CDCl}_{3}$
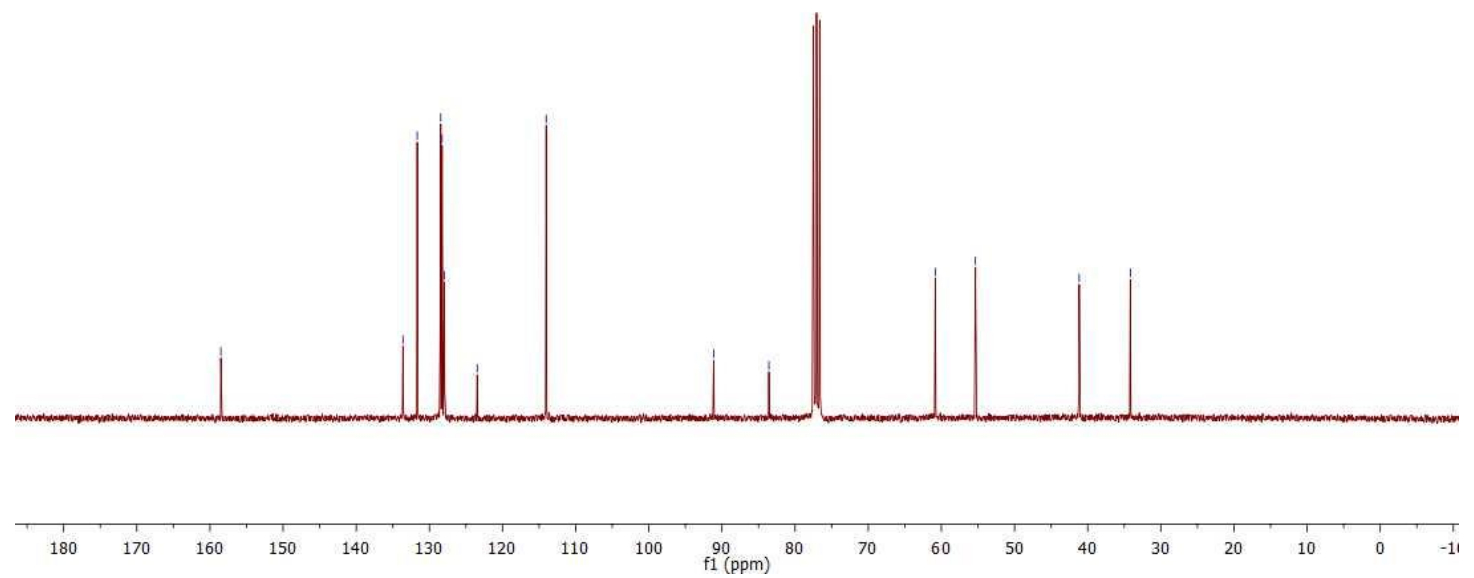

S72 


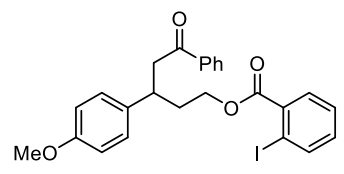

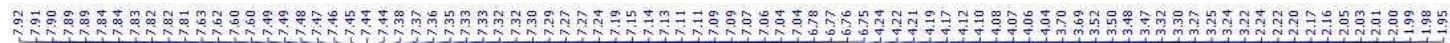
$300 \mathrm{MHz}$ in $\mathrm{CDCl}_{3}$
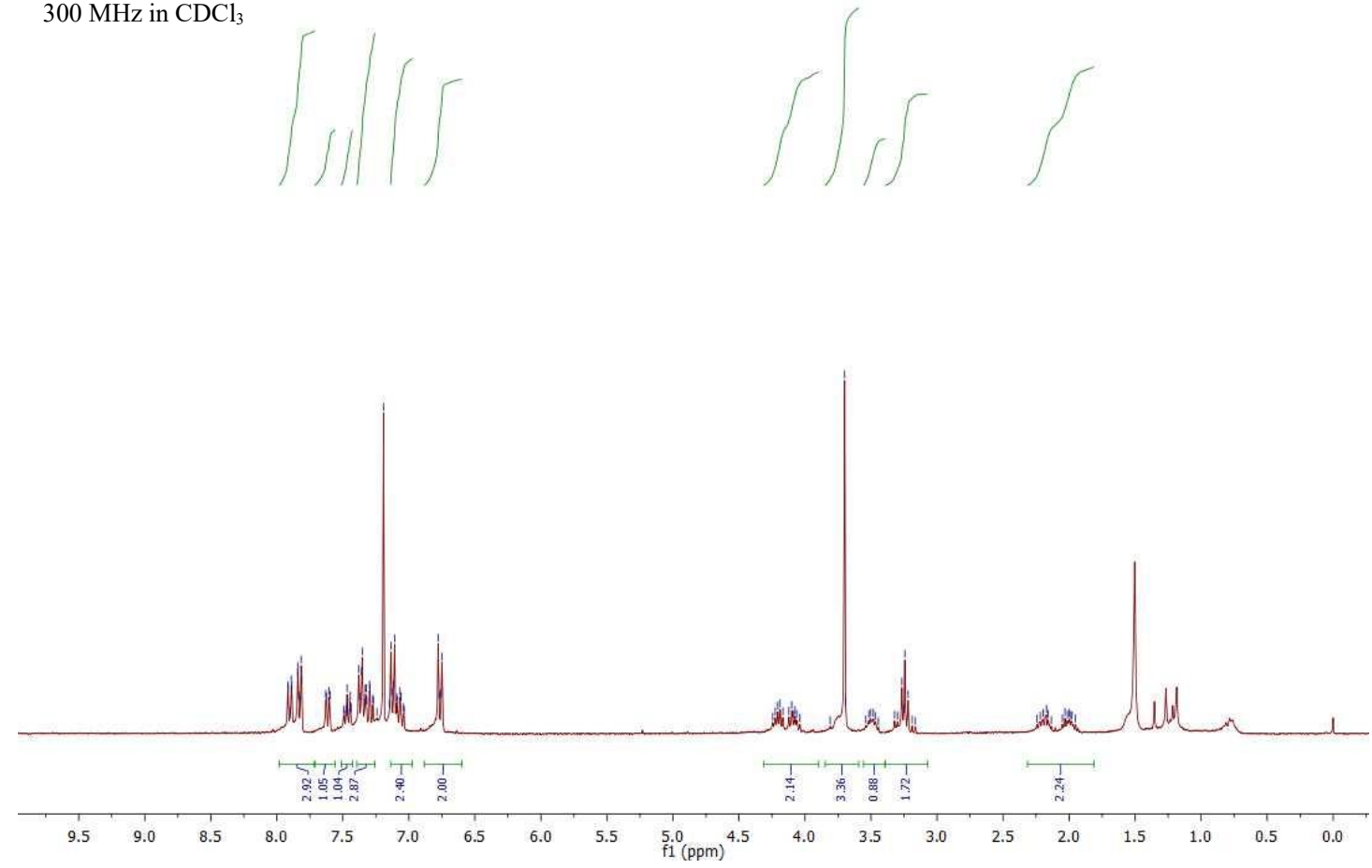

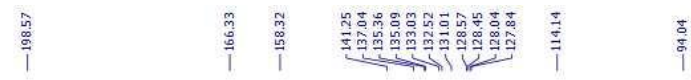

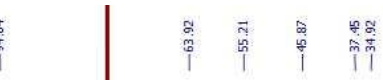

$151 \mathrm{MHz}$ in $\mathrm{CDCl}_{3}$

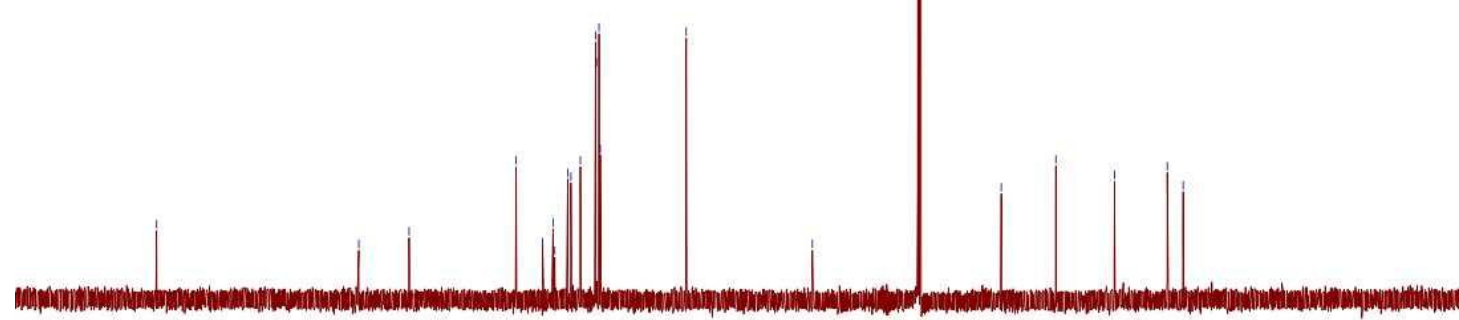

$\begin{array}{llllllllllllll}20 & 210 & 200 & 190 & 180 & 170 & 160 & 150 & 140 & 130 & 120 & \underset{f 1}{11}(\mathrm{ppm}) \\ \mathrm{f} & 100\end{array}$ 


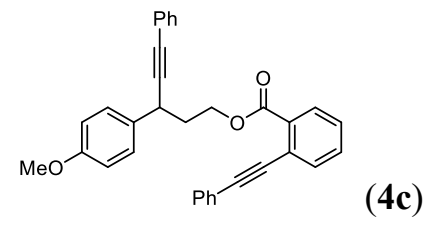

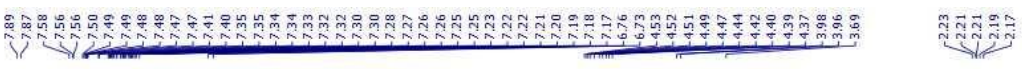

$300 \mathrm{MHz}$ in $\mathrm{CDCl}_{3}$

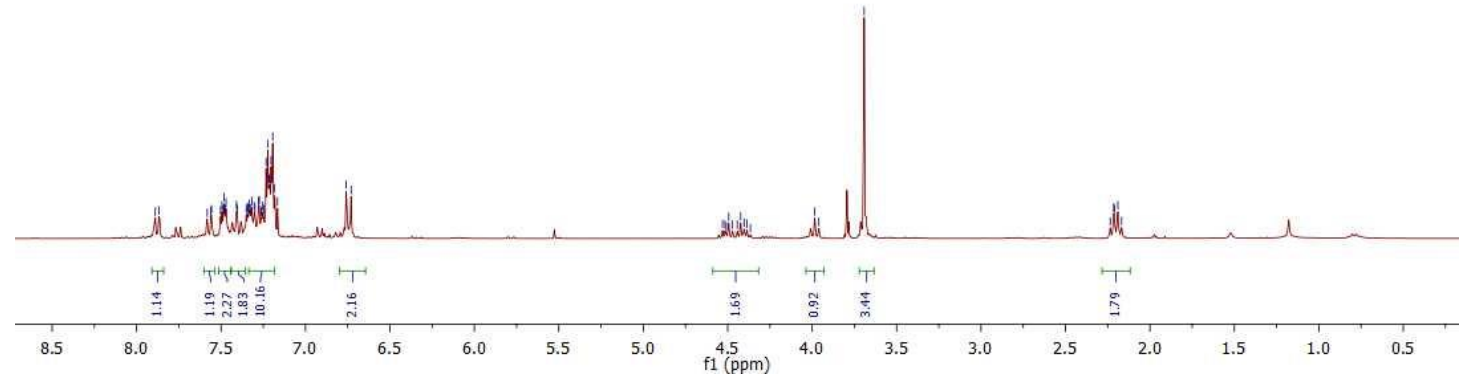

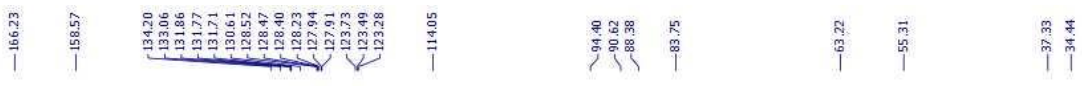

$76 \mathrm{MHz}$ in $\mathrm{CDCl}_{3}$

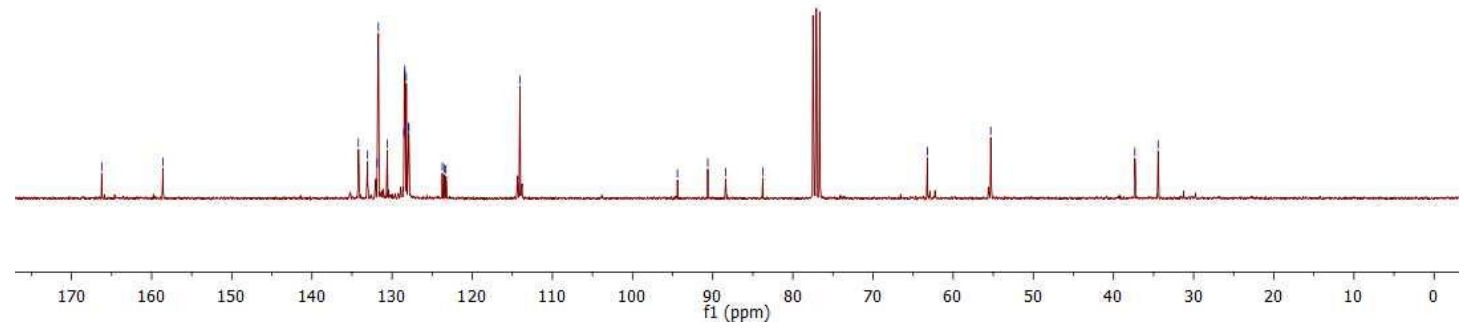




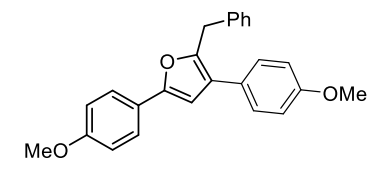

(4d)

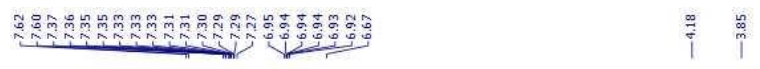

$300 \mathrm{MHz}$ in $\mathrm{CDCl}_{3}$

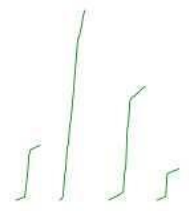

$\stackrel{\infty}{\stackrel{\infty}{\infty}} \stackrel{\infty}{i}$
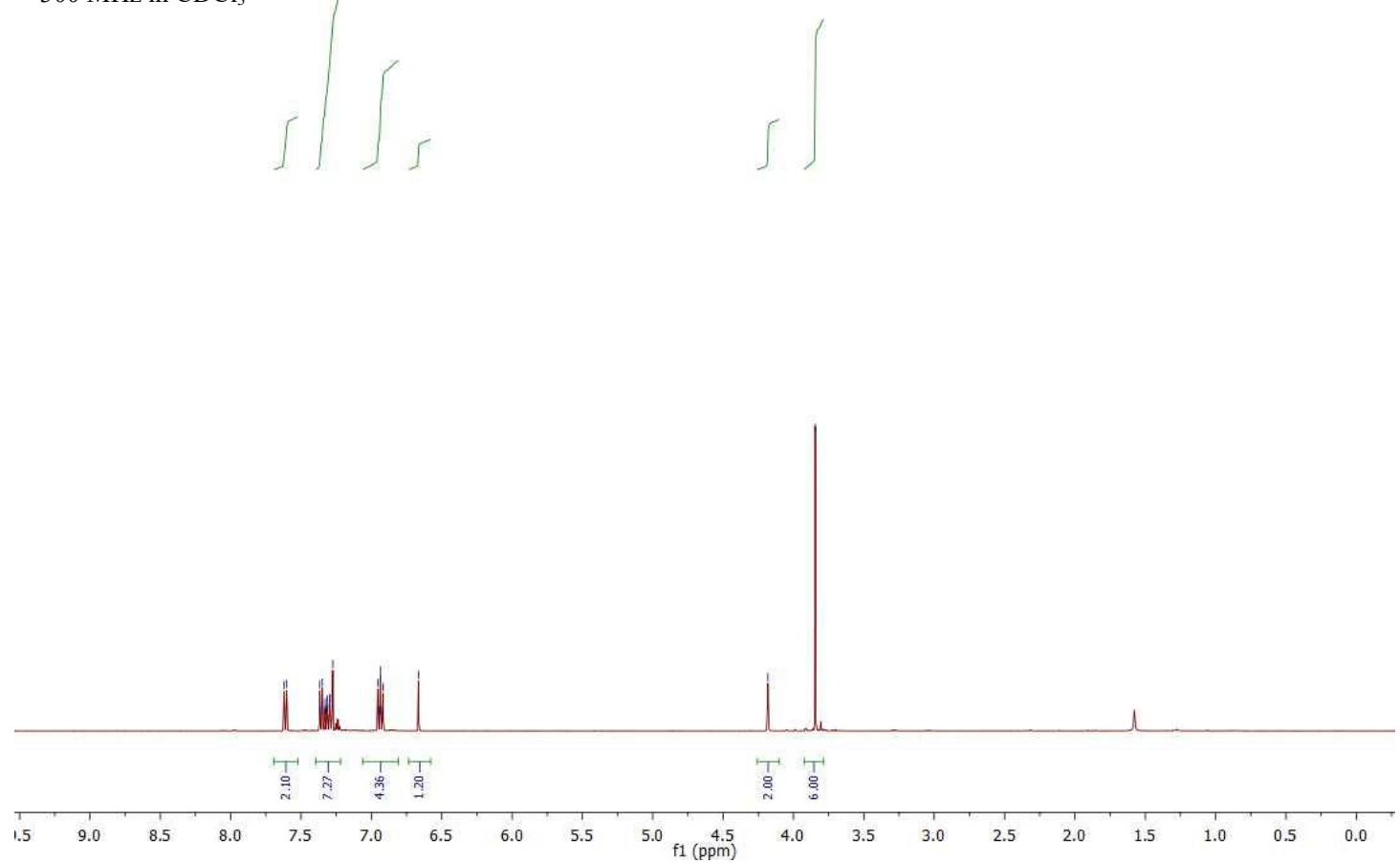

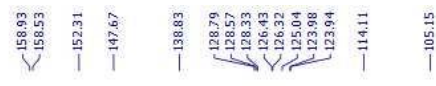

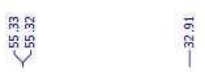

$126 \mathrm{MHz}$ in $\mathrm{CDCl}_{3}$

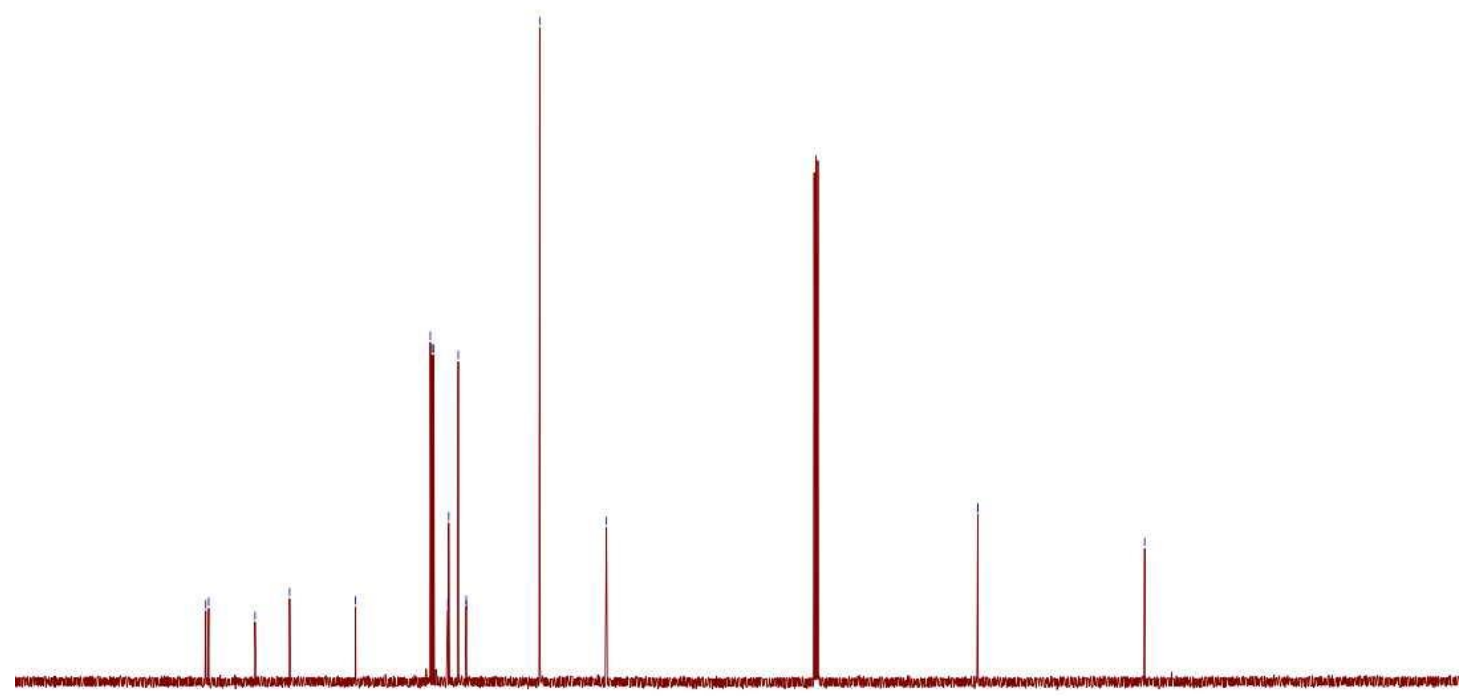

$\begin{array}{llllllllll}180 & 170 & 160 & 150 & 140 & 130 & 120 & 110 & 100 & 90 \\ \mathrm{f1}(\mathrm{ppm}) & 80\end{array}$

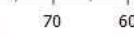

